\title{
Search for Physics Beyond the Standard Model Using Measurements of CP Violating Asymmetries in Rare B Decays: B0 to K0(S) Pi0 and B0 to K0(S) Pi0 Gamma
}

\author{
Dmytro Kovalskyi \\ Stanford Linear Accelerator Center \\ Stanford University \\ Stanford, CA 94309 \\ SLAC-Report-844 \\ Prepared for the Department of Energy \\ under contract number DE-AC02-76SF00515
}

Printed in the United States of America. Available from the National Technical Information Service, U.S. Department of Commerce, 5285 Port Royal Road, Springfield, VA 22161. 
This document, and the material and data contained therein, was developed under sponsorship of the United States Government. Neither the United States nor the Department of Energy, nor the Leland Stanford Junior University, nor their employees, nor their respective contractors, subcontractors, or their employees, makes an warranty, express or implied, or assumes any liability of responsibility for accuracy, completeness or usefulness of any information, apparatus, product or process disclosed, or represents that its use will not infringe privately owned rights. Mention of any product, its manufacturer, or suppliers shall not, nor is it intended to, imply approval, disapproval, or fitness of any particular use. A royalty-free, nonexclusive right to use and disseminate same of whatsoever, is expressly reserved to the United States and the University. 


\title{
ABSTRACT
}

Title of dissertation:

\begin{abstract}
SEARCH FOR PHYSICS BEYOND THE STANDARD MODEL USING MEASUREMENTS OF $C P$ VIOLATING ASYMMETRIES IN RARE $B$ DECAYS: $B^{0} \rightarrow K_{S}^{0} \pi^{0}$ and $B^{0} \rightarrow K_{S}^{0} \pi^{0} \gamma$.
\end{abstract}

\section{Dmytro Kovalskyi, Doctor of Philosophy, 2005}

\section{Dissertation directed by: Professor Abolhassan Jawahery Department of Physics}

This dissertation presents measurements of time-dependent $C P$ violating asymmetries in the decays $B^{0} \rightarrow K_{S}^{0} \pi^{0}$ and $B^{0} \rightarrow K_{S}^{0} \pi^{0} \gamma$ based on RUN 1-4 data collected with the BABAR detector at the $\Upsilon(4 S)$ resonance operating at the PEP-II asymmetric $e^{+} e^{-}$collider at SLAC. It was found that the $C P$ violating asymmetry parameters are $S_{K_{S} \pi^{0}}=0.35_{-0.33}^{+0.30}$ (stat) \pm 0.04 (syst), $C_{K_{S} \pi^{0}}=0.06 \pm 0.18$ (stat) \pm 0.03 (syst), $S_{K^{* 0} \gamma}=-0.21 \pm 0.40$ (stat) \pm 0.05 (syst), $C_{K^{* 0} \gamma}=-0.40 \pm 0.23$ (stat) \pm 0.03 (syst), $S_{K_{S} \pi^{0} \gamma}=0.9 \pm 1.0$ (stat) \pm 0.2 (syst) and $C_{K_{S} \pi^{0} \gamma}=-1.0 \pm 0.5$ (stat) \pm 0.2 (syst), where $B^{0} \rightarrow K^{* 0} \gamma$ decays correspond to the $K_{S} \pi^{0}$ invariant mass interval of $[0.8,1.0] \mathrm{GeV}$ and $B^{0} \rightarrow K_{S}^{0} \pi^{0} \gamma$ of $[1.1,1.8] \mathrm{GeV}$. All results are consistent with the Standard Model predictions. 


\title{
SEARCH FOR PHYSICS BEYOND THE STANDARD MODEL USING MEASUREMENTS OF $C P$ VIOLATING ASYMMETRIES IN RARE $B$ DECAYS: $B^{0} \rightarrow K_{S}^{0} \pi^{0}$ and $B^{0} \rightarrow K_{S}^{0} \pi^{0} \gamma$.
}

by

\author{
Dmytro Kovalskyi
}

Dissertation submitted to the Faculty of the Graduate School of the University of Maryland, College Park in partial fulfillment of the requirements for the degree of

Doctor of Philosophy 2005

Advisory Committee:

Professor Abolhassan Jawahery, Chair/Advisor

Professor Robert W. Gammon

Professor Douglas Roberts

Professor Rabindra Mohapatra

Professor Nicholas Hadley 
(c) Copyright by

\section{Dmytro Kovalskyi}

2005 


\section{DEDICATION}

This dissertation is dedicated to my wife, my mother and father. Their sacrifices, support and love made this work possible. 


\section{ACKNOWLEDGMENTS}

I was fortunate enough to be a part of the Maryland group in BABAR experiment. Thanks to the wisdom and knowledge of its leader and my scientific adviser Hassan Jawahery, the group has always had the best people and played a key role in the experiment. Being a member of such a big collaboration as $B A B A R$, I had a chance to see how much the strong leadership means to the success of any endeavor.

I'm grateful to all support and help that I've got from two outstanding members

of the Maryland group - Amir Farbin and Wouter Hulsbergen. Amir provided me valuable advice and encouragement since I joined the group. He also helped me with the cultural adaptation to the new country. Many times I realized how right he was about different aspects of physics and life.

I would also like to thank Wouter Hulsbergen for all the attention he paid to my numerous questions. His deep understanding and critical thinking made a huge impact on the progress of my studies. Working with him I witnessed how efficient and productive one person can be, which also stimulated my own development.

Quite often when I needed a confirmation of my understanding of some subtle question or I couldn't find anyone to explain to me some physics effect, I found myself looking for Bill Dunwoodie. I want to thank him for always being considerate. 
His feedback on many fine questions in high energy physics was indispensable.

I would like to thank Yuval Grossman for finding time and desire to explain to me many aspects of the modern theoretical physics and different phenomenological models. I'm also grateful to many other theorists that broaden my view of physics and in particular the Theoretical Physics Group at SLAC.

It was a life changing experience to work in such a big collaboration in one of the best laboratories of high energy physics in the world. I'm thankful to many people in $B A B A R$ and SLAC for an unforgettable experience working with them. 


\section{Contents}

1 Introduction 1

2 Theoretical aspects of the measurements 4

2.1 The Standard Model . . . . . . . . . . . . . . . . . . . 4

$2.2 C P$ violation . . . . . . . . . . . . . . . . . . . . 7

2.3 Physics beyond the Standard Model . . . . . . . . . . . . . . . . . 12

2.4 Phenomenology of $B$ meson decays . . . . . . . . . . . . . . . . . 15

2.5 Experimental observables . . . . . . . . . . . . . . . . 19

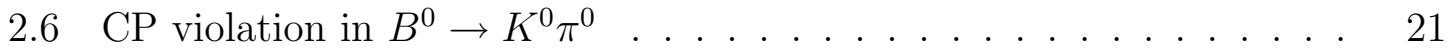

2.6.1 Estimation of the sub-leading amplitude effect on the expected $C P$ asymmetry using $\mathrm{SU}(3)$ approximation . . . . . . . . 23

2.6.2 Estimation of the sub-leading amplitude effect on the expected CP asymmetry using the QCD factorization approach . . . . 28

2.7 CP violation in $B^{0} \rightarrow K_{S}^{0} \pi^{0} \gamma \ldots \ldots$. . . . . . . . . . . . . . 29

2.7.1 Hadronic corrections . . . . . . . . . . . . . . . . . . . 31 
2.7.2 Alternative methods of measuring the photon polarization . . 34

2.8 Summary . . . . . . . . . . . . . . . . . 36

3 The BABAR Detector $\quad 38$

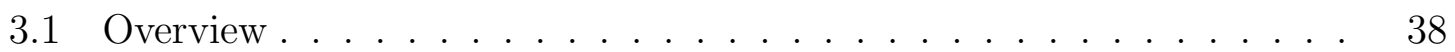

3.2 The collider and beam parameters . . . . . . . . . . . . . . . . 42

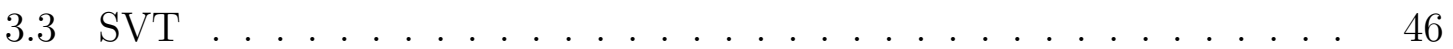

3.4 The Drift Chamber . . . . . . . . . . . . . . . . . . . . 49

3.5 The EMC . . . . . . . . . . . . . . . . . . 51

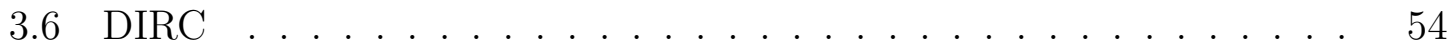

$3.7 \quad \mathrm{IFR} \ldots \ldots \ldots \ldots \ldots \ldots \ldots$

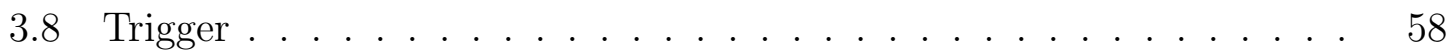

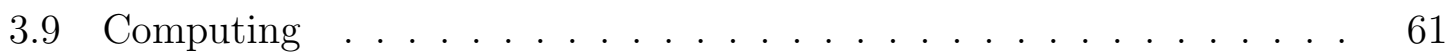

3.10 Monte Carlo simulations . . . . . . . . . . . . . . . . 63

4 Experimental aspects of the physics analyses $\quad 66$

4.1 Measurement of the time-dependent $C P$ asymmetries . . . . . . . . . 66

4.1.1 B meson flavor tagging . . . . . . . . . . . . . . . 68

4.1.2 Reconstruction of the $B$ meson decay vertices for partially reconstructed decays . . . . . . . . . . . . . . . 72

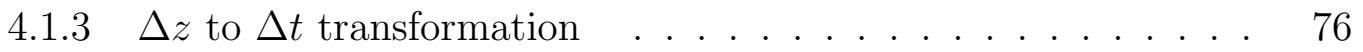

4.1.4 Resolution model . . . . . . . . . . . . . . . . . . . . . 78 
4.1.5 The beam constrained vertex reconstruction . . . . . . . . 79

4.2 Continuum background suppression . . . . . . . . . . . 83

4.3 Kinematic variables for the $B$ meson selection . . . . . . . . . . . . 88

4.3.1 Maximum likelihood fit . . . . . . . . . . . . . . 94

$5 \quad B^{0} \rightarrow K_{S}^{0} \pi^{0}$ analysis $\quad 97$

5.1 Candidate selection . . . . . . . . . . . . . . . . . . . . . 97

5.2 Maximum likelihood fit . . . . . . . . . . . . . 100

$5.3 B \bar{B}$ background . . . . . . . . . . . . . . . . . . . . 102

5.4 Results . . . . . . . . . . . . . . . . . 103

5.5 Validations and systematic effects evaluation . . . . . . . . . . . 104

5.6 Summary . . . . . . . . . . . . . . . . . . . 107

$6 \quad B^{0} \rightarrow K_{S}^{0} \pi^{0} \gamma$ analysis $\quad 110$

6.1 The effect of resonances on the $C P$ violating observables . . . . . . . 111

6.2 Candidate selection . . . . . . . . . . . . . . . . 114

$6.3 B \bar{B}$ background . . . . . . . . . . . . . . . . . . 119

6.4 The maximum likelihood fit . . . . . . . . . . . . . . . . . 121

6.5 Results . . . . . . . . . . . . . . . . . . . 124

6.6 Validations and systematic effects evaluation . . . . . . . . . . 128

6.7 Summary . . . . . . . . . . . . . . . . . 130

7 Conclusions $\quad 132$ 


\section{Chapter 1}

\section{Introduction}

Progress in high energy physics:

particles discovered yesterday are

used for calibration today, and will

be the background tomorrow.

The Standard Model of the elementary particle interactions successfully describes all phenomena observed so far at high energy particle colliders with the center of mass energy up to few $\mathrm{TeV}$. Despite this remarkable achievement, the Standard Model requires a high degree of fine tuning of the model parameters as soon as the energy scale is increased just by one or two orders of magnitude. Astrophysical observations also suggest that a major part of matter and energy in the Universe is still unknown. All this makes us believe that there are new physics phenomena, which can be found at the currently operating high energy facilities or the next generation colliders such 
as LHC or ILC.

While higher energy colliders, which can directly probe the new energy scale, are being designed and built, new physics effects can be probed indirectly in high precision measurements at lower energies. Such measurements can be sensitive to new physics effects at much higher energy scale. One of the possible ways to achieve this goal is to study $C P$ violating effects in rare $B$-meson decays, which are dominated by loop diagrams.

$C P$ violation is one of those rare phenomena in nature that leaves anyone surprised when it is discovered. Most physicists believed that $C P$ symmetry is an exact symmetry of nature until the experiment by Christenson, Cronin, Fitch and Turlay in 1964 proved that $C P$ symmetry can be violated. A surprising aspect of the observation was that $C P$ is violated only at the level of a few parts per thousand. Soon after that Andrei Saharov pointed out that $C P$ violation is one of the conditions required to explain the apparent asymmetry of matter and anti-matter in the Universe.

For more than three decades studies of $C P$ violation were limited to the neutral kaons. In the year 2000 a new era in $C P$ violation studies began with the observation of $C P$ violation in the neutral $B$ meson by the BABAR and the Belle experiments operating at the SLAC and the KEK B factory accelerators. Precision measurements of $C P$ violating asymmetries and the Kobayashi-Maskawa quark mixing matrix provide a solid basis for new physics phenomena searches. Even though there is much to be done to measure all parameters of the Standard Model with high accuracy, it 
is already possible to use $C P$ violation as a probe of new phenomena.

This dissertation presents measurements of the $C P$ violating asymmetries in $B$ meson decays, $B^{0} \rightarrow K_{S}^{0} \pi^{0}$ and $B^{0} \rightarrow K_{S}^{0} \pi^{0} \gamma$, which represent a case of the gluonic and the electro-magnetic penguin $b \rightarrow s$ decays. These measurements required the development of a new vertex reconstruction technique to measure the decay time of B mesons in final states where no charged particles are present at the B decay vertex. Most studies of the sensitivity of the B-factories to new physics phenomena include both decay modes among a few of the most sensitive and theoretically clean ways to probe for physics beyond the Standard Model. 


\section{Chapter 2}

\section{Theoretical aspects of the}

\section{measurements}

\subsection{The Standard Model}

The Standard Model is based on the electroweak (EW) theory and Quantum Chromodynamics (QCD), which explain the effects of the electromagnetic, strong and weak interactions. The gravitational interaction is negligible at the energy available in the current experiments $\left(<10^{3} \mathrm{GeV}\right)$.

The electroweak theory is an $\mathrm{SU}(2) \mathrm{xU}(1)$ local gauge theory with spontaneous symmetry breaking, which unifies the electromagnetic and the weak interactions. Its basic constituents are the three generations of quarks and leptons. It has one massless vector boson corresponding to the photon and three massive vector bosons, the $Z$ and 
the $W^{+}$and $W^{-}$bosons, that generate the neutral and charged current interactions. These massive bosons acquire mass through spontaneous symmetry breaking (the Higgs mechanism), which also generates mass for the fermions - quarks and leptons.

Quantum Chromodynamics is an $\mathrm{SU}(3)$ gauge theory describing the strong interactions amongst the quarks and gluons. Its predictive power depends on the energy scale. The short distance effects, corresponding to interactions at energy transfers significantly higher than the QCD scale $\left(\Lambda_{Q C D} \approx 0.2 \mathrm{GeV}\right)$, can be calculated perturbatively to any order of the perturbation theory. The long distance effects on the other hand cannot be calculated perturbatively and other methods such as QCD sum rules, lattice QCD, approximate QCD symmetries are employed to determine interaction properties. However, these calculations in most cases have limited precision and are quite challenging.

The Lagrangian of the QCD is invariant under the charge conjugation $(\mathrm{C})$, parity $(\mathrm{P})$ and time inversion $(\mathrm{T})$ operations. These discrete symmetries are an essential part of the Standard Model. Based on basic principles such as Lorentz invariance it can be shown that in any reasonable field theory the combined CPT symmetry is conserved [1].

In the weak interactions $\mathrm{C}$ and $\mathrm{P}$ symmetries are not conserved. For example in the limit of massless neutrino only left-handed neutrinos and right-handed antineutrinos take part in the weak interactions. The combined $C P$ symmetry, which transforms a particle into its antiparticle with opposite helicity, is also broken in the 
Standard Model, although to a much smaller degree.

In the Standard Model, the breaking of the $C P$ symmetry is allowed due to the presence of a single non-trivial phase in the three generation quark mixing matrix the Cabibbo-Kobayashi-Maskawa (CKM) matrix, which is a unitary matrix and can be presented in the following form using the Wolfenstein parameterization [2]:

$$
V_{C K M}=\left(\begin{array}{ccc}
V_{u d} & V_{u s} & V_{u b} \\
V_{c d} & V_{c s} & V_{c b} \\
V_{t d} & V_{t s} & V_{t b}
\end{array}\right)=\left(\begin{array}{ccc}
1-\lambda^{2} / 2 & \lambda & A \lambda^{3}(\rho-i \eta) \\
-\lambda & 1-\lambda^{2} / 2 & A \lambda^{2} \\
A \lambda^{3}(1-\rho-i \eta) & -A \lambda^{2} & 1
\end{array}\right)+O\left(\lambda^{4}\right)
$$

where $\lambda=\sin \theta \approx 0.22$ and $\theta$ is the Cabibbo angle. The parameters $A, \rho$ and $\eta$ are real numbers of the order of unity, which according to the latest CKM fit results [3] are $0.81 \pm 0.03,0.22 \pm 0.06$ and $0.35 \pm 0.03$ respectively. A non-zero value for $\eta$ is required for breaking of the CP symmetry in the Standard Model. This parameterization of the CKM matrix is assumed for all further discussions.

The unitarity of the CKM matrix can be presented in the relations such as equation 2.2, which in the complex plane corresponds to a triangle (figure 2.1):

$$
V_{u d} V_{u b}^{*}+V_{c d} V_{c b}^{*}+V_{t d} V_{t b}^{*}=0 .
$$


The three angles of the unitary triangle are denoted by $\alpha, \beta$ and $\gamma$ :

$$
\alpha \equiv \arg \left[-\frac{V_{t d} V_{t b}^{*}}{V_{u b} V_{u b}^{*}}\right], \quad \beta \equiv \arg \left[-\frac{V_{c d} V_{c b}^{*}}{V_{t d} V_{t b}^{*}}\right], \quad \gamma \equiv \arg \left[-\frac{V_{u d} V_{u b}^{*}}{V_{c d} V_{c b}^{*}}\right] .
$$

In this notation $V_{u b} \propto e^{-i \gamma}$ and $V_{t d} \propto e^{-i \beta}$. See [5] for details.

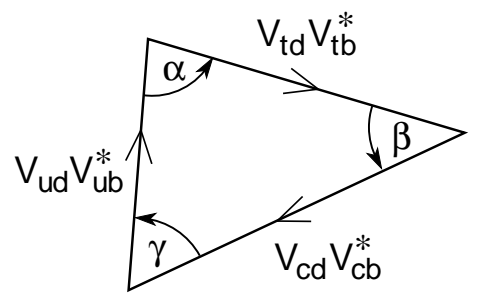

(a)

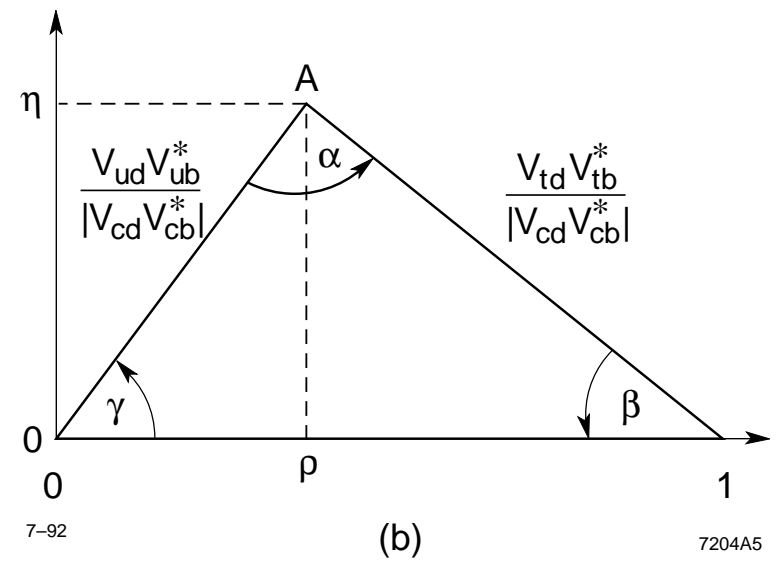

Figure 2.1: The unitarity triangle

\section{2 $C P$ violation}

There are three major types of $C P$ violating effects that can be observed in an experiment. Direct $C P$ asymmetry measures the difference in the decay rates for a particle and an antiparticle to the corresponding charge-conjugate final states, 


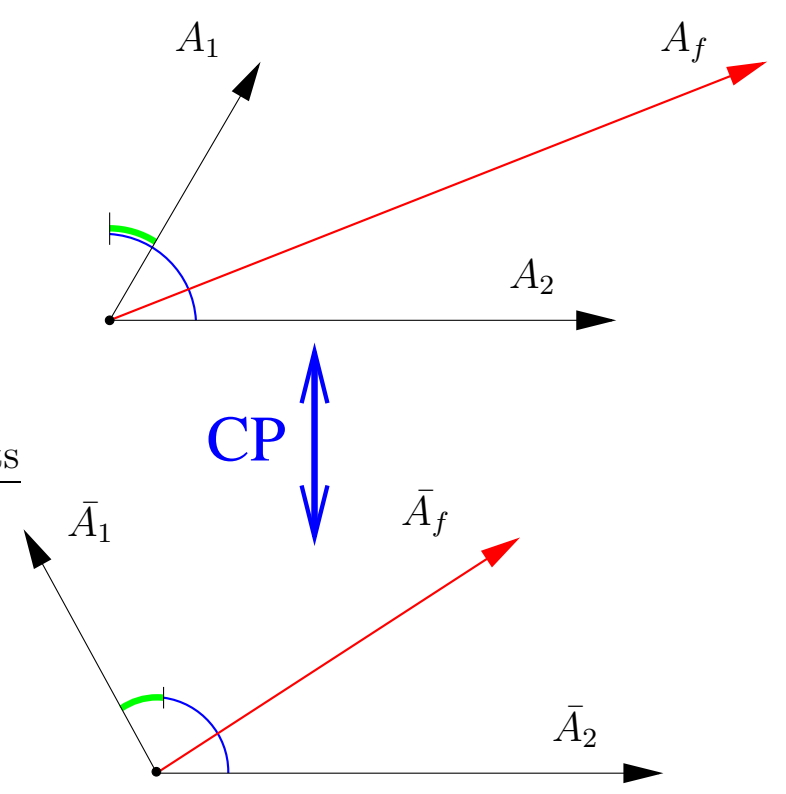

Figure 2.2: Direct $C P$ violation in the case of a decay with two dominant amplitudes $A_{1}$ and $A_{2}$ with non-zero strong (blue) and weak (green) relative phases. Since the sign of the weak phase is opposite for a particle and its anti-particle, the decay rates proportional to $\left|A_{f}\right|^{2}$ and $\left|\bar{A}_{f}\right|^{2}$ are different.

$A_{C P} \equiv \frac{\mathcal{B}(\bar{B} \rightarrow \bar{f})-\mathcal{B}(B \rightarrow f)}{\mathcal{B}(\bar{B} \rightarrow f)+\mathcal{B}(B \rightarrow f)}$. At first it was observed in the neutral kaon decays [6] at the level of a few parts per million and recently it has been observed in neutral B meson decays, in the decay mode $B^{0} \rightarrow K^{+} \pi^{-}[7]$, at a much higher level $\left(\left|A_{C P}\right|=0.13 \pm 0.03\right)$. This type of $C P$ violation is possible only if at least two different amplitudes contribute to the overall decay rate and they have non-zero relative weak and strong phases. Figure 2.2 illustrates the mechanism of direct $C P$ violation.

The other two types of $C P$ violation involve $B^{0}-\bar{B}^{0}$ mixing, which is a significant effect on the scale of the $B$ meson lifetime. The light $B_{L}$ and heavy $B_{H}$ mass 
eigenstates can be expressed in terms of the flavor eigenstates as:

$$
\begin{aligned}
& \left|B_{L}\right\rangle=p\left|B^{0}\right\rangle+q\left|\bar{B}^{0}\right\rangle \\
& \left|B_{H}\right\rangle=p\left|B^{0}\right\rangle-q\left|\bar{B}^{0}\right\rangle
\end{aligned}
$$

where the complex coefficients, $p$ and $q$, are bound by the normalization condition $|p|^{2}+|q|^{2}=1$ and the overall phase, $\arg \left(q / p^{*}\right)$, has no effect on physical observables. If $|p / q|=1$, the mass eigenstates are also $C P$ eigenstates. The mass difference of the two mass eigenstates $\left(\Delta m_{B}=M_{H}-M_{L}\right)$ in the case of $B_{d}$ mesons is significantly larger than the decay rate difference $\left(\Delta \Gamma_{B}=\Gamma_{H}-\Gamma_{L}\right)$ for these two states. Neglecting the difference in the decay rates, the time evolution of a neutral $B$ meson, starting in $B^{0}$ or $\bar{B}^{0}$ state, can be expressed in the following form:

$$
\begin{aligned}
\left|B_{\text {phys }}^{0}(t)\right\rangle & =g_{+}(t)\left|B^{0}\right\rangle+(q / p) g_{-}(t)\left|\bar{B}^{0}\right\rangle \\
\left|\bar{B}_{\text {phys }}^{0}(t)\right\rangle & =(p / q) g_{-}(t)\left|B^{0}\right\rangle+g_{+}(t)\left|\bar{B}^{0}\right\rangle
\end{aligned}
$$

where

$$
\begin{gathered}
g_{+}(t)=e^{-i M t} e^{-\Gamma t / 2} \cos \left(\Delta m_{B} t / 2\right) \\
g_{-}(t)=i e^{-i M t} e^{-\Gamma t / 2} \sin \left(\Delta m_{B} t / 2\right)
\end{gathered}
$$

and $M=\frac{1}{2}\left(M_{H}+M_{L}\right)$. 
$C P$ violation in mixing arises if the mass eigenstates of a neutral meson are not the $C P$ eigenstates. It reveals itself in the asymmetry of the decay rates to flavor specific final states that would only occur via mixing. For example, in the case of the semileptonic decays $B^{0} \rightarrow l^{+}+X\left(\bar{B}^{0} \rightarrow l^{-}+X\right)$, decays to the charge-conjugate states $B^{0} \rightarrow l^{-}+X\left(\bar{B}^{0} \rightarrow l^{+}+X\right)$ are only possible through mixing. The asymmetry in the decay rate with mixing as defined below, indicates that $|p / q| \neq 1$ and therefore $C P$ is violated.

$$
\begin{aligned}
a_{\mathrm{sl}} & \equiv \frac{P\left(\bar{B}^{0} \rightarrow B^{0}\right)-P\left(B^{0} \rightarrow \bar{B}^{0}\right)}{P\left(\bar{B}^{0} \rightarrow B^{0}\right)+P\left(B^{0} \rightarrow \bar{B}^{0}\right)} \\
& =\frac{\Gamma\left(\bar{B}_{\mathrm{phys}}^{0}(t) \rightarrow l^{+} \nu X\right)-\Gamma\left(B_{\mathrm{phys}}^{0}(t) \rightarrow l^{-} \bar{\nu} X\right)}{\Gamma\left(\bar{B}_{\mathrm{phys}}^{0}(t) \rightarrow l^{+} \nu X\right)+\Gamma\left(B_{\mathrm{phys}}^{0}(t) \rightarrow l^{-} \bar{\nu} X\right)} \\
& =\frac{\left|(p / q) g_{-}(t) A^{*}\right|^{2}-\left|(q / p) g_{-}(t) A\right|^{2}}{\left|(p / q) g_{-}(t) A^{*}\right|^{2}+\left|(q / p) g_{-}(t) A\right|^{2}}=\frac{1-|q / p|^{4}}{1+|q / p|^{4}} .
\end{aligned}
$$

It is also possible to measure $C P$ violation in mixing in inclusive $B$ meson decays without using the final state flavor tagging:

$$
\frac{N\left(B^{0}(t) \rightarrow \text { all }\right)-N\left(\bar{B}^{0}(t) \rightarrow \text { all }\right)}{N\left(B^{0}(t) \rightarrow \text { all }\right)+N\left(\bar{B}^{0}(t) \rightarrow \text { all }\right)} \simeq a_{\mathrm{sl}}\left[\frac{\Delta m_{d}}{2 \Gamma_{d}} \sin \left(\Delta m_{d} t\right)-\sin ^{2}\left(\frac{\Delta m_{d} t}{2}\right)\right]
$$

The most accurate measurements of $C P$ violation in mixing is achieved with the inclusive dilepton events [4], which represent about $4 \%$ of all $B \bar{B}$ decays. The flavor of each $B$ meson is tagged by the charge of the lepton. The asymmetry between same-sign lepton pairs, $l^{+} l^{+}$and $l^{-} l^{-}$, allows to measure the asymmetry in the decay 
rate:

$$
a_{\mathrm{sl}}^{\text {meas }}(\Delta t)=\frac{N\left(l^{+} l^{+}, \Delta t\right)-N\left(l^{-} l^{-}, \Delta t\right)}{N\left(l^{+} l^{+}, \Delta t\right)+N\left(l^{-} l^{-}, \Delta t\right)}=a_{\mathrm{sl}} \frac{S(\Delta t)}{S(\Delta t)+B(\Delta t)}
$$

where $S(\Delta t)$ and $B(\Delta t)$ are the number of signal and background events respectively.

The world average of the asymmetry measurements gives [39]

$$
\left|\frac{q}{p}\right|=1.0013 \pm 0.0034 .
$$

$C P$ violation also arises in interference between decay amplitudes with and without mixing into final states that can be reached by both $B^{0}$ and $\bar{B}^{0}$ (See figure 2.3). The $B^{0} \bar{B}^{0}$ system from the $\Upsilon(4 S)$ decay, propagates in a coherent state with exactly one $B^{0}$ and one $\bar{B}^{0}$ mesons until one of the mesons decays. If we know the flavor of one $B$ meson at the decay time, then we know the flavor of the other $B$ at this time as well. The time evolution of the other $B$ meson follows

$$
f=\frac{\Gamma}{4} e^{-\Gamma|\Delta t|}[1 \pm(-C \cos (\Delta m \Delta t)+S \sin (\Delta m \Delta t))]
$$

where the sign is positive for a $B^{0} \operatorname{tag}$ and negative for a $\bar{B}^{0}$ tag. The cosine term, $C$, is related to the direct $C P$ violation and the sine term, $S$, is a measure of the $C P$ violation due to the interference of the decay amplitudes with and without mixing. The typical asymmetries observed in experimental measurements can be seen in figure 2.4, which shows the $C P$ violation in the case of charmonium $(b \rightarrow c \bar{c} s)$ decays. In the Standard Model the $C P$ violating asymmetry for the decay $B^{0} \rightarrow J / \psi K^{0}$ (the so called "gold- 


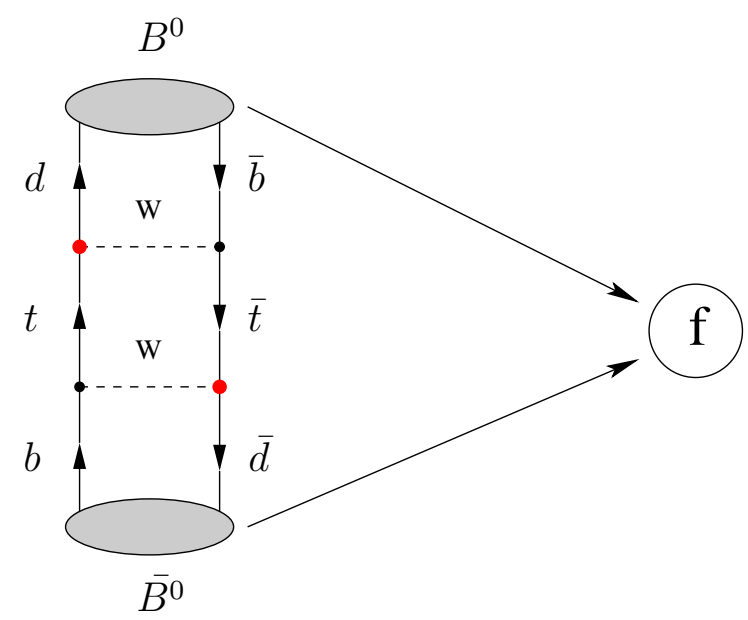

Figure 2.3: Time-dependent $C P$ violation is caused by interference of two amplitudes, which correspond to decay with and without mixing. The phase difference due to mixing is $e^{-i 2 \beta}$ and in some cases the decay amplitudes might also contribute to it. The relative contribution of each amplitude varies with time due to $B^{0}-\bar{B}^{0}$ oscillation.

plated" mode) is dominated by a single weak phase $(-2 \beta)$ and corrections to this phase either in mixing or the decay amplitude itself are small. In the Wolfenstein parameterization the leading order amplitudes carry zero relative weak phases, and only a suppressed penguin amplitude, with a suppression factor of the order $\lambda^{2} \sim$ $O\left(10^{-2}\right)$ has non-zero weak phase, which may have a small impact on the measured asymmetry in $B^{0} \rightarrow J / \psi K^{0}$. The direct $C P$ asymmetry is expected to be zero since the dominant amplitudes have zero relative weak phases. [8]

\subsection{Physics beyond the Standard Model}

The Standard Model can predict or at least does not contradict all experimental data currently available, but there are reasons to believe that the Standard Model is an 


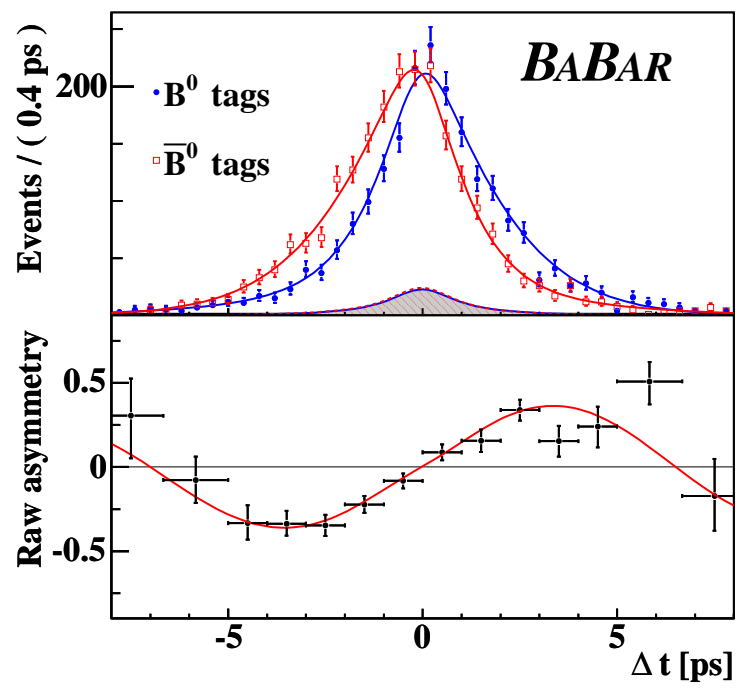

Figure 2.4: The time-dependent $C P$ violation for charmonium decays based on Run 1-4 data (232 million $B \bar{B}$ pairs) collected by the BaBar detector at SLAC.

effective theory for some more fundamental theory, which can ultimately unite all types of interactions known so far.

The most compelling evidence for the existence of physics beyond the Standard Model comes from astrophysics. For many decades it was known that the visible mass of stars and galaxies is not sufficient to explain astronomical observations such as the galaxy velocity distribution in galaxy clusters, which indicate an existence of some unknown type of matter in the Universe. Recent experimental results support this point of view even stronger. The gravitational lensing effect of the light deflection of foreground astronomical objects in the gravitational field of massive dark matter formations, made it possible to measure the mass distribution in the clusters.

The cosmic microwave background(CMB) anisotropy measurements helped to conclude that our universe has a flat geometry and led to a confirmation of the Standard 
Model of Cosmology and provided an evidence for inflation in the evolution of Universe. According to these observations the dark matter represents about $21 \%$ of the total mass of the universe and the dark energy is about $75 \%$, which leaves only about $4 \%$ to the visible mass of the universe.

The most striking discovery was the fact that the universe expands with increasing rate. Accelerating expansion of the universe implies existence of some unknown energy (dark energy) that overcomes gravitational attraction. More observations of supernovae with wide range of redshifts are necessary to solidify theses conclusions.

The Standard Model on its own has some theoretical difficulties of self-consistency such as the Higgs mass divergence due to loop corrections - the hierarchy problem. The problem arises from the fact that the Higgs mass receives quadratically divergent contributions from the top, the vector bosons and the Higgs loop diagrams. This makes the Higgs mass too large, unless the tree and loop contributions to the Higgs mass are fine-tuned to cancel the effect. ${ }^{1}$ If one assumes that the Higgs mass is of the order of $200 \mathrm{GeV}$ and the Standard Model holds at least to the level of $10 \mathrm{TeV}$, the required degree of fine-tuning is one part in 100 [9]. Even though the fine-tuning itself does not invalidate any model, a high degree of fine-tuning makes a model unlike candidate for a fundamental description of the nature.

\footnotetext{
${ }^{1}$ The term fine-tuning refers to the unnatural model parameters selection required to keep a theoretical model consistent with some fundamental principles or experimental observations. The naturalness is defined by Gerard 't Hooft(1979) as: A theory obeys naturalness only if all of its small parameters would lead to an enhancement of its exact symmetry group when replaced by zero. In other words, at any energy scale $\mu$, a physical parameter or set of parameters $\alpha_{i}(\mu)$ is allowed to be very small only if the replacement $\alpha_{i}(\mu)=0$ would increase the symmetry of the system.
} 
Many new ideas and models have been proposed to extend the Standard Model, including grand unified theories and super-symmetric theories. The super-symmetry predicts existence of many new particles - super partners of know particles (each boson gets a new fermion and each fermion gets a new boson counterpart), but even if the super-symmetry is not realized in nature many other extensions of the Standard Model predict new particles yet to be observed.

Even if the masses of the new particles are significantly higher than the currently available energies of the accelerators, we can still probe the effect of new physics through precise measurements of rare processes in which the effect of new physics can be present through loop diagrams involving virtual particles.

\subsection{Phenomenology of $B$ meson decays}

A successful search for new physics effects requires exceptional experimental sensitivity and the ability to calculate theoretical predictions with matching precision. In the case of $\mathrm{B}$ meson decays the energy scale of different processes is close to $\Lambda_{Q C D} \approx 0.2 \mathrm{GeV}$ - the scale below which the QCD enters non-perturbative regime and the theory predictions might lack required precision to make use of available high precision data. For example the hadronization of the final state particles in most cases cannot be calculated in the framework of perturbative QCD. In fact the $b$ quark itself is found in a bound state with other light quarks, which essentially makes low energy effects unavoidable. Different methods are used to achieve reliable theory predictions. 
Lattice QCD [10] in principle can be used to calculate any process in QCD with high precision, but in practice, at this point, this method has limited predictive power. It is expected that with more computing resources available, the predictive power of Lattice QCD will grow. Currently a state-of-the-art calculation uses on the order of $10^{18}-10^{19}$ floating point operations, whereas the overall computing resources allocated for the US Lattice QCD community are capable of $\approx 10^{19}$ floating point operations per year. Another essential issue is reliability of the error estimation for Lattice QCD, which is once again connected to the available computing power.

Perturbative calculations in QCD can be done in the framework of the Operator Product Expansion (OPE) [11]. The OPE simplifies the theoretical calculations by introducing a framework, where all heavy particles with mass more than the mass of the $b$ quark are integrated out (heavy vector bosons and the top quark), which gives an effective model with only 5 quarks and an effective Hamiltonian of the following form:

$$
H_{e f f}=-\frac{4 G_{F}}{\sqrt{2}} \sum \lambda_{i}^{C K M} C_{i}(\mu) O_{i}(\mu)=-\frac{4 G_{F}}{\sqrt{2}} V_{t b} V_{t s}^{*} \sum C_{i}(\mu) O_{i}(\mu) .
$$

This allows for a scale separation, where the long-distance effects are contained in the operator $O_{i}(\mu)$ matrix elements and the short-distance effects in the so-called Wilson coefficients $C_{i}(\mu)$ calculated perturbatively. Since the $W$ mass is much bigger than the $b$-quark mass, the effective interaction can be seen as a four fermion local interaction with the expansion factor $p^{2} / M_{W}^{2}$, where $p$ is a typical momentum transfer and $M_{W}$ 
- mass of the $W$ boson. This method makes it possible to obtain predictions in the case of the inclusive $B$ meson decays, where the non-perturbative QCD contributions play only a subdominant role and can be calculated using other approaches.

Many different theoretical techniques have been developed to calculate QCD contribution with virtualities at the level of the $b$ quark mass. The most successful approach so far is to use different limits and symmetry arguments of QCD and when possible to calculate perturbatively deviations from these limits or symmetries. In the case of $B$ meson decays the Heavy-Quark Effective Theory (HQET) [12] and the Chiral Perturbation Theory (ChPT) [13] play important roles. The HQET represents the limit of infinite mass of $c$ and $b$ quarks, which essentially makes the heavy quark look like a static source of electric and color fields. The chiral symmetry of QCD assumes that all light quarks are massless and allows to achieve high precision predictions for the case of small external momentum of final state particles.

One widely used method is based on the factorization principle, which assumes that it is possible to factorize contributions from short-distance and long-distance effects. For example the matrix element for the decay $B \rightarrow K^{0} \pi^{0}$ would factorize as:

$$
\left\langle B^{0}|Q| K^{0} \pi^{0}\right\rangle \approx\left\langle B^{0}\left|j_{1}\right| K^{0}\right\rangle\left\langle 0\left|j_{2}\right| \pi^{0}\right\rangle \sim F^{B^{0} \rightarrow K^{0}}\left(m_{K^{0}}^{2}\right) f_{\pi^{0}}
$$

where $F^{B^{0} \rightarrow K^{0}}\left(m_{K^{0}}^{2}\right)$ is the form factor corresponding to $B^{0} \rightarrow K^{0}$ transition and $f_{\pi}$ is the pion decay constant.

This approach is based on the idea that the light meson, which does not have 
the spectator quark from the original heavy $B$ meson can be seen as a color dipole with a small dipole moment that makes it essentially color "transparent" and its interaction with the medium can be neglected. This idea was formulated by Bjorken in the 80's [14]. Unfortunately this approach allows only for very rough estimations of the decay rates, because it essentially neglects the exchange of "non-factorizable" gluons between the light meson formation process and the $B$ meson decay. It does not provide any physical mechanism that could account for rescattering in the final state and for the generation of a strong phase shift between different amplitudes.

A more rigorous approach to the factorization idea is provided in the framework of the QCD factorization [15], which concludes that "non-factorizable" corrections are dominated by hard gluon exchange, while the soft effects that survive in the heavyquark limit are confined to the meson that picks up the spectator quark in the $B$ meson. In this case the factorization formula becomes

$$
\left\langle B^{0}|Q| K^{0} \pi^{0}\right\rangle=\left\langle B^{0}\left|j_{1}\right| K^{0}\right\rangle\left\langle 0\left|j_{2}\right| \pi^{0}\right\rangle\left[1+\sum r_{n} \alpha_{s}^{n}+\mathcal{O}\left(\Lambda_{Q C D} / m_{b}\right)\right]
$$

where $\alpha_{s}$ is the strong coupling constant. The result is valid up to corrections suppressed by $\Lambda_{Q C D} / m_{b}$. This approach allows to calculate not only the decay amplitudes, but the strong phases as well. At this time the predictive power of the model in calculating the strong phases is not very well tested in experiments, so for now we should take such predictions with some caution. 


\subsection{Experimental observables}

The choice of optimal experimental observables is driven by the accuracy of the theoretical predictions and the experimental feasibility to extract this information from available data. Quite often the quantities that can be precisely calculated are very hard to measure and vise versa.

First of all new physics can reveal itself in the measurements of the decay rates. The main drawback of this approach is the limited precision of the theoretical predictions of the decay amplitudes that contribute to the overall decay rate in the Standard Model. For example the branching ratio of $B \rightarrow K^{*} \gamma$ is measured experimentally with much better precision than it can be calculated in the Standard Model.

One of the ways to improve the situation is to measure inclusive decay rates. This way the low energy effects can be factorized out and assuming that hadronization of the final state has unit probability one can conclude that the decay amplitude is dominated by short-distance effects, which can be calculated perturbatively. This argument only holds for energy transfer to the final state particles, which is significantly higher than $\Lambda_{Q C D}$ scale. The most interesting inclusive $B$ decays $B \rightarrow X_{s, d} \gamma$, $B \rightarrow X_{s} l^{+} l^{-}$and $B \rightarrow X_{s} \nu \bar{\nu}$, where $X$ is an inclusive hadronic state containing no charmed particles, are well studied and have relatively clean theoretical predictions [16].

A similar problem is encountered in predictions of direct $\mathrm{CP}$ violating asymmetries. As it was mentioned earlier direct $\mathrm{CP}$ violation occurs only if at least two 
amplitudes with different weak and strong phases contribute to the decay rate. The strong phase emerges as a result of long distance strong interaction and therefore the theoretical calculations of direct $\mathrm{CP}$ violation are quite limited in precision. Nevertheless direct $\mathrm{CP}$ violation searches can be sensitive to new physics, especially when the expected CP violation in the Standard Model is close to zero due to the presence of only one dominant decay amplitude. The new physics might enhance the relative size of the suppressed amplitude and result in a sizable direct $\mathrm{CP}$ violation.

The $S$ coefficient of the time-dependent $C P$ violating asymmetry (equation 2.14), represents a unique observable, which can be measured with high precision and is theoretically clean in a number of exclusive $B$ meson decays. By definition

$$
\begin{aligned}
\lambda & =\left(\frac{q}{p} \frac{\bar{A}_{\bar{f}_{C P}}}{A_{f_{C P}}}\right) \\
S & =-\frac{2 \operatorname{Im} \lambda}{1+|\lambda|^{2}} .
\end{aligned}
$$

In the Wolfenstein parameterization of the CKM matrix only two elements, $V_{t d}$ and $V_{u b}$, have non-zero weak phases. If two different amplitudes contribute to the decay amplitude and they carry the same weak phase the absolute value of the decay amplitude cancels out and the $S$ term is free from the QCD uncertainties on the relative size of the amplitudes. This allows to detect unambiguously a small deviation from the expected asymmetry, which can be attributed to a new physics contribution to the decay amplitude. For example in the case of $B^{0} \rightarrow J / \psi K_{S}$ there are two leading 
order amplitudes proportional to $\lambda^{2}$ with zero weak phases and one sub-leading order amplitude $\left(\lambda^{4}\right)$ that carries a weak phase. The effect of this weak phase is suppressed at the level of $\lambda^{4} / \lambda^{2} \approx 5 \%$. New physics contribution might change the asymmetry if the new physics amplitude has a different weak phase with respect to the dominant amplitudes.

Another observable that can be used for new physics searches is the polarization of the final state particles. Since the weak current couples only to left chirality fields in the limit of $m / E \rightarrow 0$ one can observe that decay products are polarized $(B \rightarrow \rho \rho$, $\left.B \rightarrow \phi K^{*}, B \rightarrow K^{*} \gamma\right)$. Unfortunately hadronization effects might play a significant role, hence making it difficult to get a reliable theoretical prediction.

\subsection{CP violation in $B^{0} \rightarrow K^{0} \pi^{0}$}

The $B^{0} \rightarrow K^{0} \pi^{0}$ decay is dominated by a single penguin amplitude (Figure 2.5) with a top quark in the loop, which in the Wolfenstein parameterization, neglecting terms of order $O\left(\lambda^{4}\right)$, has zero weak phase. The tree amplitude is doubly Cabibbo and color suppressed. Correction due to other lighter quarks in the loop can be neglected, since their contributions are roughly proportional to $m_{q}^{2} / m_{W}^{2}\left|V_{q s}^{*} V_{q b}\right|$, where $m_{q}$ and $m_{W}$ are masses of the quark and the $W$ boson respectively.

Since the dominant decay amplitude carries zero weak phase, the $S$ term of the time-dependent $C P$ asymmetry in this decay channel is expected to be the weak phase of the $B^{0}-\bar{B}^{0}$ mixing, which is measured with a good precision in the $B$ meson decays 
to charmonium states. The $C P$ eigenvalue of the final state is simply a product of the $C P$ eigenvalues of the $K_{S}$ and $\pi^{0}$, which are +1 and -1 , respectively. In the case of $B^{0} \rightarrow K_{L} \pi^{0}$ the final state has the opposite $C P$ eigenvalue, but this final state is experimentally inaccessible for time-dependent asymmetry measurements, hence only the $K_{S} \pi^{0}$ final state is considered. Using the Heavy Flavor Averaging Group (HFAG) world average of the $\sin 2 \beta$ measurements [39], in the leading order the expected $C P$ asymmetry in this mode is:

$$
S=-\eta_{C P} \sin 2 \beta=0.69 \pm 0.03
$$

Next order approximation of the CKM matrix elements allows a non-zero weak phase in the penguin process, which is suppressed to few percent level:

$$
V_{t s}=-A \lambda^{2}+\frac{1}{2} A \lambda^{4}[1-2(\rho+i \eta)] \approx-A \lambda^{2} e^{i \beta_{S}}
$$

where $\beta_{S} \approx 0.018 \mathrm{rad} \approx 1.05^{\circ}$. This leads to a small shift in the expected $S_{K_{S} \pi^{0}}$ :

$$
S_{K_{S} \pi^{0}}=-\eta_{C P} \sin \left(2 \beta+2 \beta_{S}-2 \delta_{\text {tree }}\right) \approx \sin 2 \beta+0.025+\Delta S_{\text {tree }}
$$

where $\delta_{\text {tree }}$ is the suppressed tree amplitude contribution to the overall weak phase of the decay amplitude and $\Delta S_{\text {tree }}$ is its effect on expected value of the $S$ term. Since current experimental and theoretical errors are significantly larger than the 

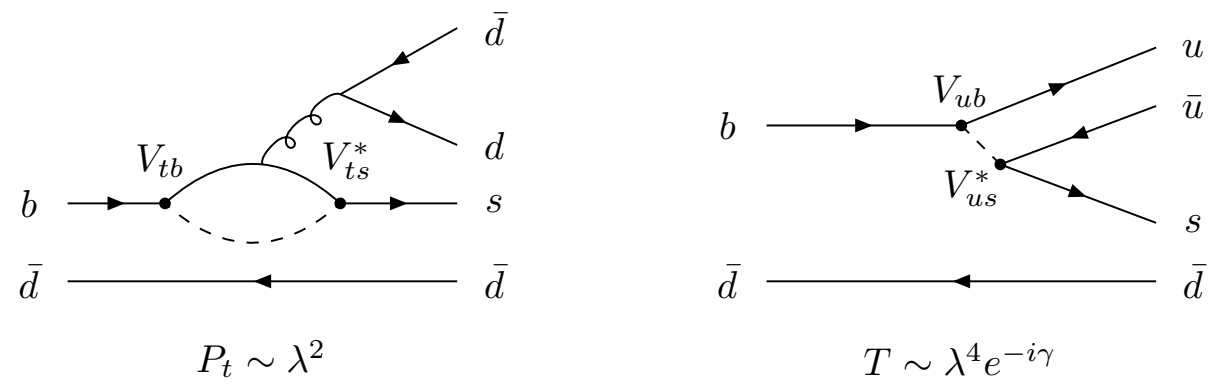

Figure 2.5: The dominant penguin and suppressed tree diagrams of $B^{0} \rightarrow K^{0} \pi^{0}$

contribution of the $\beta_{S}$, in most calculations it is ignored.

The sub-leading order tree amplitude is both Cabibbo and color suppressed. Since the tree diagram carries a different weak phase from the penguin process, it can result in deviation of $S$ from $-\eta_{f} \sin 2 \beta$. In order to estimate the relative size of its amplitude and the overall weak phase, one can use different theoretical models or symmetries of QCD. These are discussed in the following sections and estimates are presented for the deviation $\Delta S \equiv S_{K_{S} \pi^{0}}-\sin 2 \beta$

\subsubsection{Estimation of the sub-leading amplitude effect on the expected $C P$ asymmetry using $\mathrm{SU}(3)$ approximation}

Using SU(3) flavor symmetry it is shown [17] that in the Standard Model the maximal deviation of the expected asymmetry from $\sin (2 \beta)$ is less than 0.2 . The basic idea of this approach is to relate the $B^{0} \rightarrow K^{0} \pi^{0}$ decay to $B^{0} \rightarrow \pi^{0} \pi^{0}$, which has the same two leading order amplitudes, but the tree amplitude is dominant, whereas the penguin amplitude is suppressed.

The decay amplitudes can be decomposed in terms of the basic diagrams in the 
following way [18]:

$$
\begin{gathered}
A_{\pi^{0} K^{0}}=-\frac{C^{\prime}}{\sqrt{2}}+\frac{P^{\prime}}{\sqrt{2}} \\
A_{\pi^{0} \pi^{0}}=-\frac{C}{\sqrt{2}}+\frac{P}{\sqrt{2}}+\frac{E}{\sqrt{2}}+\frac{P A}{\sqrt{2}}
\end{gathered}
$$

where $C$ is a color-suppressed tree amplitude, $P$ is a penguin amplitude with virtual quarks of charge $2 / 3$ in the loop, $E$ is an exchange amplitude, $A$ is an annihilation amplitude contributing only to charged $B$ decays and $P A$ is a penguin annihilation amplitude. (See corresponding diagrams in figure 2.6). The unprimed diagrams correspond to strangeness conserving decays and the primed ones correspond to strangeness violating decays. Assuming SU(3) symmetry, the primed and unprimed amplitudes are related in the following way:

$$
\frac{C^{\prime}}{C}=\frac{E^{\prime}}{E}=\frac{A^{\prime}}{A}=\frac{V_{u s}}{V_{u d}} \approx \lambda \approx 0.23
$$

and

$$
\frac{P^{\prime}}{P}=\frac{P A^{\prime}}{P A}=\frac{V_{t s}}{V_{t d}} \approx 5
$$

Taking into account these relations and using the following branching ratios as input[39]:

$$
\begin{aligned}
B\left(B^{0} \rightarrow \pi^{0} \pi^{0}\right) & =(1.45 \pm 0.29) \times 10^{-6} \\
B\left(B^{0} \rightarrow K^{0} \pi^{0}\right) & =(11.5 \pm 1.0) \times 10^{-6}
\end{aligned}
$$

one can get a bound on the relative size of the two amplitudes contributing to $B^{0} \rightarrow$ 


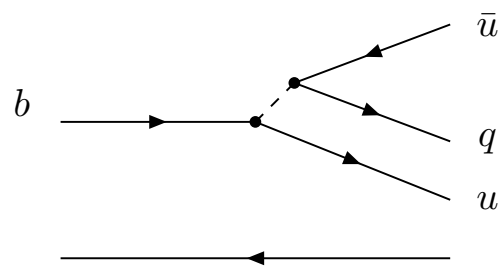

$T, T^{\prime}$

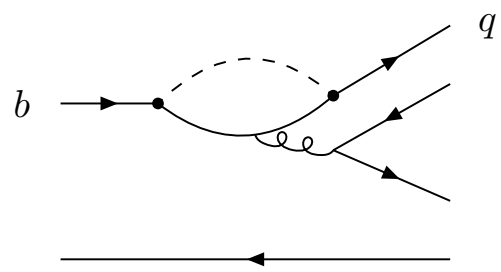

$$
P, P^{\prime}
$$

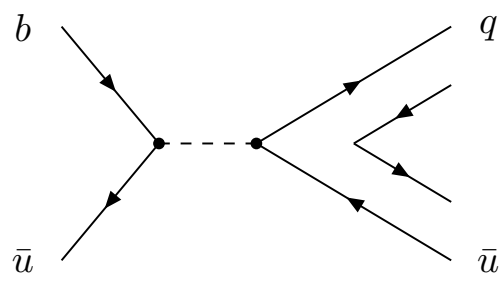

$A, A^{\prime}$

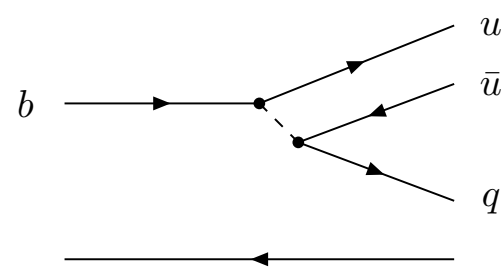

$C, C^{\prime}$

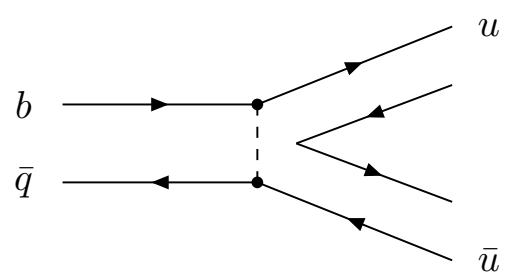

$E, E^{\prime}$

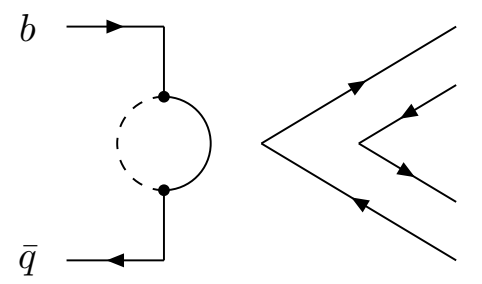

$P A, P A^{\prime}$

Figure 2.6: Diagrams contributing to charmless $\bar{B}$ meson decays 
$K^{0} \pi^{0}$ decay. Combing this result with known limits on the $\gamma$ angle and taking into account possible corrections due to $\mathrm{SU}(3)$ breaking, which normally are at the level of $20-30 \%$, it is found that:

$$
\left|\Delta S_{\pi K}\right|=\left|S_{B^{0} \rightarrow K^{0} \pi^{0}}-\sin 2 \beta\right|<0.2
$$

where all experimental values are taken at the $90 \%$ confidence limits.

Contributions of $E, A$ and $P A$ diagrams can normally be neglected in the case of charmless $B$ decays, since the corresponding amplitudes are suppressed by a factor of $f_{B} / m_{B} \sim 1 / 20$, where $f_{B}$ is the $B$ meson decay constant, and in addition diagrams $E$ and $A$ are also helicity suppressed. Since the $B^{0} \rightarrow \pi^{0} \pi^{0}$ decay amplitude contains the exchange and the penguin annihilation diagrams, one can verify that their contribution is negligible by looking for the $B^{0} \rightarrow K^{+} K^{-}$decays, which can only proceed through the exchange or the penguin annihilation amplitudes. Currently the upper limit (at $90 \%$ C.L.) on the branching fraction of the $B^{0} \rightarrow K^{+} K^{-}$is at $0.6 \times 10^{-6}[19]$. This measurement is based on a sample of 88 million $B \bar{B}$ pairs collected by the BaBar detector. A recent measurement by BABAR collaboration [20], presented at EPS International Europhysics Conference on High Energy Physics, based a sample of 227 million $B \bar{B}$ pairs, sets an upper limit to $0.4 \times 10^{-6}$ at a $90 \%$ confidence limit. Event though the bound is not very strong, a more detailed calculation, carried out in [17], shows that the possible contribution of the neglected $E$ and $P A$ amplitudes is small.

In general, neglecting the $\mathrm{SU}(3)$ breaking effects, the sensitivity of $\mathrm{SU}(3)$ approach 
is limited to $\left|P^{\prime} / C^{\prime}\right| \gtrsim \lambda^{2}$, which comes from the ratios of primed and not primed tree and penguin amplitudes and leads to a limit on the sensitivity to $\Delta S$ :

$$
\left|\Delta S_{S U(3)}\right| \simeq 2\left|C^{\prime} / P^{\prime}\right| \cos 2 \beta \cos \delta \sin \gamma \gtrsim 0.06 .
$$

This method can hardly give a significantly better estimate on $\Delta S_{\pi K}$ than the current prediction, $\left|\Delta S_{\pi K}\right|<0.2$, unless the direct $\mathrm{CP}$ violation is observed in $B^{0} \rightarrow K^{0} \pi^{0}$ or some other way a better bound on the relative strong phase can be found. This issue can be resolved by relating to other decay modes when more precise measurements of the branching ratios of $B \rightarrow P P$ charmless decays are available. Unfortunately even in this case the $\mathrm{SU}(3)$ symmetry breaking will limit the sensitivity of this method.

One of the possible ways to improve the prediction is to use isospin symmetry instead of SU(3) symmetry and bound the size of the suppressed tree amplitude by comparing the decay rates of $B^{0} \rightarrow K^{0} \pi^{0}$ and $B^{+} \rightarrow K^{0} \pi^{+}$. The decay amplitude of $B^{+} \rightarrow K^{0} \pi^{+}$can be expressed in the following form:

$$
A_{\pi K^{0}}=P^{\prime}+A^{\prime}
$$

As it was mentioned earlier the $A^{\prime}$ amplitude is doubly suppressed and can be ignored or bounded using $\mathrm{SU}(3)$ and the branching ratio of $B^{+} \rightarrow K^{+} \bar{K}^{0}$ decay. In this case $B^{+} \rightarrow K^{0} \pi^{+}$decay amplitude is simply $P^{\prime}$ and it is possible to estimate the size of 
the suppressed tree amplitude $\left(C^{\prime}\right)$. This approach requires precise measurements of the branching ratios of $B^{0} \rightarrow K^{0} \pi^{0}$ and $B^{+} \rightarrow K^{0} \pi^{+}$as well as the $\gamma$ angle of the unitarity triangle and direct $\mathrm{CP}$ violation in $B^{0} \rightarrow K_{S}^{0} \pi^{0}$.

\subsubsection{Estimation of the sub-leading amplitude effect on the expected $C P$ asymmetry using the QCD factorization approach}

Another way to estimate $\Delta S_{\pi K}$ is to use the QCD factorization approach [15]. Recent calculation of the expected $\Delta S_{\pi K}$ [21] using the QCD factorization approach, predicts that it should be positive and quite small:

$$
\Delta S_{\pi K} \in[+0.02,0.15]
$$

This calculation takes into account the experimental uncertainties on the input parameters of the model as well as theoretical errors, which were estimated by scanning the parameter's space of the model constraining the model predictions of the branching ratios to be within 3 standard deviations from experimental measurements of $B^{0} \rightarrow \pi^{0} K^{0}, B^{0} \rightarrow \rho^{0} K^{0}, B^{0} \rightarrow \eta K^{0}, B^{0} \rightarrow \omega K^{0}$.

The $\mathrm{CP}$ violating asymmetries in other $b \rightarrow s$ decays are approached in a similar manner, but each mode can have its own complications. Current experimental data shows some evidence of non-zero $\Delta S$ averaged over a few different $b \rightarrow s$ decay modes, 
but it requires a further theoretical analysis and more data for each decay mode for a conclusive evidence, or significant constraint, to emerge.

In summary it is expected that in the Standard Model $\left|\Delta S_{K_{S}^{0} \pi^{0}}\right|=\left|S_{K_{S}^{0} \pi^{0}}-\sin 2 \beta\right|$ is less than 0.1-0.2 and QCD factorization based calculations predict that $\Delta S_{K_{S}^{0} \pi^{0}}$ should be positive, i.e. $S_{K_{S}^{0} \pi^{0}}>\sin 2 \beta$. The branching ratio measurement of $B^{0} \rightarrow$ $K^{0} \pi^{0}$ enters most of these calculations, hence with improved uncertainties it can help in reducing the range of allowed $\Delta S$. The direct $\mathrm{CP}$ violation, on the other hand, typically provides a poor bound on parameters of different models, but if a sizable asymmetry is measured it can give some insight on the underlying physics.

\subsection{CP violation in $B^{0} \rightarrow K_{S}^{0} \pi^{0} \gamma$}

The decay amplitude of the rare decay $B^{0} \rightarrow K_{S}^{0} \pi^{0} \gamma$ is dominated by a top-quark mediated $\bar{b} \rightarrow \bar{s} \gamma$ radiative penguin, which carries zero weak phase in the Wolfenstein parameterization of the CKM matrix (figure 2.7). Since the final state is a $C P$ eigenstate, one expects that the time-dependent $C P$ symmetry violation in this decay is governed by $\sin 2 \beta$. However, since the $W$-boson couples only to left handed chirality fields, the photon is predominantly circularly polarized ( left-handed for $b \rightarrow s \gamma$ and right-handed for $\bar{b} \rightarrow \bar{s} \gamma$ ), hence the two final states are largely orthogonal and not reachable by both $B^{0}$ and $\bar{B}^{0}$ mesons. Therefore the $C P$ violation in the interference of mixing and decay nearly vanishes in the Standard Model [22].

Because the $B^{0} \rightarrow K_{S}^{0} \pi^{0} \gamma$ decay is dominated by a loop diagram, it may receive 


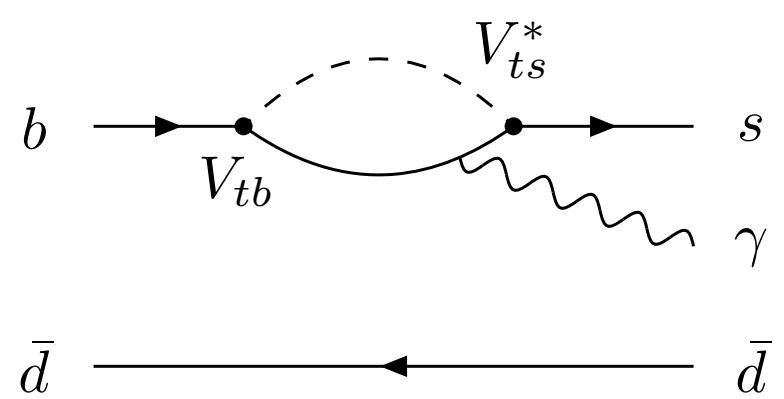

Figure 2.7: A leading order penguin diagram for $B^{0} \rightarrow K^{* 0} \gamma$

substantial contributions from physics beyond the standard model. Since the expected $C P$ violating asymmetry in the Standard Model is almost zero, this decay can be sensitive to new physics effects, which allow different photon polarizations and alter the $C P$ asymmetry from the Standard Model value.

To a good approximation a $\bar{B} \rightarrow \bar{X}_{s} \gamma$ decay can be viewed as a 2-body $b \rightarrow$ $s \gamma$ transition followed by the $s$-quark fragmentation. Taking into account angular momentum conservation and the fact that the mass of the $s$ quark is small compared to its energy $\left(\approx \frac{m_{b}}{2}\right)$, the ratio of the amplitudes for $b \rightarrow s \gamma_{R}$ and $b \rightarrow s \gamma_{L}$ in the Standard Model is:

$$
\mathcal{R}=\frac{A\left(b \rightarrow s \gamma_{R}\right)}{A\left(b \rightarrow s \gamma_{L}\right)} \approx \frac{m_{s}}{2 E} \approx \frac{m_{s}}{m_{b}}
$$

where $E$ is the $s$ quark energy, which is roughly one half of the $b$ quark mass. This ratio is just a reflection of the fact that the gauge vector boson $(W)$ couples only to the lefthanded chirality fields, therefore in the helicity basis the $s$-quark is predominantly left-handed with a small amplitude to be right-handed given by the ratio $\mathcal{R}$. The photon has the same polarization as the $s$-quark due to conservation of the angular 
momentum.

The time-dependent $C P$ violation can only occur if $B^{0}$ and $\bar{B}^{0}$ have common final states, i.e. it should be possible to find the photon from $b \rightarrow s \gamma$ in both left and right handed helicity states. Therefore the expected time-dependent $C P$ asymmetry $S$ is

$$
S_{B^{0} \rightarrow K^{*} \gamma} \approx-2 \frac{m_{s}}{m_{b}} \sin 2 \beta \approx-0.035
$$

where the quark masses $m_{s}=110 \mathrm{MeV}$ and $m_{b}=4.65 \mathrm{GeV}$ and the $C P$ eigenvalue of the final state $\eta_{C P}=+1$ are used in this estimation. (Determination of the $C P$ eigenvalue of the final state is covered in details in the decay angular analysis, section 6.1.) This prediction does not take into account contributions to the asymmetry from $(b \rightarrow s \gamma g)$ hadronization effects, which might allow different photon polarization. This issue is discussed in the following section.

\subsubsection{Hadronic corrections}

The simple picture of the $C P$ violating asymmetries in $B^{0} \rightarrow K^{0} \pi^{0} \gamma$ requires corrections in the case of "multi-body" interaction, e.g. $b \rightarrow s \gamma g$, where $g$ stands for a gluon. An estimate of these effects using the Operator Production Expansion (OPE) method is reported in reference [23].

In the OPE framework the decay amplitude for $b \rightarrow s \gamma$ is dominated by a single 


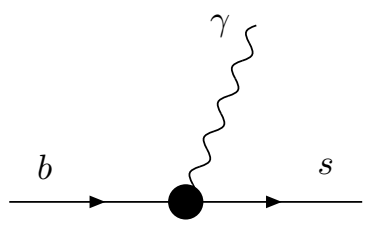

$\mathrm{O}_{7}$

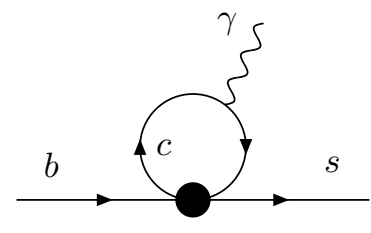

$\mathrm{O}_{2}$

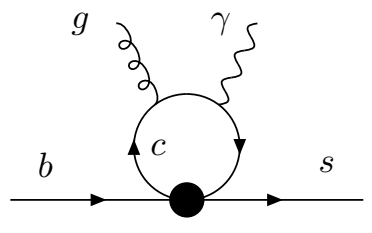

$\mathrm{O}_{2}^{\prime}$

Figure 2.8: Feynman diagrams of the dominant operators contributing to $B^{0} \rightarrow$ $K^{0} \pi^{0} \gamma$ decay amplitude. The $O_{2}^{\prime}$ represents the leading order contribution to the decay amplitude with opposite handiness

dipole-type electromagnetic operator:

$$
O_{7}=\frac{e}{16 \pi^{2}} \bar{s} \sigma^{\mu \nu} F_{\mu \nu}\left(m_{b} P_{R}+m_{s} P_{L}\right) b
$$

where $P_{R, L}=\left(1 \pm \gamma_{5}\right) / 2$. The $m_{b} P_{R}$ part of the operator contributes to the lefthanded amplitude, whereas the $m_{s} P_{L}$ part contributes to the right-handed one and we get the same estimate of the ratio of the left-handed and right-handed amplitudes: $\mathcal{R}=\frac{m_{s}}{m_{b}}$.

Neglecting the $m_{s} P_{L}$ part of $O_{7}$ it is possible to show that in the two-body limit all operators lead to the left-handed photon polarization, but in the case of multi-body interaction the other operators can contribute to the right-handed amplitude. The dominant contribution to $b \rightarrow s \gamma_{R}$ amplitude comes from the $O_{2}$ operator:

$$
O_{2}=\left(\bar{c} \gamma^{\mu} P_{L} b\right)\left(\bar{s} \gamma_{\mu} P_{L} c\right)
$$

Figure 2.8 shows the leading order operators contributing to $B^{0} \rightarrow K^{0} \pi^{0} \gamma$ decay amplitude. 
Evaluation of the operators matrix elements provides an estimation of the ratio of the right-handed and left-handed decay amplitudes, which in the case of the inclusive $B \rightarrow X_{s} \gamma$ decay is of the order of 0.1 . Unfortunately a measurement of the timedependent $C P$ violating asymmetries for inclusive decays is essentially impossible, since significant number of the final states are self-tagging and do not allow the interference of the decay with and without mixing to occur. In principle it is still possible to perform a semi-inclusive measurement when a significant number of $B \rightarrow$ $X \gamma$ with $X$ decaying to a $C P$ final state is accessible experimentally.

Calculation of the hadronic corrections of the exclusive decays involves significant long-distance effects, which are difficult to evaluate with good precision. In general it is expected that the ratio $\mathcal{R}$ for the exclusive decays is of the order of $\Lambda_{Q C D} / m_{b}$. A more detailed calculation reveals that in the case of $B^{0} \rightarrow K^{* 0} \gamma$ the ratio is enhanced by a factor $C_{2} / C_{7}$ :

$$
\mathcal{R}^{Q C D} \sim \frac{1}{3} \frac{C_{2}}{C_{7}} \frac{\Lambda_{Q C D}}{m_{b}} \sim 0.1
$$

where the "effective" Wilson coefficients at leading order are $C_{2}=1.1$ and $\left|C_{7}\right|=0.31$.

Overall it is hard to expect that an observation of $\left|S_{K_{S} \pi^{0} \gamma}\right|$ of the order of 0.1 or smaller can be attributed to new physics, whereas a measurement of a significantly larger asymmetry is a clear indication of new physics. The hadronic effects might also lead to a significant asymmetry dependence on the photon energy, which makes it harder to interpret the result of averaging over whole mass range of $K_{S} \pi^{0}$ system if the resonance structure of the decay is ignored. On the other hand this dependence 
can be used to determine the significance of the contribution of non-dipole operators. A difference in the $C P$ violating asymmetry of two resonances with identical $J^{P C}$ would also indicates a non-dipole contribution.

\subsubsection{Alternative methods of measuring the photon polariza- tion}

There are other potential methods to probe the photon polarization. One is based on the idea of using the photon conversion to an $e^{+} e^{-}$pair to measure the photon polarization directly by measuring the angle between the decay plains of $\gamma \rightarrow e^{+} e^{-}$ and $X \rightarrow K \pi$ [26]. The major experimental challenge of this approach is the small opening angle of the electron-positron pair, which in the case of the Bethe-Heitler conversion (external conversion in interaction with the detector material) is of the order of $m_{e} / E \sim 10^{-3}$, making it essentially impossible to measure the decay plain orientation. Fortunately one can use the internal photon conversion, which has significantly larger opening angle of the order of $\sqrt{m_{e} / E} \sim 0.1-0.01$. In this case we essentially reconstruct the $B^{0} \rightarrow K^{*} l^{+} l^{-}$decay with low $Q^{2}$ (invariant mass of the lepton pair). The well known pole at $Q^{2}=0$ increases the branching ratio of $B^{0} \rightarrow K^{*} e^{+} e^{-}$by factor $\sim 1 / 3$ with respect to $B^{0} \rightarrow K^{*} \mu^{+} \mu^{-}$and these events can be used to probe the photon polarization. As the $Q^{2}$ increases the contributions of other operators can become comparable with the contribution of the dipole-type operator, but at the level of $Q^{2}<0.5-1 \mathrm{GeV}$ the decay amplitude is still dominated 
by the $O_{7}$ operator.

Another method is based on the measurement of the interference of various $K \pi \pi$ resonances in $B \rightarrow K \pi \pi \gamma$ decays [27]. Using a three body decay it is possible to construct a parity-odd quantity, $\overrightarrow{p_{\gamma}} \cdot\left(\overrightarrow{p_{1}} \times \overrightarrow{p_{2}}\right)$, where $\overrightarrow{p_{1}}$ and $\overrightarrow{p_{2}}$ are two of the final hadron momenta measured in the rest frame of the $K$-resonance, which can be used to probe the photon polarization. In order to extract this information one needs to observe interference of two different resonant decays to the same final state. One of the promising decay modes is $B \rightarrow K_{1}(1400) \gamma$, proceeding through the following decay channels:

$$
\begin{array}{r}
K_{1}^{+} \rightarrow\left(\begin{array}{c}
K^{*+} \pi^{0} \\
K^{* 0} \pi^{+}
\end{array}\right) \rightarrow K^{0} \pi^{+} \pi^{0} \\
K_{1}^{0} \rightarrow\left(\begin{array}{c}
K^{*+} \pi^{-} \\
K^{* 0} \pi^{0}
\end{array}\right) \rightarrow K^{+} \pi^{-} \pi^{0} .
\end{array}
$$

Defining the angle $\theta$ to be the angle between the photon direction $\vec{p}_{\gamma}$ and the normal to the decay plane defined by $\vec{p}_{\text {slow }} \times \vec{p}_{\text {fast }}$, where $\vec{p}_{\text {slow }}$ and $\vec{p}_{\text {fast }}$ are the momenta of the slower and faster pions in the $K_{1}$ rest frame, one can probe the photon polarization from the $\theta$ distribution, which has different forms for the left-handed and right-handed photons. The integrated up-down asymmetry $(\theta \in[0, \pi / 2]$ - up, $\theta \in[\pi / 2, \pi]$ - down $)$ is estimated to be:

$$
\mathcal{A}=-(0.34 \pm 0.05) \lambda_{\gamma}
$$


where $\lambda_{\gamma}$ is the photon polarization, which in the Standard Model is approximately $+1(-1)$ for $B(\bar{B})$ decays, respectively. The major experimental challenge of this approach is the requirement to separate different kaon resonances, which is a serious challenge in the case of low statistics rare $B \rightarrow X_{s} \gamma$ decays. To understand why it is essential to study the resonance structure of the decay, one has to take into account that the $B \rightarrow K^{*}(1410) \gamma$ decay is not sensitive to the photon polarization and for the $B \rightarrow K_{2}^{*}(1430) \gamma$ the integrated up-down asymmetry is zero, which might lead to a signal dilution. Given that these resonances are quite wide and have common decay channels, the measurement of the photon polarization would require significant amount of data and a complicated Dalitz analysis in order to resolve various resonances.

\subsection{Summary}

Summarizing the discussions in previous sections one can conclude that the $B^{0} \rightarrow$ $K_{S}^{0} \pi^{0}$ and $B^{0} \rightarrow K_{S}^{0} \pi^{0} \gamma$ decays can be sensitive to physics beyond the Standard Model. In the case of the $B^{0} \rightarrow K_{S}^{0} \pi^{0}$ a statistically significant deviation of the experimental value of the $S$-term of the time-dependent $C P$ violating asymmetry from the expected Standard Model value, $0.69 \pm 0.03(\exp ) \pm 0.10$ (theory), would indicate a new physics contribution. A similar conclusion can be drawn in the case of $B^{0} \rightarrow K_{S}^{0} \pi^{0} \gamma$ if the experimental measurement of $\left|S_{K_{S} \pi^{0} \gamma}\right|$ is found to be significantly larger than 0.1. This dissertation presents the experimental measurements for both 
decays. 


\section{Chapter 3}

\section{The BABAR Detector}

\subsection{Overview}

The BABAR detector was designed as a general purpose detector with good tracking, electromagnetic calorimetry and particle identification capability to measure variety of different physics processes. The design was optimized to achieve the primary goal of the experiment: the study of the $C P$ violating effects in $B$ meson decays. There is a number of publications that cover the detector design and performance of various subdetectors in details. Among them "The BaBar Technical Design Report" (TDR) [28] and "The BaBar Detector" [29] can be used as an introduction with a broad overview of different aspects of the detector design, construction and performance evaluation. Here, a brief overview of the BaBar detector is presented, in particular the performance of the relevant sub-detectors that have the biggest impact on the measurement 


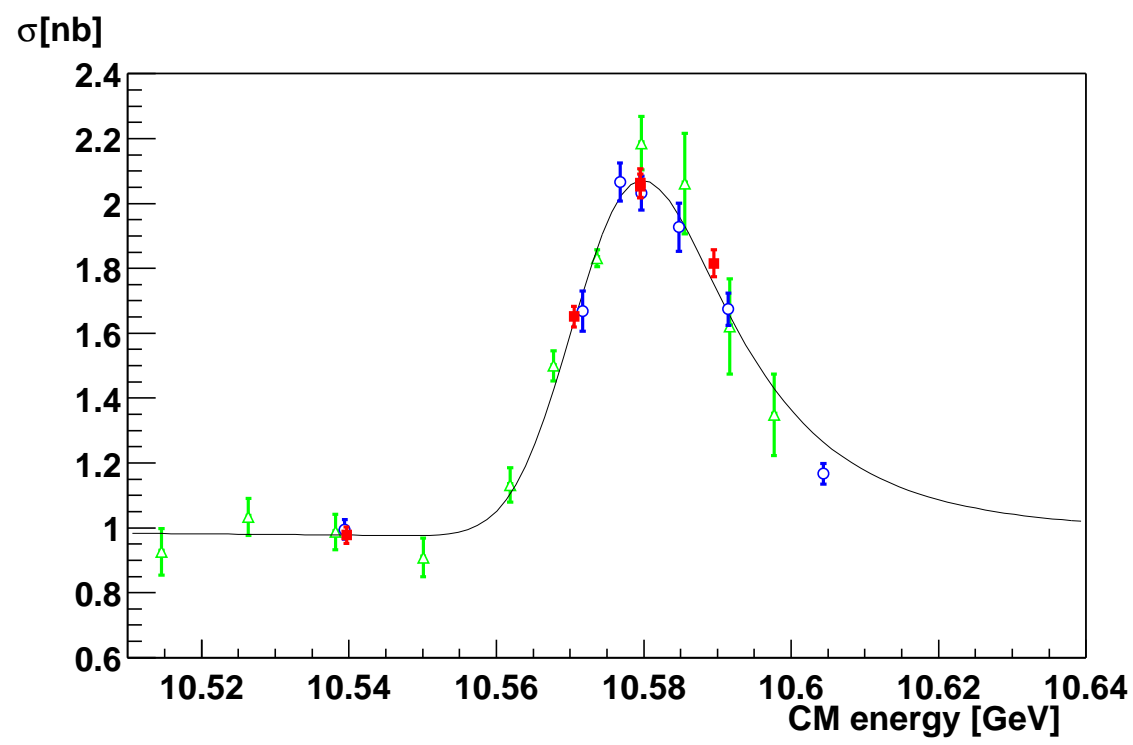

Figure 3.1: Visible cross-section of the $\Upsilon(4 S)$ resonance. The peak cross-section of $e^{+} e^{-} \rightarrow b \bar{b}$ is $1.101 \pm 0.005 \pm 0.022 \mathrm{nb}$. Continuum cross-section has been suppressed by applying event shape criteria.

of the time-dependent $C P$ violation in $B^{0} \rightarrow K_{S}^{0} \pi^{0}$ and $B^{0} \rightarrow K_{S}^{0} \pi^{0} \gamma$ decays.

The PEP-II $B$ Factory is an asymmetric electron-positron collider operating at the center of mass energies around $10.58 \mathrm{GeV}$ corresponding to the $\Upsilon(4 S)$ resonance, which in more than $96 \%$ of cases decays to a $B \bar{B}$ pair. Figure 3.1 shows the crosssection of $e^{+} e^{-}$to hadrons in the vicinity of this resonance after applying certain continuum suppressing criteria. The $\Upsilon(4 S)$ resonance with a peak cross-section of about $1.1 \mathrm{nb}$ sits on a continuum background with a cross-section of nearly $3 \mathrm{nb}$, therefore suppression of the continuum background $\left(e^{+} e^{-} \rightarrow q \bar{q}\right)$ represents a significant experimental challenge. In the case of a symmetric energy collider the $B$ mesons would be nearly at rest in the laboratory frame, since the $\Upsilon(4 S)$ resonance mass is close to the total mass of the two $B$ mesons. This would make the measurement 
of the time-dependent $C P$ violation essentially impossible. By boosting the $\Upsilon(4 S)$ frame along the beam direction, one can obtain measurable decay vertex separations between the $B$ and $\bar{B}$ mesons, which can be used to determine the time evolution of the $B \bar{B}$ system. This is achieved with an asymmetric collider with an electron beam of $9.0 \mathrm{GeV}$ and a positron beam of $3.1 \mathrm{GeV}$, resulting in a Lorentz boost of $\beta \gamma=0.56$, which leads to an average decay point separation along the beam $(\Delta z)$ of $260 \mu \mathrm{m}$.

Another reason why the asymmetric design is favorable for the time-dependent asymmetry measurements comes from the fact that the distance between decay vertices can be easily translated to the decay time difference without measuring the decay vertex of the $\Upsilon(4 S)$ resonance. Due to the boost of the center of mass frame with respect to the laboratory frame, the $B$ mesons, which are almost at rest in the center of mass frame, acquire significant momenta in the laboratory frame and both of them fly forward in the laboratory frame leaving the decay vertex of the $\Upsilon(4 S)$ behind. In the case of a symmetric collider, with no boost of the center of mass frame, it is only possible to measure the sum of decay times if the production point of the $B \bar{B}$ pair is not known. A more detailed discussion of the calculation of the decay time difference using the distance between $B$ meson vertices is presented in Chapter 4 in Section 4.1.3. Figure 3.2 shows the accelerator configuration at SLAC, including the linac, the PEP-II asymmetric $e^{+} e^{-} \mathrm{B}$ factory and the single interaction point instrumented by the BaBar detector.

Figure 3.3 shows a longitudinal section through the BABAR detector center, and 


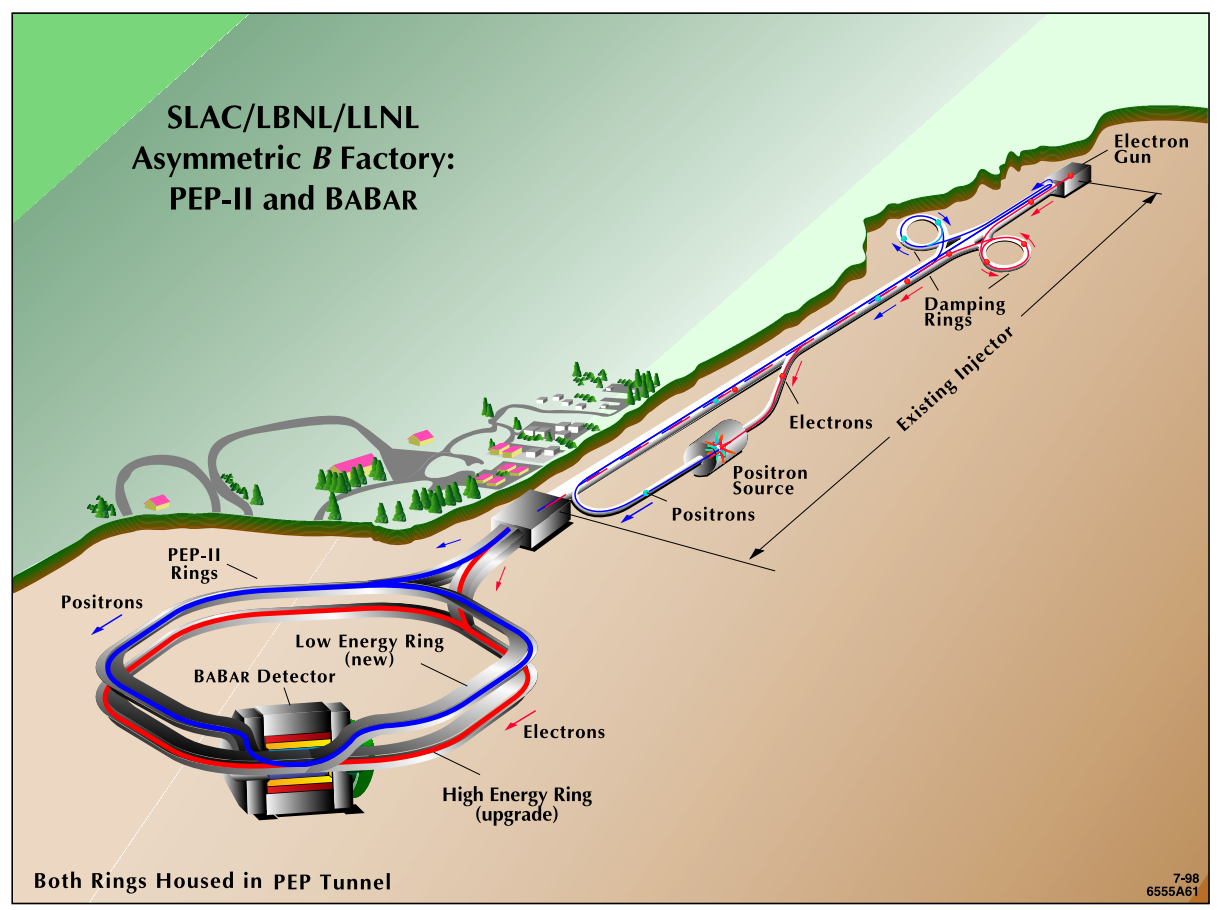

Figure 3.2: The Stanford linear accelerator with the PEP-II storage rings and the $B A B A R$ detector

Figure 3.4 shows an end view. The BABAR detector consists of five major subdetectors. The Silicon Vertex Tracker (SVT) and the Drift Chamber (DCH) make up the BABAR tracking system. The SVT is the first component of the tracking system, which provides precise measurement of the decay vertex positions and detects low momentum charged tracks. The DCH serves as the outer component of the tracking system and in addition provides the ionization energy loss per unit length $(d E / d x)$ measurements for use in particle identification (PID). The CsI(Tl) Electromagnetic calorimeter (EMC) provides measurements of energy deposit by charged and neutral particles. The EMC is critical to identification of electrons and photons as well as $K_{L}$. The other two sub-detectors: the Detector of Internally Reflected Cherenkov 


\begin{tabular}{|c|c|c|c|c|c|c|c|}
\hline System & Acceptance & Channels & $\overline{\overline{\mathrm{ADC}}}$ & $\overline{\text { TDC }}$ & $\overline{\overline{\text { Layers }}}$ & Segmentation & Performance \\
\hline$\overline{\text { SVT }}$ & $20.1^{\circ}-150.2^{\circ}$ & $\overline{150 \mathrm{~K}}$ & 4 bits & - & $\overline{5}$ & $\begin{array}{l}50-100 \mu m r-\phi \\
100-200 \mu m z\end{array}$ & \multirow{2}{*}{$\begin{array}{l}\sigma_{d_{0}}=55 \mu m \\
\sigma_{z_{0}}=65 \mu \mathrm{m} \\
\sigma_{\phi}=1 \mathrm{mrad} \\
\sigma_{\text {tand }}=0.001 \\
\sigma_{p_{t}} / p_{t}=0.47 \% \\
\sigma_{d E / d x}=7.5 \%\end{array}$} \\
\hline $\mathrm{DCH}$ & $17.2^{\circ}-152.6^{\circ}$ & 7,104 & 8 bits & $2 \mathrm{~ns}$ & 40 & $6-8 \mathrm{~mm}$ & \\
\hline DIRC & $25.5^{\circ}-141.4^{\circ}$ & 10,752 & - & $0.5 \mathrm{~ns}$ & 1 & $\begin{array}{l}35 \times 17 \mathrm{~mm}^{2} \\
(r \Delta \phi \times \Delta r) \\
144 \text { bars }\end{array}$ & $\begin{array}{l}\sigma_{\theta_{C}}=2.5 \mathrm{mrad} \\
\text { per track }\end{array}$ \\
\hline$\overline{\operatorname{EMC}(\mathrm{C})}$ & $27.1^{\circ}-140.8^{\circ}$ & $2 \times 5760$ & 18 bits & - & 1 & $\begin{array}{l}47 \times 47 \mathrm{~mm}^{2} \\
5760 \text { crystals }\end{array}$ & $\begin{array}{l}\sigma_{E} / E=3.0 \% \\
\sigma_{\phi}=3.9 \mathrm{mrad}\end{array}$ \\
\hline $\operatorname{EMC}(\mathrm{F})$ & $15.8^{\circ}-27.1^{\circ}$ & $2 \times 820$ & & & 1 & 820 crystals & $\sigma_{\theta}=3.9 \mathrm{mrad}$ \\
\hline IFR (C) & $47^{\circ}-123^{\circ}$ & $22 \mathrm{~K}+2 \mathrm{~K}$ & $1 \mathrm{bit}$ & $0.5 \mathrm{~ns}$ & $19+2$ & $20-38 \mathrm{~mm}$ & $90 \% \mu^{ \pm}$eff. \\
\hline $\operatorname{IFR}(\mathrm{F})$ & $20^{\circ}-47^{\circ}$ & $14.5 \mathrm{~K}$ & & & 18 & $28-38 \mathrm{~mm}$ & $6-8 \% \pi^{ \pm}$mis-id \\
\hline IFR (B) & $123^{\circ}-154^{\circ}$ & $14.5 \mathrm{~K}$ & & & 18 & $28-38 \mathrm{~mm}$ & $(1.5-3.0 \mathrm{GeV})$ \\
\hline
\end{tabular}

Table 3.1: Overview of the coverage, segmentation and performance of the BABAR detector systems. The notation $(\mathrm{C}),(\mathrm{F})$ and $(\mathrm{B})$ refers to the central barrel, forward and backward components of the system, respectively. The sub-detector acceptance is measured in the polar angle with the $z$-axis pointing along the high energy beam.

light (DIRC) and the Instrumented flux return (IFR) are primarily used for particle identification. The DIRC provides an efficient kaon/pion separation, whereas the IFR allows to identify muons and $K_{L}$ s. Table 3.1 summarizes resolution and acceptance parameters of all sub-detectors.

\subsection{The collider and beam parameters}

The PEP-II facility consists of two storage rings: the high energy electron ring (HER) and the low energy positron ring (LER). Since the start of the data taking in 1999 the machine has been running with coasting beams with short periods of new particle injections. At the end of RUN4 (2004) the running conditions were changed to the continuous injection mode, which increases the effective luminosity and makes the running conditions much more stable. 


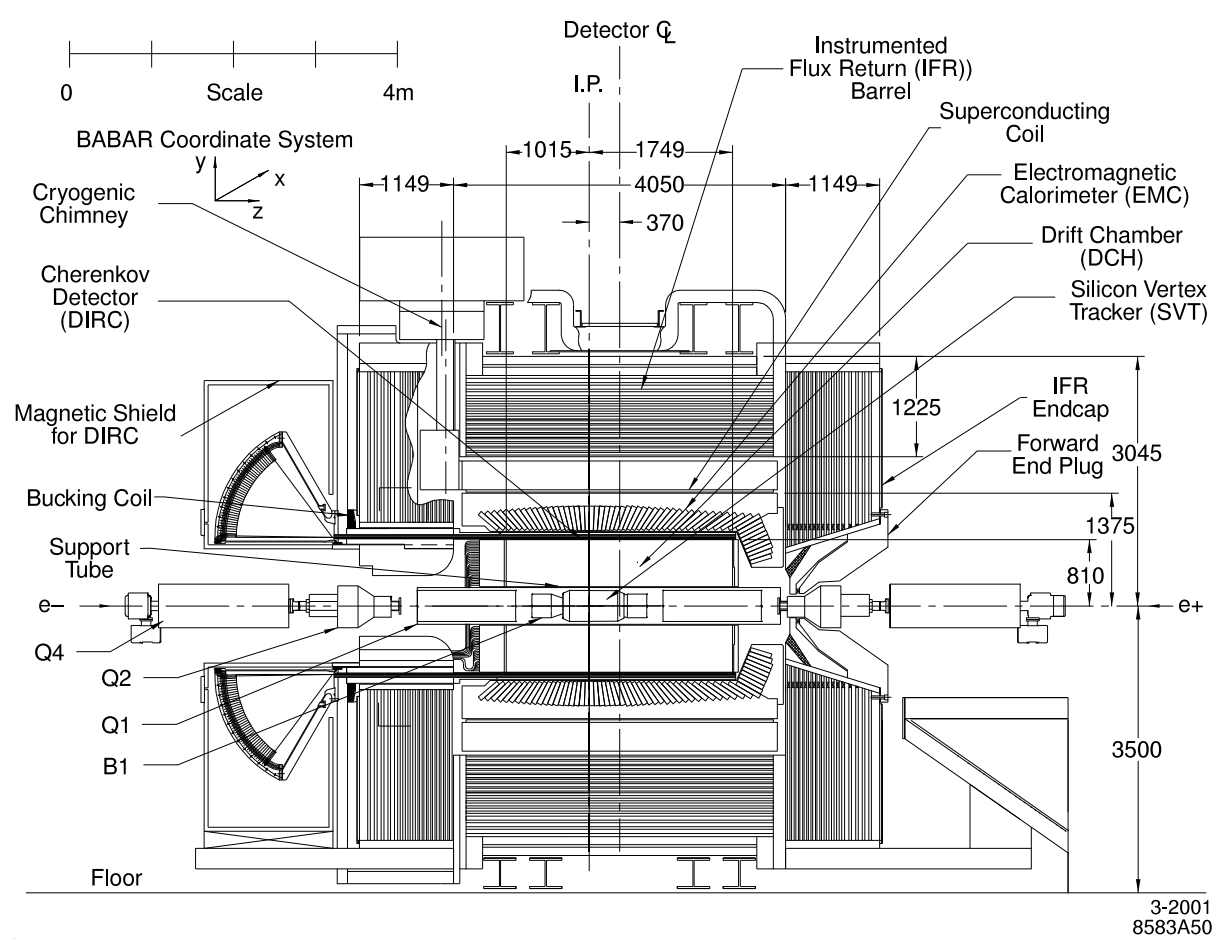

Figure 3.3: BABAR detector longitudinal section

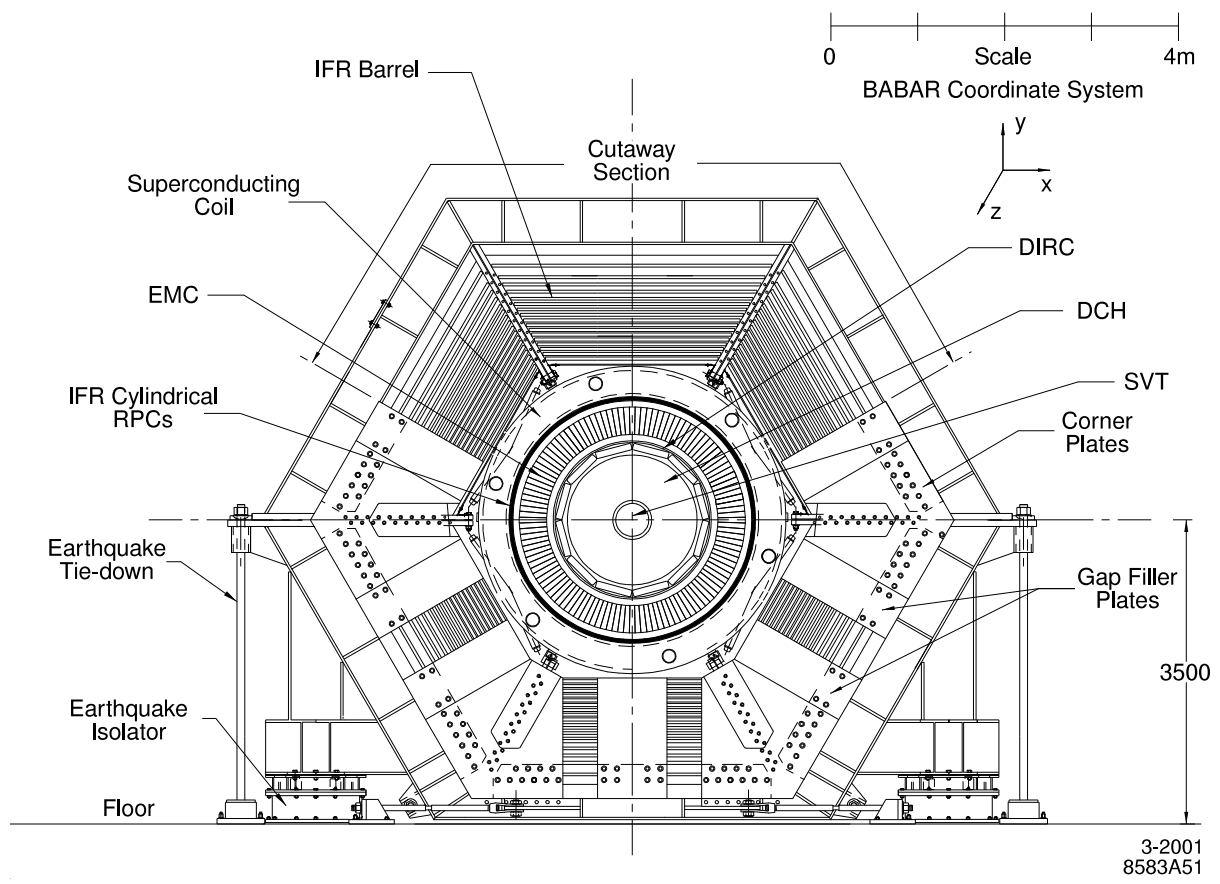

Figure 3.4: BABAR detector end view 
The beam parameters can be found in Table 3.2. The luminous region at the intersection of the two beams (the beam spot) has a typical size of $120 \times 4 \mu m^{2}$ in the $x-y$ plain and its position is constantly monitored. The small size of the beam spot and the fact that the $B$ meson typically moves only $20-30 \mu m$ in the $x-y$ plane, provides a very useful constraint on the decay vertices of the $B$ mesons in the transverse plane. This fact is exploited in the measurements of the $B^{0} \rightarrow K_{S}^{0} \pi^{0}$ and the $B^{0} \rightarrow K_{S}^{0} \pi^{0} \gamma$ decays, where the $z$-position of the $B$ meson decay vertex is essentially determined as an intersection of the beam direction and the direction of the $K_{S}$ meson.

The luminosity is measured on-line using radiative Bhabha decays and more accurately off-line using different QED processes such as $e^{+} e^{-}$and $\mu^{+} \mu^{-}$pair production. The relative rate of $B \bar{B}$ to lepton pair production is also measured on-line in order to monitor the center of mass beam energy and help maximize the $B \bar{B}$ production rate by running close to the $\Upsilon(4 S)$ peak. A typical shift of the center of mass energy of $2 \mathrm{MeV}$ leads to $2.5 \%$ change in the ratio of the number of $B \bar{B}$ hadronic events to $e^{+} e^{-} \rightarrow l^{+} l^{-}$events. Some fraction (around 10\%) of the total data taking is devoted to the off-peak running at about $40-50 \mathrm{MeV}$ below the $\Upsilon(4 S)$.

Figure 3.5 shows the total integrated luminosity recorded since the start of the experiment. The peak luminosity achieved by PEP-II is $9.2 \times 10^{33} \mathrm{~cm}^{-2} \mathrm{sec}^{-1}$. The best day, week and month integrated luminosities are $710.5 \mathrm{pb}^{-1}, 4.46 \mathrm{fb}^{-1}$ and $17.0 \mathrm{fb}^{-1}$, respectively. 


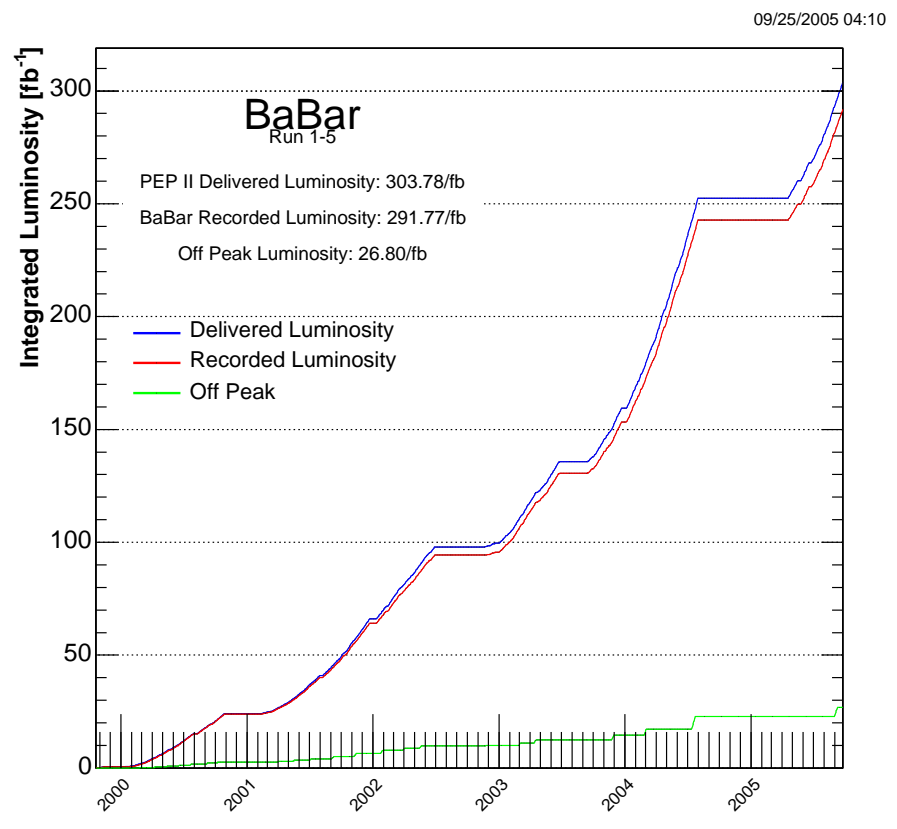

Figure 3.5: Total integrated luminosity of the BABAR detector.

Besides the continuum background ( $e^{+} e^{-} \rightarrow q \bar{q}$ interactions), a significant amount of background is associated with the machine itself. First of all the detector is exposed to the synchrotron radiation generated by bending of the beam trajectories in the vicinity of the interaction region. Other sources of backgrounds are the beam-wall and beam-gas scatterings. The latter is the primary source of radiation damage in the SVT and dominant background for the drift chamber and other sub-detectors.

To ensure safety of the detector, significant attention is paid to radiation protection and monitoring. The SVT is protected by a quick beam abort system that dumps the beams if an instantaneous radiation dose of 1 Rad is accumulated over 1 ms. Another system monitors the integrated dose over 5 minutes and if it exceeds an average level of 50-100 $\mathrm{mRad} / \mathrm{s}$ the beams are aborted. Table 3.3 summarizes 


\begin{tabular}{lccc}
\hline \hline Parameters & & \\
\hline Energy HER/LER (GeV) & & $9.0 / 3.1$ & \\
Energy spread HER/LER (MeV) & \multicolumn{3}{c}{$5.5 / 2.3$} \\
Time between collisions (ns) & 4.2 & \\
Circumference (m) & \multicolumn{3}{c}{2200} \\
Effective bend radius HER/LER (m) & \multicolumn{3}{c}{$165 / 13.75$} \\
Energy loss HER/LER (MeV/turn) & Design & Peak Luminosity & Future 2007 goal \\
\hline \hline Parameters & $750 / 2140$ & $1550 / 2450$ & $2200 / 4500$ \\
\hline Current HER/LER (mA) & 1658 & 1588 & 1715 \\
Number of bunches & 110 & 120 & 120 \\
RMS size of the luminous region $\sigma_{x}(\mu m)$ & 3.5 & 3.1 \\
RMS size of the luminous region $\sigma_{y}(\mu m)$ & 3.3 & 11 & 8.5 \\
$\beta_{y}^{*}($ mm) & $15-20$ & 9.2 & 21 \\
Luminosity $\left(10^{33} \mathrm{~cm}^{-2} \mathrm{~s}^{-1}\right)$ & 3.0 & 710 & 1300 \\
Integrated luminosity $\left(\mathrm{pb}^{-1} /\right.$ day) & 130 & & \\
\hline \hline
\end{tabular}

Table 3.2: PEP-II beam parameters

expected radiation dose and tolerance level for different sub-detectors.

With the increase of the luminosity to the level of $21 \times 10^{33} \mathrm{~cm}^{-2} \mathrm{~s}^{-1}$ planned for 2007 , it is expected that the occupancy of all sub-systems except IFR/LST reach levels that might affect resolution and reconstruction efficiency. Some work is being done to minimize this negative effect on physics performance of the experiment.

\section{$3.3 \quad$ SVT}

The silicon vertex tracker (SVT) is designed to serve as the primary device in measuring the $B$ meson decay vertex positions with adequate resolution to study timedependent $C P$ violation in $B$ decays. It consists of five layers of double-sided silicon strip sensors (Figure 3.6). The first 3 layers are placed close to the beam pipe and provide impact parameter measurements of charged tracks with high resolution. The outer layers are placed closer to the DCH to improve the SVT and DCH track 


\begin{tabular}{|c|c|c|c|}
\hline$\overline{\text { Sub-system }}$ & Limiting factor and impact & $\begin{array}{l}\text { Operational } \\
\text { limit }\end{array}$ & $\begin{array}{l}\text { First-year } \\
\text { typical }\end{array}$ \\
\hline SVT sensors and electronics & $\begin{array}{l}\text { Integrated dose; radiation } \\
\text { damage }\end{array}$ & $2 \mathrm{MRad}$ & $0.33 \mathrm{MRad}$ \\
\hline SVT sensors & $\begin{array}{l}\text { Instantaneous dose; diode } \\
\text { short }\end{array}$ & $1 \mathrm{Rad} / \mathrm{ms}$ & \\
\hline DCH electronics & $\begin{array}{l}\text { Integrated dose; radiation } \\
\text { damage }\end{array}$ & $20 \mathrm{kRad}$ & $100 \mathrm{Rad}$ \\
\hline DCH wire current & $\begin{array}{l}\text { Accumulated charge; wire } \\
\text { aging }\end{array}$ & $100 \mathrm{mC} / \mathrm{cm}$ & $8 \mathrm{mC} / \mathrm{cm}$ \\
\hline DCH total current & HV system limitations & $1000 \mu A$ & $250 \mu A$ \\
\hline DIRC PMTs & $\begin{array}{l}\text { Counting rate; TDC dead- } \\
\text { time }\end{array}$ & $200 \mathrm{kHz}$ & $110 \mathrm{kHz}$ \\
\hline EMC crystals & $\begin{array}{l}\text { Integrated dose; radiation } \\
\text { damage }\end{array}$ & $10 \mathrm{kRad}$ & $0.25 \mathrm{kRad}$ \\
\hline
\end{tabular}

Table 3.3: The detector radiation tolerance.

segments matching in order to reconstruct charged tracks transversing both subdetectors. The $\phi$ strips on one side of the sensors run parallel to the beam direction and the $z$ strips on the other side are oriented transversely to the beam axis. Hit position resolution for the inner layers is about 10-15 $\mu \mathrm{m}$ and $40 \mu \mathrm{m}$ for the outer layers.

The SVT also provides precise measurements of the track directions, which plays an essential role in particle identification for high momentum tracks. The measurement of the Cherenkov angle in the DIRC depends on precise information on the track directions at the entrance to the DIRC.

Another important role of the SVT is detection of low momentum tracks with transverse momenta less than $120 \mathrm{MeV}$ - the minimum that can effectively be reconstructed in the DCH alone. This is essential for reconstruction of the slow pions 


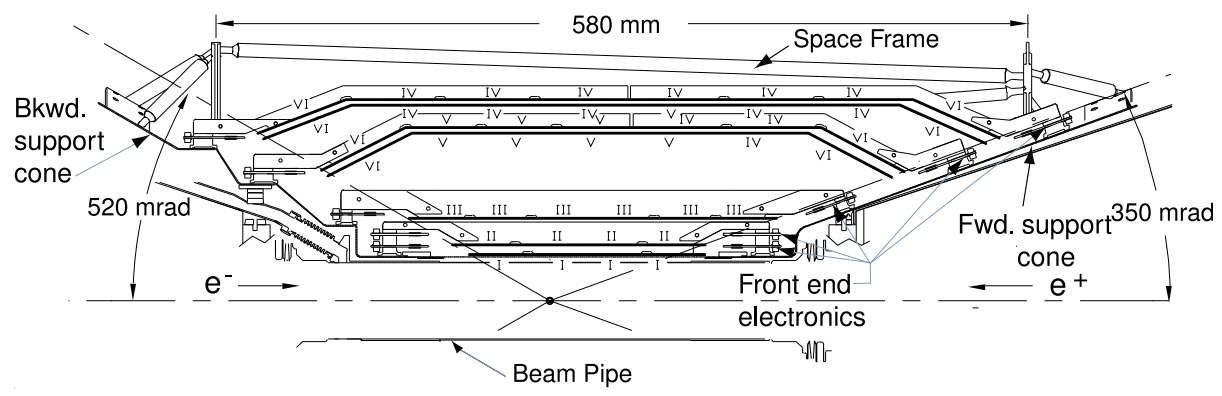

Figure 3.6: Side-view of the SVT.

from $D^{*}$ meson decays. The SVT can effectively reconstruct tracks with transverse momenta in the range $50-120 \mathrm{MeV}$.

The SVT is placed inside the support tube, which is mounted on the PEP-II accelerator supports. This allows for movement between SVT and the rest of the detector. The relative position of SVT with respect to DCH is constantly monitored and the global SVT alignment is performed as part of the rolling calibration during the event reconstruction. Besides the global alignment of the SVT, a local alignment of the SVT sensors is performed. The local alignment is quite stable over time, but it can change after magnet quenches or detector access.

The SVT can also provide up to ten measurements of $d E / d x$ per track. Removing the fraction of clusters with highest and lowest $d E / d x$ measurements, it is possible to achieve a $2 \sigma$ separation between kaons and pions with momenta up to $500 \mathrm{MeV}$. 


\subsection{The Drift Chamber}

The main function of the drift chamber $(\mathrm{DCH})$ is the efficient detection of charged particles and measurement of their momenta. It consists of 40 layers of hexagonal cells made of field shaping and sense wires. In order to measure the longitudinal position of the tracks, 24 layers are placed at a small angle with respect to the $z$-axis. The low amount of material inside the DCH helps to reduce the effect of multiple Coulomb scattering and makes it possible to achieve good momentum and direction resolution.

The drift chamber volume is filled with 80:20 mixture of helium:isobutane at a constant overpressure of 4 mbar, which has a radiation length that is five times larger than typically used argon based mixtures. During normal operation, one full volume of fresh gas is added every 36 hours. The water concentration is kept relatively high at $3500 \mathrm{ppm}$ in order to prevent the Malter effect. This effect is the result of thin insulating deposit formations on the field wires, leading to the accumulation of a positively charged ion layer, which causes high electric fields resulting in the emission of electrons from the wire surfaces. This might lead to a continuous discharge even when no ionizing particle is present.

The drift chamber operates at $1930 \mathrm{~V}$ in the proportional mode, when the collected charge on the sense wires is proportional to the original ionization caused by a charged track. A typical gas gain is of the order of $10^{5}$ and it is monitored by a dedicated gas gain chamber. 


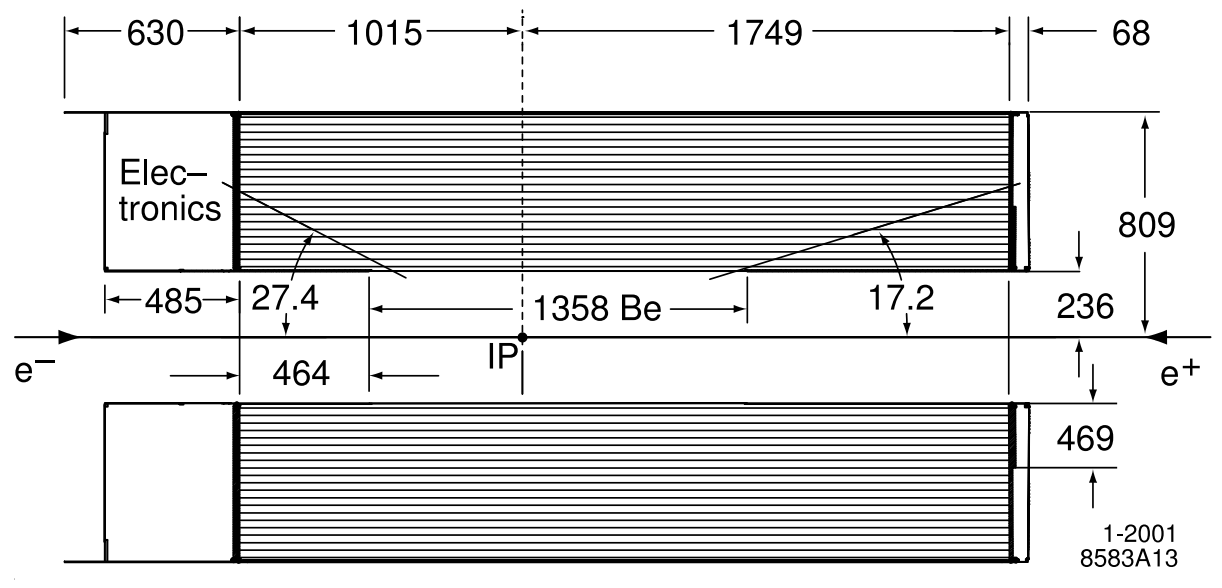

Figure 3.7: Longitudinal section of the DCH

The measurements of the ionization energy loss $(d E / d x)$ in the DCH have a typical resolution of about $7 \%$, which allows for unambiguous separation of pions and kaons with momenta up to $700 \mathrm{MeV}$ (Figure 3.8). Various effects can influence the $d E / d x$ measurements, such as changes in the gas pressure and temperature, imperfection of the cell geometry and charge collection, signal saturation due to space charge build up and non-linearity in the expected energy loss at large dip angles. The $d E / d x$ values are corrected for these effects in a dedicated calibration procedure.

The DCH track reconstruction efficiency in the acceptance region is typically about 93-94\%. It is estimated as the efficiency to find a track in the DCH if it was detected in the SVT. The efficiency estimation errors are dominated by the uncertainty in the correction for the fake tracks in the SVT. 


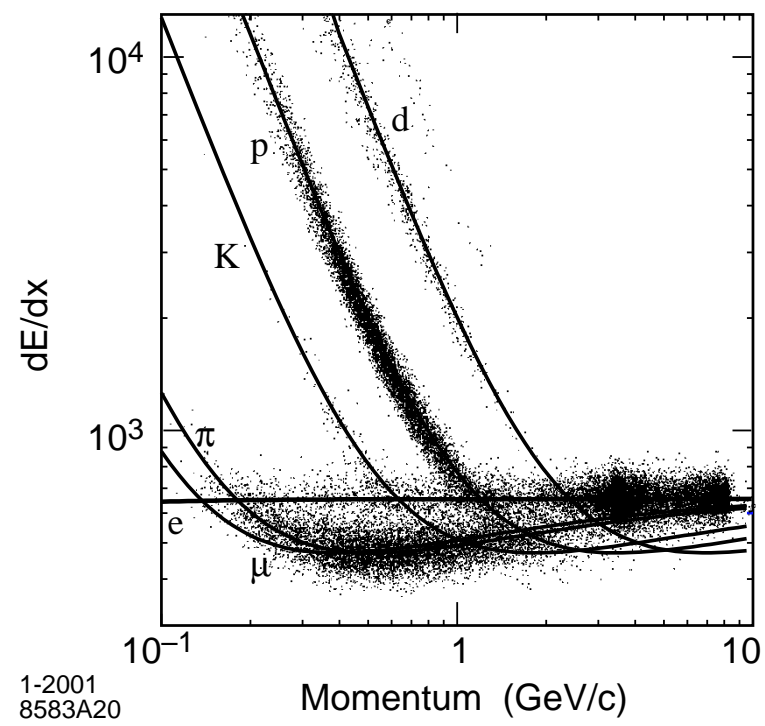

Figure 3.8: $d E / d x$ in the DCH as a function of track momenta for different charged particles.

\subsection{The EMC}

The electro-magnetic calorimeter (EMC) is dedicated to the detection of photons and measurements of energy deposition of charged and neutral particles. Only photons and electrons deposit most of their energy in the EMC. This allows efficient discrimination of electrons and positrons from other charged tracks, which helps to identify the $B$ meson flavor in the semi-leptonic decays. The ability to measure the $B$ meson flavor plays an important role in measurements of time-dependent $C P$ violating asymmetries.

The EMC consists of two parts: the barrel detector made of 5760 crystals, which covers the polar angle range from $27^{\circ}$ to $141^{\circ}$ and the forward end-cap with 820 crystals, which covers the angular range of $16^{\circ}$ to $27^{\circ}$ (Figure 3.9). The EMC crystals are thallium-doped cesium iodide $(\mathrm{CsI}(\mathrm{Tl}))$ crystals with $47 \times 47 \mathrm{~mm}^{2}$ typical front 
face area. The length of the crystals varies from $29.6 \mathrm{~cm}$ in the backward to $32.4 \mathrm{~cm}$ in the forward direction to minimize the effect of shower leakage for higher momentum particles. This length corresponds to 16-17 radiation lengths and allows for small shower leakage and good energy resolution.

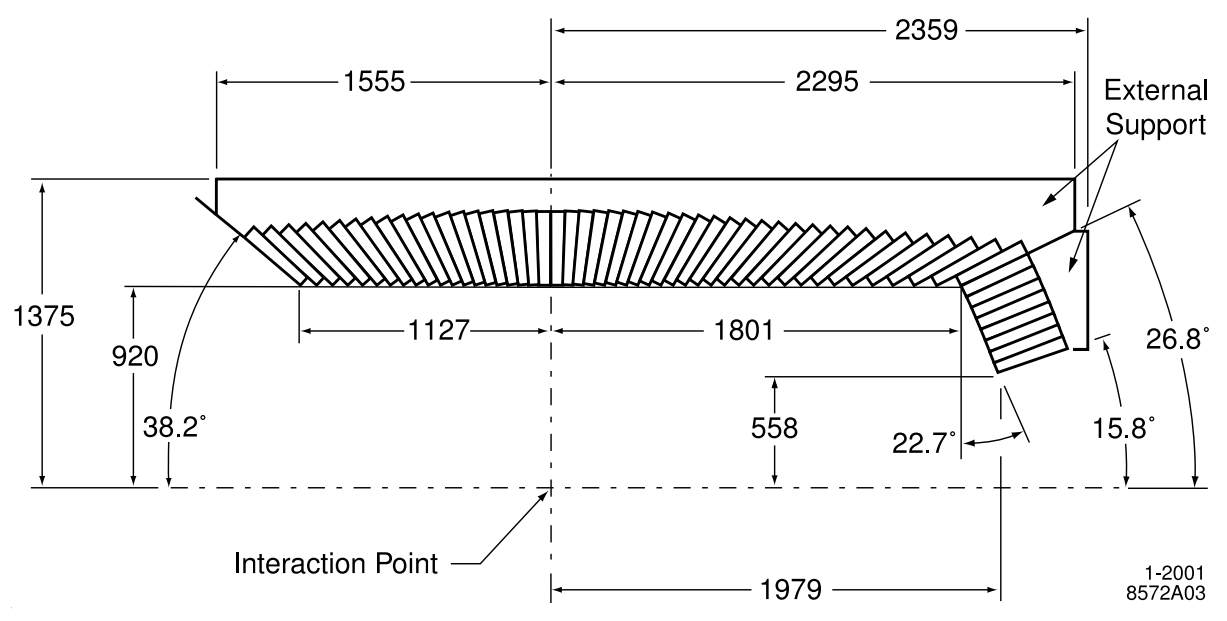

Figure 3.9: Longitudinal cross section of the EMC.

A single photon energy resolution can be expressed as:

$$
\frac{\sigma_{E}}{E}=\frac{2.3 \%}{\sqrt[4]{E(\mathrm{GeV})}} \oplus 1.9 \%
$$

where the two terms should be added in quadrature. The first term corresponds to the fluctuations in photon statistics, electronics noise and low energy beam-generated backgrounds. The second term arises from non-uniformity in the light collection, leakage or absorption in the material between and in front of the crystals. The barrel and the outer five rings of the end-cap have about 0.3-0.6 radiation length of material in front of the crystals of which 0.2 corresponds to the DIRC material. 
The $\pi^{0} \rightarrow \gamma \gamma$ decays are reconstructed from combinations of photon clusters in the EMC. The typical $\pi^{0}$ reconstruction efficiency is of the order of $50-60 \%$ including the geometrical acceptance. The $\pi^{0}$ mass resolution is dominated by the energy resolution for $\pi^{0}$ energies up to $2 \mathrm{GeV}$ and the angular resolution of the clusters at higher energies. Above $3 \mathrm{GeV}$, a significant number of $\pi^{0} \mathrm{~s}$ cannot be reconstructed as two photon clusters or bumps, as the showers of the two photons are essentially merged. In this case it is still possible to identify $\pi^{0} \mathrm{~s}$, albeit with lower efficiency, by using information on the lateral distribution of the energy deposition.

The crystal light yield may differ from one crystal to another and it varies with time due to radiation damage. The EMC energy response is calibrated at two different energy ranges. At the low end a flux of low-energy neutrons is used to irradiate Fluorinert to produce photons of $6.13 \mathrm{MeV}$. At the higher end (3-9 GeV) Bhabha events are used for energy calibration.

The crystal size $\left(47 \times 47 \mathrm{~mm}^{2}\right)$ is large compared to the required angular resolution, but the position of the center of an energy deposition can be measured with much better resolution than the size of the crystals. Since a typical photon shower is shared between about 10 crystals, by using energy sharing between crystals, one can locate the photon position with precision of about $1 \mathrm{~mm}$, which corresponds to a few mrad angular resolution. If the showers of two particles overlap it is possible to reconstruct them separately by fitting for the expected shower size and particle energies. 


\subsection{DIRC}

The Detector of Internally Reflected Cherenkov light (DIRC) is a new kind of Cherenkov detector with exceptional particle identification capabilities, which allows for effective background suppression and increased sensitivity in many interesting physics analyses. Good hadron identification is essential for $B$ flavor tagging in time-dependent $C P$ violation measurements. The EMC, together with $d E / d x$ measurements in $\mathrm{DCH}$ and the Cerenkov angle from DIRC, provides good electron identification, whereas the IFR identifies muons and the DIRC separates the other charged particles.

The DIRC allows to determine a charged particle velocity by measuring the Cherenkov angle in fused silica with a mean index of refraction $n$ of 1.4723. The Cherenkov angle $\theta_{C}$ relates to the particle velocity as:

$$
\cos \theta_{C}=\frac{1}{n \beta}=\frac{c}{n v} .
$$

Figure 3.10 shows the DIRC layout. In the detector acceptance region, the DIRC consists of 144 silica bars, which are $17 \mathrm{~mm}$ thick, $35 \mathrm{~mm}$ wide and $4.9 \mathrm{~m}$ long. When a charged track traverses the bar, the Cherenkov light through internal reflection propagates to the ends. One end of the bar has a mirror that reflects the light toward the other end, which has a window to the standoff box, where the light is detected by Photo Multiplier Tubes (PMT). The internal reflection in the bars preserves the reflection angles, allowing for the direction of the Cherenkov light to be measured. 


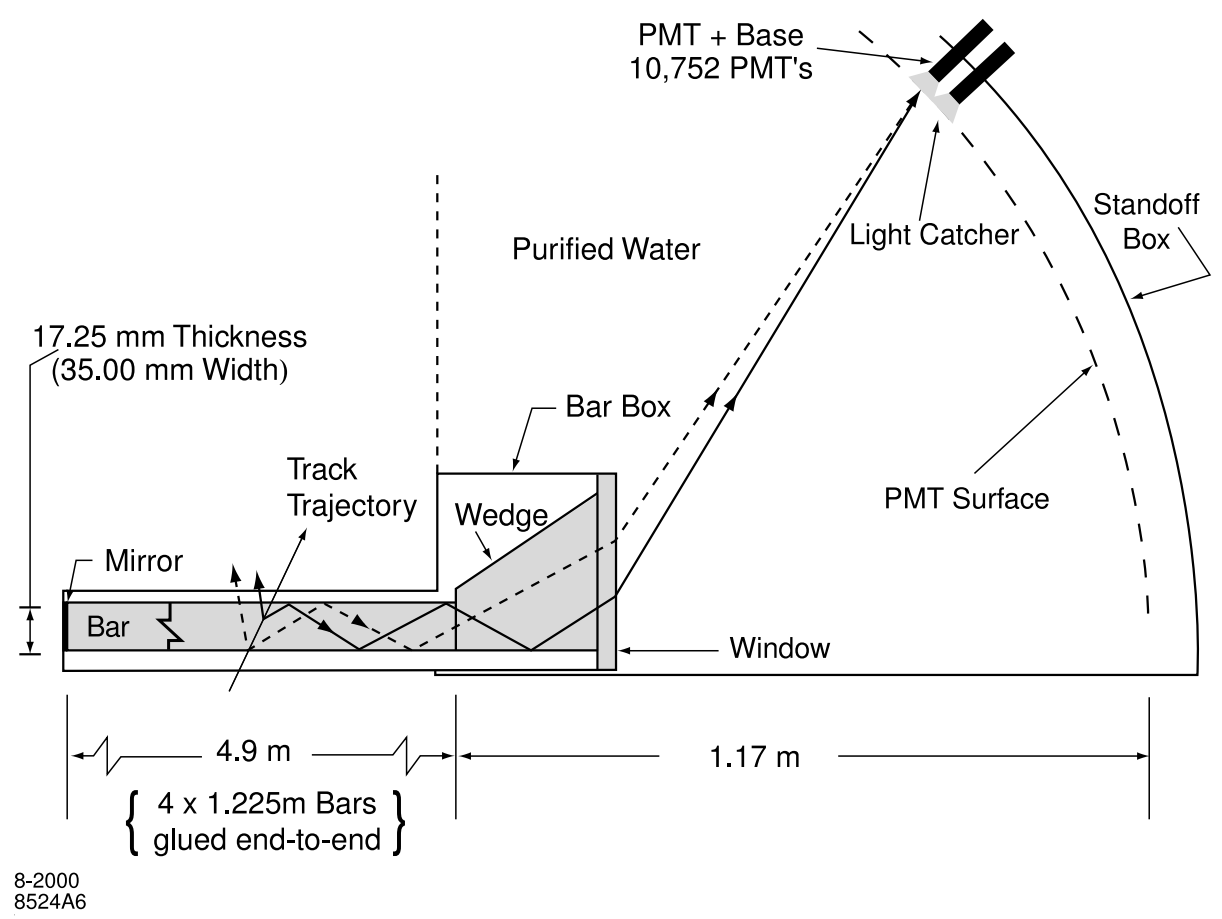

Figure 3.10: The DIRC layout.

This layout makes it possible to minimize the amount of material in front of the calorimeter, which can degrade the EMC performance. Another significant advantage of such a design is that the standoff box is placed outside the magnet flux return, where the residual field can be decreased to about 1 Gauss, whereas the nominal field inside the detector is about 15 kGauss. This allows to use conventional PMTs to collect the Cherenkov light.

Due to good angular resolution of the DIRC it is possible to achieve $\sim 4 \sigma$ or better $\pi / K$ separation for most particles of momenta higher than the DIRC threshold. Figure 3.11 shows a typical performance of the DIRC. The high momentum performance of the DIRC, in particular, plays a critical role in identification of two-body charmless decays such as $B \rightarrow \pi \pi$ and $B \rightarrow K \pi$. 


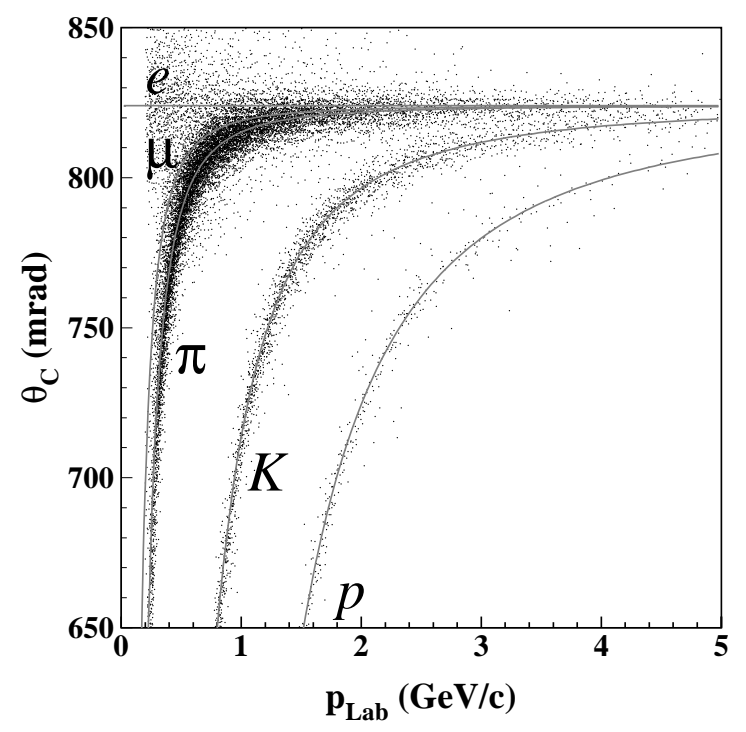

Figure 3.11: The DIRC particle identification performance. The $\theta_{C}$ is the measured Cherenkov angle and $p_{L A B}$ is the charged track momentum.

\subsection{IFR}

The Instrumented Flux Return (IFR) largely serves as the muon and $K_{L}$ identification system in the BABAR detector. Good performance of this sub-detector is essential for particle identification, because the muon mass is close to the pion mass, which makes tracks from pions and muons practically indistinguishable if only the ionization energy loss from the DCH and the Cherenkov angle from the DIRC are available.

The IFR uses the steel flux return of the magnet as a muon filter and hadron absorber. The resistive plate chambers (RPC) are used as the particle detectors in the system. The RPC is a gas detector working at higher voltage than a typical proportional chamber, which leads to a higher gas gain and formation of a streamer with the collected charge no longer proportional to the original ionization. The high 

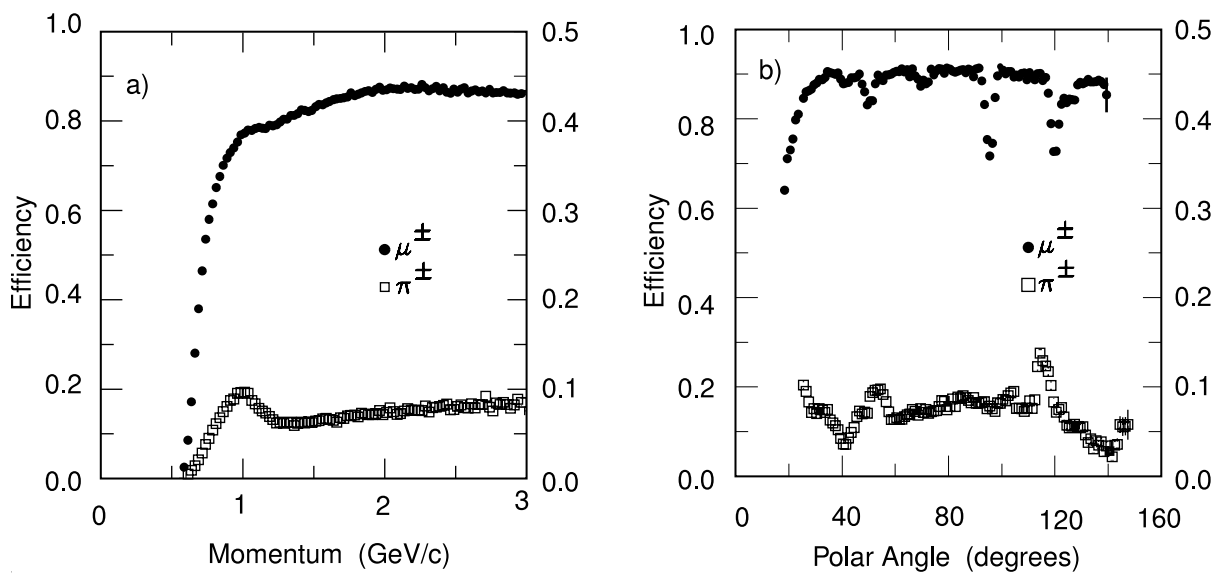

Figure 3.12: Muon efficiency (left scale) and pion misidentification probability (right scale) as a function of a) the laboratory track momentum and b) the polar angle (for $1.5<p<3.0 \mathrm{GeV}$ momentum), obtained with loose selection criteria.

resistivity of bakelite layers prevents spread of a streamer.

The RPCs layers are installed in the gaps between steel segments. The thickness of each segment was determined by a Monte Carlo study of charged and neutral hadron interaction and muon penetration. The IFR consists of 19 RPC layers in the barrel, 18 layers in the end-caps and 2 cylindrical RPC layers installed in between the EMC and the magnet coil.

Figure 3.12 shows the IFR performance for muon/pion separation. The muon detection efficiency in the first year of running was close to $90 \%$ with the pion misidentification rate of about $5-6 \%$ for the momentum range of $2.0-4.0 \mathrm{GeV}$. Significantly lower pion misidentification can be achieved for tighter criteria, but at the expense of detection efficiency.

At the early stage of the experiment it was found that a large number of RPCs were degraded in the their response and continued to degrade with time, which caused 

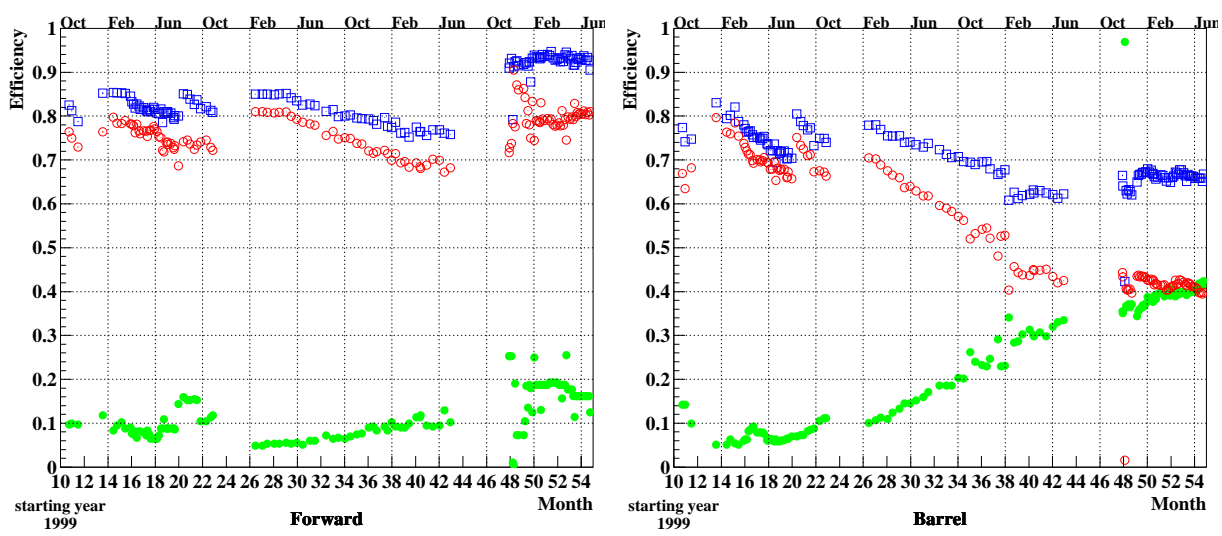

Figure 3.13: The RPC efficiency degradation with time for the forward endcap (left) and for the barrel (right) using $e^{+} e^{-} \rightarrow \mu^{+} \mu^{-}$events. Red is the average efficiency over all modules in a group, blue is the average efficiency for modules that have efficiency greater than $10 \%$ and green is the fraction of modules with efficiency less than $10 \%$.

significant degradation of the IFR performance. Figure 3.13 shows the time evolution of the RPC efficiency, showing a significant drop in performance with time. The forward end-cap RPCs were replaced with an upgraded version of RPCs in Summer of 2002. The collaboration is in the process of upgrading the system, including the barrel IFR, by replacing RPCs with Limited Stream Tubes (LST). At this point two sextants have been upgraded, with the remaining to be completed in summer 2006 .

\subsection{Trigger}

The BABAR trigger is a two-level system of a hardware level (L1) and a software level (L3). Historically, a hardware level 2 trigger was also envisioned but never implemented. It is required to be able to take physics interesting data efficiently without introducing significant dead time and be able to handle and process all logged 
data off-line with given computing resources.

The L1 trigger decision is primarily based on presence of a charged track in the DCH or significant energy deposition in the EMC. The IFR is also part of the L1 trigger, which is used for triggering on $\mu^{+} \mu^{-}$and cosmic rays. The Global Level 1 Trigger (GLT) combines inputs from different Level 1 trigger lines according to a trigger mask and issues L1Accept if the conditions are satisfied.

The Drift Chamber Level 1 trigger performs a simple charged track reconstruction in X-Y plain. At first, local track segments are found. These segments are linked into complete tracks and the corresponding transverse momenta are evaluated. In order to decrease the load on the data acquisition and the L3 trigger at high luminosity, a new DCH L1 trigger (DCZ) was designed and installed. This new trigger allows to estimate the origin of a charged track along the $z$-axis and efficiently suppress beam-generated background close to the IP.

The EMC Level 1 trigger is based on the amount of energy deposited for a group of crystals, called a tower. A typical tower is $8 \times 3(\theta \times \phi)$ array of crystals. For each tower, all crystal energies above a threshold of $20 \mathrm{MeV}$ are summed and this information is used in the trigger decision.

For a typical L1 trigger rate of $1 \mathrm{kHz}$ without using DCZ, Bhabha and physics collision events contribute $\sim 130 \mathrm{~Hz}$. The cosmic rays and random beam crossing triggers contribute $\sim 100 \mathrm{~Hz}$ and $\sim 20 \mathrm{~Hz}$, respectively. The remaining triggers are due to lost particles interacting with the detector material and the beam pipe. A 
significant amount of the beam-generated background is coming from regions 10-60 cm away from the interaction point along the beam pipe. The DCZ allows to suppress this background by using the estimated 3-D impact parameter of the charged tracks.

The Level 3 trigger performs more accurate track reconstruction, by determining the five helix track parameters for tracks with $p_{t}$ above $250 \mathrm{MeV}$. Well selected Bhabha events are pre-scaled and only a small fraction of these events is stored. The EMC energy deposition is more accurately reconstructed as well and energy clusters are determined.

Based on the Level 3 tracks and clusters, event classification is performed and the logging decision is made. The DCH filters select events with one high $p_{t}$ track or two intermediate momentum tracks originating from the IP. Two calorimeter cluster filters select events with either high energy deposits or high cluster multiplicity, requiring some minimal energy for each cluster and event mass greater than $1.5 \mathrm{GeV}$. A typical composition of the L3 output is shown in Table 3.4.

\begin{tabular}{lr}
\hline \hline Event type & Rate $(\mathrm{Hz})$ \\
\hline Hadrons, $\tau \tau$ and $\mu \mu$ & 16 \\
Other QED, 2-photon events & 13 \\
Unidentified Bhabha backgrounds & 18 \\
Beam-induced backgrounds & 26 \\
\hline Total physics accept & $\mathbf{7 3}$ \\
\hline \hline Calibration Bhabhas $\left(e^{+} e^{-}\right)$ & 30 \\
$\gamma \gamma$, Radiative Bhabhas $\left(e^{+} e^{-} \gamma\right)$ & 10 \\
Random triggers and cosmic rays & 2 \\
L1, L3 pass through diagnostics & 7 \\
\hline Total calibration/diagnostics & $\mathbf{4 9}$ \\
\hline \hline
\end{tabular}

Table 3.4: Composition of the L3 output at a luminosity of $2.6 \times 10^{33} \mathrm{~cm}^{-2} \mathrm{~s}^{-1}$. 


\subsection{Computing}

The high luminosity of the PEP-II machine and the enormous amount of data collected by $B A B A R$ represent a significant challenge for the BABAR computing. Each year the BABAR experiment accumulates a few hundred terabytes of new data. In 2002 the BABAR database reached 500 TBytes in size, which made it the world's largest database. Processing of such amount of data requires new innovating hardware and software solutions.

The logged data is processed in few stages. The first stage is called Prompt Calibration (PC). At this stage the rolling calibration is performed and its output in a form of calibration constants stored in the condition database. The data is then transfered to one of the Tier-A sites, at SLAC and in Europe, which performs the Event Reconstruction (ER). The reconstructed events are transfered back to SLAC and other sites. After a few months of data taking, when the detector performance and running conditions are stabilized and a new local SVT calibration is performed, the data is reprocessed one more time. All of these operations generate a typical daily transfer rate of 500-1000 GB/day between Europe and the USA.

In order to process such a large amount of data efficiently the event store should be able to not only handle the data, but also provide a way to persist many $\mathrm{C}++$ objects, which is necessary for handling complicated data structures with high level of abstraction. Originally for this purpose a commercial object oriented database was used, but as the amount of collected data increased, it became necessary to develop 
a new system. At that point a new ROOT based persistence technology became available, so a new event store based on a relational database and ROOT I/O was designed and successfully implemented. The new system doesn't have many of the limitations of the original event store and is expected to be able to efficiently store data for the lifetime of the experiment.

The reconstructed data has a typical size of a few hundred terabytes and normally it takes a few months to run over all events using about 50-100 CPUs simultaneously. In order to decrease the amount of data that is necessary to process for a physics analysis and to minimize the computing resource utilization, a special centralized data processing (called skimming) is used. Any user can provide an event filter, which selects events of interest for an analysis with very loose selection criteria. This allows for substantial decrease of the data volume that an end user (analyst) has to process for a given physics analysis. A typical user collection of selected events represents about $0.1-1 \%$ of all events and normally these events are copied to a new event container, which improves input/output performance of the system.

The output of the user processing is normally a ROOT file (called ntuple) that contains only analysis specific information with a typical data volume of a few gigabytes. It allows efficient interactive access to data in a data analysis software such as ROOT or PAW. Currently a new software package (called Kanga) is being developed, which uses the event store data directly in a special build of ROOT. It helps to avoid the intermediate "ntuples" and provides access to all detector information in an event 
without duplicating this information.

Many physics analyses use maximum likelihood fits to optimally extract physics information from the data. These fits and validation tools are quite complicated and most of them are based on RooFit package [30], which provides well designed framework for making and using different types of fits.

\subsection{Monte Carlo simulations}

Monte Carlo simulations represent an essential part of any physics analysis. Many exclusive and inclusive $B$ meson decays are simulated with significant amount of statistics, which allows for detailed studies of the expected detector response for different decays of interest.

$B A B A R$ was the first large experiment to use the Geant 4 software package for event simulation. A detailed detector geometry and the detector material model are used. Geant4 is responsible for simulation of electromagnetic and hadronic processes such as photo-electric effect, Compton scattering, pair production, ionization, multiple scattering etc. [31]. After physics simulation events are digitized, a sample of real background events is added. Then the standard event reconstruction is performed and the output is saved in the event store. The background samples are selected for specific data taking conditions.

A typical event simulation takes few seconds of a typical CPU time, which allows to produce large samples of simulated events using computing resources distributed over 
many computing centers. The generic $B \bar{B}$ Monte Carlo event sample is typically $3-4$ times larger the number of $B \bar{B}$ events collected by the $B A B A R$ detector. In addition to this sample, a large sample of continuum $e^{+} e^{-} \rightarrow q \bar{q}$ events is produced as well as many dedicated signal Monte Carlo samples.

The detector model and the material distribution are carefully validated in many revisions of Monte Carlo simulations. The comparison of reconstructed events from data and Monte Carlo allows to tune the model and improve understanding of the detector response. The result of such tests indicates that the tracking, energy scale, shower containment and shower development are well-understood for electromagnetic processes.

Figure 3.14 shows Monte Carlo and data distributions for the $K_{S}$ and $\pi^{0}$ masses. The Monte Carlo simulations of $\pi^{0} \mathrm{~s}$ are corrected for the energy value, resolution and reconstruction efficiency. A typical efficiency correction for $\pi^{0}$ reconstruction is of the order of a few percents with the systematic error of $3 \%$. For the charged tracks originating from the beam spot, the flat efficiency corrections are $0.25-0.5 \%$, whereas for the $K_{S}$ mesons the efficiency correction can be up to $3-5 \%$. 

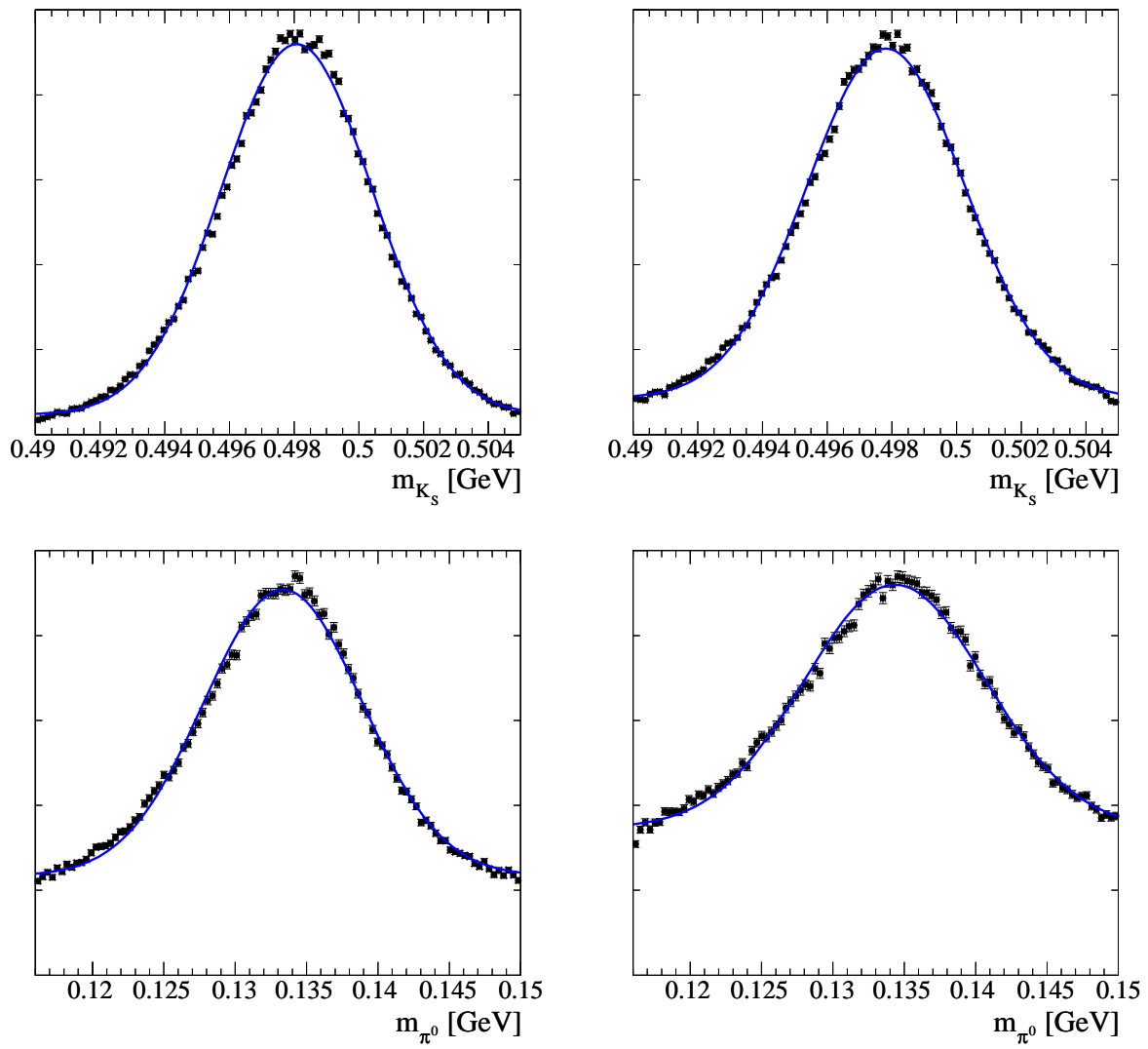

Figure 3.14: Monte Carlo (left) and data (right) mass distributions for the $K_{S}$ (top) and $\pi^{0}$ (bottom) mesons. The $K_{S}$ mass distributions fitted to Gaussian have almost identical values for Monte Carlo and data $\left(\sigma_{\mathrm{MC}}=2.3 \mathrm{MeV}\right.$ and $\left.\sigma_{\text {data }}=2.4 \mathrm{MeV}\right)$. For the $\pi^{0}$ distributions the difference is somewhat larger $\left(\sigma_{\mathrm{MC}}=5.6 \mathrm{MeV}\right.$ and $\sigma_{\text {data }}=$ $6.4 \mathrm{MeV})$ 


\section{Chapter 4}

\section{Experimental aspects of the}

\section{physics analyses}

\subsection{Measurement of the time-dependent $C P$ asym- metries}

In the theory chapter, the concept of the time-dependent $C P$ violation was presented. Here the discussion is focused on experimental aspects of such measurements. Many effects, such as the $B$ meson flavor tagging efficiency, tagging misidentification or an asymmetry in the tagging efficiency, can influence the $C P$ violating asymmetry measurements.

The time evolution equation 2.14, presented in the theory chapter, corresponds to the case of perfect $B$ flavor tagging with no asymmetry in the tagging of the $B^{0}$ and 
$\bar{B}^{0}$ mesons. Taking into account these effects, the time evolution of the $B^{0}$ and $\bar{B}^{0}$ tagged events can be expressed as:

$$
f_{B_{\text {tag }}^{0} / \bar{B}_{\text {tag }}^{0}}=\frac{\Gamma}{4} \frac{1}{1-C \mu_{i} \zeta} e^{-\Gamma|\Delta t|}\left[\left(1 \pm \frac{\Delta D_{i}}{2}\right) \pm \mu_{i} D_{i}+\left(\mu_{i}\left(1 \pm \frac{\Delta D_{i}}{2}\right) \pm D_{i}\right) \cdot A(\Delta t)\right]
$$

where

$$
\begin{aligned}
& A(\Delta t)=-C \cos (\Delta m \Delta t)+S \sin (\Delta m \Delta t) \\
& \zeta=\frac{1}{1+\left(\tau_{B^{0}} \Delta m\right)^{2}} .
\end{aligned}
$$

The tagging asymmetry $\mu_{i}$, for a tagging category $i$, is defined as

$$
\mu_{i}=\frac{t_{i}-\bar{t}_{i}}{t_{i}+\bar{t}_{i}}
$$

where $t_{i}\left(\bar{t}_{i}\right)$ corresponds to the absolute tagging efficiency for $B^{0}\left(\bar{B}^{0}\right)$ in category $i$. For each tagging category $i$, the dilution coefficient due to mistagging $D_{i}$ and the dilution asymmetry $\Delta D_{i}$ are defined in terms of the probability of mistagging $B^{0}$ as $\bar{B}^{0}\left(\omega_{i}^{B^{0}}\right)$ and $\bar{B}^{0}$ as $B^{0}\left(\omega_{i}^{\bar{B}^{0}}\right)$ :

$$
\begin{gathered}
D_{i}=1-\omega_{i}^{B^{0}}-\omega_{i}^{\bar{B}^{0}} \simeq 1-2\left\langle\omega_{i}\right\rangle \\
\Delta D_{i}=-2\left(\omega_{i}^{B^{0}}-\omega_{i}^{\bar{B}^{0}}\right)=-2 \Delta \omega_{i} .
\end{gathered}
$$


In the following sections a more detailed overview of all important aspects of measuring time-dependent asymmetries is presented.

\subsection{1 $B$ meson flavor tagging}

Flavor tagging is a critical part of any time-dependent $C P$ violation measurement. Many BABAR physics analyses depend on the results of dedicated studies, in which special algorithms for $B$ meson flavor tagging were developed and tuned. In $B^{0} \rightarrow$ $K_{S}^{0} \pi^{0}$ and $B^{0} \rightarrow K_{S}^{0} \pi^{0} \gamma$ analyses two slightly different algorithms were used, where one represents an improved and redesigned version of the other.

Many different physics processes can be used to identify the flavor of the $B$ meson. One can use high momentum leptons corresponding to semi-leptonic $B$ decays, kaons and $\Lambda$ baryons coming from the cascade decays, slow pions from $D^{*}$ decays. Figure 4.1 shows diagrams corresponding to the semi-leptonic and cascade $B$ decays. The leptons in the two decays, corresponding to the same $B$ flavor, are different in sign, but the lepton from the direct semi-leptonic decay is more energetic, which makes it is possible to tag the $B$ meson flavor. In the case of the cascade decay, the $B$ meson flavor can be effectively correlated with the total charge of kaons.

The flavor tagging algorithm is implemented as a set of neural networks called subtaggers, where each neural net corresponds to one physics process. The output of the subtaggers is used as input to another neural net that determines the probability of the proper tagging for the entire event. Then the events are grouped in tagging categories 

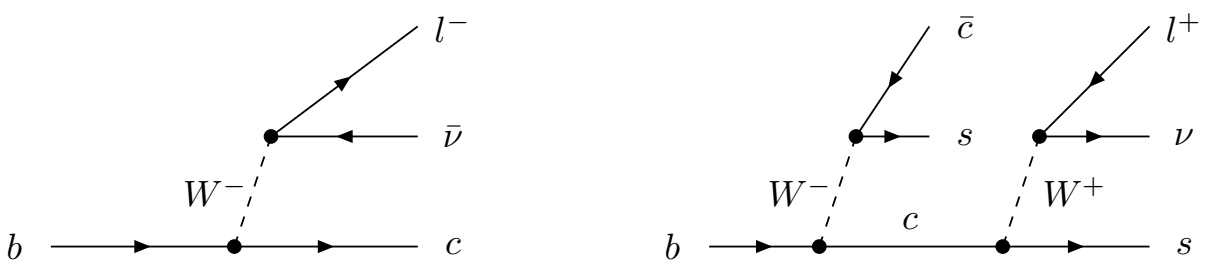

Figure 4.1: Feynman diagrams of a semi-leptonic (left) and a cascade (right) $\bar{B}$ meson decays.

with similar mis-tag fractions. The group with the best tagging performance is split in two categories, which separate the semi-leptonic decays from the rest of the processes. This is done to facilitate the study of the systematic effects of underlying physics.

For flavor tagging the tracks that are used for the $B_{C P}$ meson reconstruction are removed from the list of charged and neutral tracks that are used for flavor tagging of the $\operatorname{tag} B$ meson. The missing momentum in an event is determined from the 4-momenta of the beam and the $B_{C P}$ meson candidate.

All tagging algorithms rely on particle identification and it has direct impact on the overall performance of the flavor tagging. For all charged tracks in an event a particle hypothesis is assigned. If two or more hypotheses can be assigned to one track, the ambiguity is resolved in different ways by different algorithms. Some of the algorithms assign the hypothesis based on the likelihoods and some rely on special selection rules. The final list of tracks, which is essentially a list of all charged tracks with energy corrected according to the particle hypothesis, is used in the tagging algorithms to calculate the $B$ meson flavor.

There are three lepton sub-taggers, which use the following information as input: lepton candidates momenta in the center of mass frame, the angle between the can- 
didates and the direction of the missing momentum in the center of mass frame and charge of the candidates. They are trained to tag the $B$ meson flavor using electrons, muons and tracks that were not identified as leptons. The latter corresponds to a case when a real lepton from the direct semi-leptonic decay cannot be identified as a lepton, but it still can be useful for flavor tagging.

The kaon sub-tagger uses a list of the best three kaon candidates with low misidentification probability as input for the neural net. The kaons from the cascade decay $b \rightarrow c \rightarrow s$ have the same charge, which helps to flavor tag the $B$ meson. This tagger also uses the sum of the squares of the transverse momenta of charged tracks in the event as input.

The slow pion sub-tagger uses slow pions from the $D^{* \pm}$ decays to discriminate $B$ meson flavor. In order to suppress background, the slow pion is correlated with other tracks directions. Since the $D$ meson and the slow pion are created almost at rest in the $D^{*}$ rest frame, the slow pion is collinear to the $D$ meson direction in the $B$ meson rest frame, which is approximated as the $B_{\text {tag }}$ decay thrust axis, calculated in the $B_{\text {tag }}$ rest frame.

The other sub-taggers rely on the identification of such processes as the $\Lambda$ decays, which provide information about the $s$-quark flavor similar to the kaons. The fast-slow correlation tagger exploits the angular correlations between slow charged pions from $D^{*}$ decays and fast, oppositely charged tracks originating from the $W^{ \pm}$hadronization in the $b \rightarrow c W$ process. The kaon-slow pion tagger identifies $D *$ decays through the 
angular correlation of a charged kaon and a slow pion.

The redundancy of information in the sub-taggers is such, that if a sub-tagger is removed from the algorithm, the effective tagging performance degradation is just a few percent for all sub-taggers but the kaon sub-tagger.

A few different effects can lead to non-negligible tagging asymmetry. The major contribution to the asymmetry can be attributed to the neural net training, where an asymmetry in the training sample might lead to an asymmetry in the neural net output. Some physics processes can also lead to the tagging asymmetry. The reconstruction efficiency of charged kaons is asymmetric due to different cross-section of nuclear interaction of $K^{+}$and $K^{-}$. This effect is of the order of few percents. The particle identification fake rates can also have charge asymmetry. To account for such effects one has to allow for non-zero flavor tagging asymmetry in the time-dependent $C P$ violation measurements.

One of the most useful ways to present the flavor tagging performance is to calculate the effective tagging efficiency, which is defined as:

$$
Q=\epsilon D^{2}
$$

where $\epsilon$ is the absolute tagging efficiency and $D$ is the dilution coefficient. In general the uncertainty on a measured asymmetry depends on the effective tagging efficiency 


\begin{tabular}{|l|cc|cc|cc|}
\hline Category & $\epsilon(\%)$ & $\Delta \epsilon(\%)$ & $\omega(\%)$ & $\Delta \omega(\%)$ & $Q(\%)$ & $\Delta Q(\%)$ \\
\hline \hline Leptons & $9.8 \pm 0.1$ & $-0.3 \pm 0.2$ & $3.9 \pm 0.7$ & $3.3 \pm 1.5$ & $8.4 \pm 0.3$ & $-1.5 \pm 0.6$ \\
Kaon I & $16.8 \pm 0.2$ & $0.5 \pm 0.3$ & $8.9 \pm 0.6$ & $0.9 \pm 1.2$ & $11.4 \pm 0.3$ & $-0.2 \pm 0.7$ \\
Kaon II & $19.4 \pm 0.2$ & $-0.5 \pm 0.3$ & $21.0 \pm 0.6$ & $5.0 \pm 1.2$ & $6.6 \pm 0.3$ & $-2.4 \pm 0.6$ \\
Others & $20.2 \pm 0.2$ & $-0.2 \pm 0.4$ & $31.0 \pm 0.6$ & $6.2 \pm 1.2$ & $3.0 \pm 0.2$ & $-1.9 \pm 0.4$ \\
\hline \hline Total & $66.3 \pm 0.3$ & $-0.6 \pm 0.6$ & & & $29.3 \pm 0.6$ & $-6.0 \pm 1.1$ \\
\hline
\end{tabular}

Table 4.1: Performance of Moriond tagger on data Run1-4.

in the following way:

$$
\sigma \propto \frac{1}{\sqrt{Q}}
$$

The performance of a tagging algorithm is measured in data on a special sample of exclusively reconstructed $B$ decays called the BRECO sample. This sample consists of events corresponding to charged and neutral $B$ decays, for which at least one of the $B$ decays is fully reconstructed. Even though some tagging categories have different performance for charged and neutral $B$ decays, the charged sample is still useful for the algorithm validation.

The performance of the two taggers used in the analyses is shown in Table 4.1 (Moriond tagger) and Table 4.2 (Tag04 tagger).

\subsubsection{Reconstruction of the $B$ meson decay vertices for par- tially reconstructed decays}

In order to determine the decay time difference between two $B$ mesons with only the $B_{C P}$ meson decay fully reconstructed, a special vertexing algorithm is employed that allows to determine the other $B_{t a g}$ meson decay vertex position using tracks that are 


\begin{tabular}{|l|cc|cc|cc|}
\hline Category & $\epsilon(\%)$ & $\Delta \epsilon(\%)$ & $\omega(\%)$ & $\Delta \omega(\%)$ & $Q(\%)$ & $\Delta Q(\%)$ \\
\hline \hline Leptons & $9.0 \pm 0.1$ & $-0.0 \pm 0.2$ & $3.5 \pm 0.8$ & $1.7 \pm 1.5$ & $7.8 \pm 0.3$ & $-0.6 \pm 0.5$ \\
Kaon 1 & $11.0 \pm 0.1$ & $0.3 \pm 0.3$ & $4.6 \pm 0.7$ & $1.1 \pm 1.4$ & $9.1 \pm 0.3$ & $-0.2 \pm 0.6$ \\
Kaon 2 & $17.1 \pm 0.2$ & $-0.3 \pm 0.3$ & $15.9 \pm 0.6$ & $1.0 \pm 1.2$ & $8.0 \pm 0.3$ & $-0.6 \pm 0.6$ \\
Kaons-Pions & $13.8 \pm 0.1$ & $0.0 \pm 0.3$ & $24.6 \pm 0.7$ & $0.1 \pm 1.4$ & $3.5 \pm 0.2$ & $-0.0 \pm 0.4$ \\
Pions & $14.5 \pm 0.1$ & $0.4 \pm 0.3$ & $33.3 \pm 0.7$ & $-9.1 \pm 1.5$ & $1.8 \pm 0.1$ & $1.8 \pm 0.3$ \\
Others & $9.8 \pm 0.1$ & $-0.2 \pm 0.2$ & $41.0 \pm 0.9$ & $-4.1 \pm 1.8$ & $0.3 \pm 0.1$ & $0.3 \pm 0.1$ \\
\hline \hline Total & $75.2 \pm 0.3$ & $0.2 \pm 0.7$ & & & $30.5 \pm 0.6$ & $0.7 \pm 1.1$ \\
\hline
\end{tabular}

Table 4.2: Performance of Tag04 tagger on data Run1-4.

not associated with the fully reconstructed $B$ meson. Since only small fraction of the generic $B$ meson decays can be exclusively reconstructed, the algorithm relies on inclusive techniques with a partial $B_{\text {tag }}$ reconstruction to keep the overall efficiency of the time-dependent measurements high.

The $B_{t a g}$ vertex reconstruction is complicated by the presence of secondary vertices, corresponding to decays of long live particles. First of all, the algorithm identifies the decay vertices of the $K_{S}$ 's and $\Lambda$ 's and replaces tracks corresponding to the decay products with just one composite particle in the list of tracks that do not belong to the $B_{C P}$ candidate. If the decay vertex of the $K_{S}$ or $\Lambda$ is found outside of the SVT or it does not have enough information from the SVT to accurately measure its position and the corresponding composite particle direction, then it is removed from the list of tracks that are used for the final $B_{\text {tag }}$ vertex fit.

In general the $B_{t a g}$ vertex resolution is much worse than the resolution on the exclusively reconstructed $B$ meson vertex. The reason for this is the multiple Coulomb scattering in the beam-pipe and SVT, which significantly degrades the directional resolution for low momentum charged tracks. The average multiplicity of long lived 
charged tracks is quite high and therefore they typically have low momentum. The amount of material in the beam-pipe corresponds to about $1 \%$ of the radiation length and on average the scatter angle is expected to be:

$$
\delta \theta \approx \frac{13.6 \mathrm{MeV}}{\beta p} \sqrt{x / X_{0}}
$$

where $\beta$ is the particle velocity, $p$ its momentum and $x / X_{0}$ is the thickness of the scattering medium in radiation lengths. Typically charged tracks from $B$ meson decays have low $\beta p$, which is on average of the order of $0.5 \mathrm{MeV}$ or less. Taking into account the radius of the beam-pipe of $2.5 \mathrm{~cm}$, one would expect to have smearing of the $z$-position resolution due to multiple scattering on the beam-pipe of the order of $70 \mu \mathrm{m}$ for a charge track of $500 \mathrm{MeV}$ momentum moving perpendicular to the beam direction. If the particle has a significant longitudinal momentum, the resolution becomes even worse and the uncertainty in the $z$-projection of the vertex increases. This happens because the flight distance to the beam-pipe becomes bigger and the amount of material transversed by the particle increases.

A significant number of the secondary vertices are due to $D$ and $D_{S}$ meson decays. Their average lifetime is smaller, but comparable with the lifetime of the $B_{d}$ mesons, which makes it difficult to separate the $D$ and $D_{S}$ decay vertices from the $B$ meson vertices. In practice just a small fraction of the $D$ and $D_{S}$ vertices can be reconstructed and replaced with a composite track, therefore the impact on the overall $B_{t a g}$ vertexing performance is negligible. The existence of the secondary vertices 

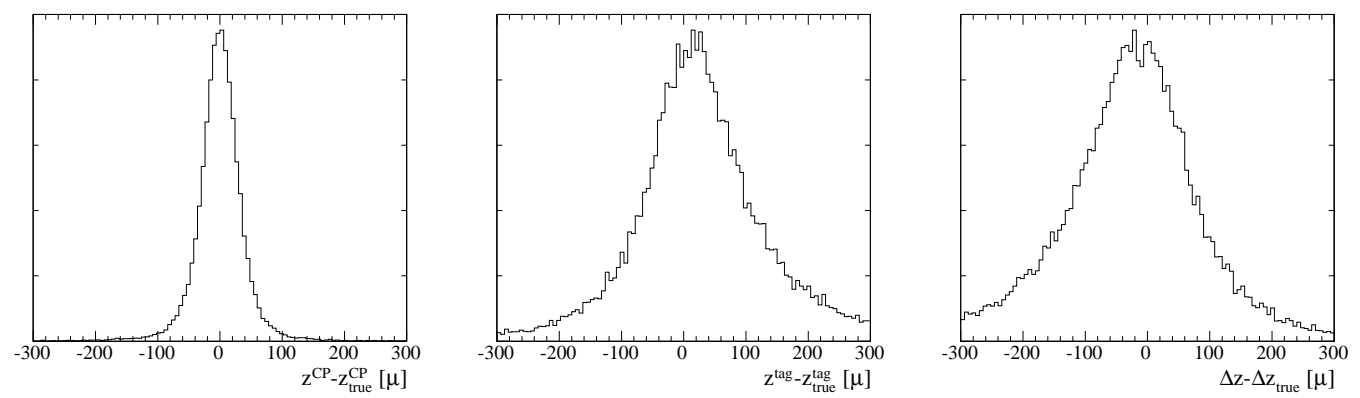

Figure 4.2: Resolution distributions for the $B_{C P}$ and $B_{\text {tag }}$ decay vertices and the distance between them along the $z$-axis for a sample of Monte Carlo generated $B^{0} \rightarrow$ $\pi^{+} \pi^{-}$decays.

of this type limits the accuracy of the time-dependent measurements, since tracks corresponding to $D$ and $D_{S}$ can bias the $B_{t a g}$ vertex position measurements. This effect is called the charm bias.

In order to minimize the effect of the charm bias the vertexing algorithm was modified such that tracks with large contribution to the vertex fit $\chi^{2}$ can be removed from the fit. If the fit to the $B_{t a g}$ vertex is poor, then the algorithm tries to improve the fit by removing tracks with high $\chi^{2}$ and refit the vertex again.

Figure 4.2 shows the vertex resolution of $B_{C P}$ and $B_{\text {tag }}$ mesons along the $z$-axis and the resolution on $\Delta Z$ using a sample of Monte Carlo simulated $B \rightarrow \pi^{+} \pi^{-}$events, which have exceptional $B_{C P}$ decay vertex resolution. The average error in the $B_{C P}$ vertex position is about $30 \mu \mathrm{m}$ and in the $B_{\text {tag }}$ it is about $95 \mu \mathrm{m}$, which results in the average error in $\Delta Z$ of the order of $105 \mu \mathrm{m}$. 


\subsection{3 $\Delta z$ to $\Delta t$ transformation}

When the vertices of the two $B$ mesons in the event are found it is possible to calculate the decay time difference $(\Delta t)$. By definition the $\Delta t$ is:

$$
\Delta t=t_{\mathrm{CP}}-t_{\mathrm{tag}}
$$

where $t_{C P}$ and $t_{\text {tag }}$ are the proper decay time of the $B_{\mathrm{CP}}$ and $B_{\text {tag }}$ mesons respectively. The $z$-position of the vertices in the LAB frame, $z_{C P}$ and $z_{\text {tag }}$, define the $\Delta z$ :

$$
\Delta z=z_{\mathrm{CP}}-z_{\mathrm{tag}}
$$

The vertex position and the decay time are defined in different frames, which makes the relation between the $\Delta z$ and $\Delta t$ non trivial. Fortunately the relative boost of the $B$ meson rest frame, with respect to the center of mass frame (the rest frame of the $\Upsilon(4 S))$, is quite small $\left(\beta^{*} \approx 0.06, \gamma^{*} \approx 1.002\right)$, which simplifies the calculation of the $\Delta t$. Neglecting the small momentum of the $B$ meson in the center of mass frame one would get:

$$
\Delta t \approx \frac{\Delta z}{\gamma \beta_{z} c}
$$

where $\gamma \beta_{z} \approx 0.56$, which corresponds to the boost of the center of mass frame with respect to the laboratory frame. If we take into account the relative boost of the $B$ 
meson rest frame with respect to the center of mass frame, then:

$$
\Delta z=z_{C P}-z_{t a g}=\left(\gamma \beta_{z}\right)_{C P} t_{C P}-\left(\gamma \beta_{z}\right)_{t a g} t_{t a g}
$$

where

$$
\begin{aligned}
\left(\gamma \beta_{z}\right)_{B}= & \frac{p_{L A B}}{m_{B}}=\frac{1}{m_{B}} \gamma\left(p_{C M S}+\beta_{z} E_{C M S}\right)= \\
& \frac{1}{m_{B}}\left(\gamma \gamma^{*} \beta^{*} m_{B}+\gamma \gamma^{*} \beta_{z} m_{B}\right)= \\
& \gamma \gamma^{*} \beta_{z}\left(1+\frac{\beta^{*}}{\beta_{z}} \cos \theta_{B}\right)
\end{aligned}
$$

neglecting $\gamma^{*} \approx 1.002$ we get

$$
\Delta z=\gamma \beta_{z} \Delta t+\gamma \beta^{*} \cos \theta_{B}\left(t_{C P}+t_{t a g}\right)
$$

where $\theta_{B}$ is the angle between the $B$ meson direction and the $z$-axis in the center of mass frame.

We don't measure the sum of the lifetimes, but since its contribution is suppressed by a factor of $\frac{\beta^{*}}{\beta_{z}} \cos \theta_{B} \approx 0.13 \cos \theta_{B}$ we expect that this effect is small. On average the second term is zero. Taking into account the angular distribution of the $B$ meson in the $\Upsilon(4 S)$ frame, which corresponds to a two-body decay of a vector meson with non-zero spin projection along its momentum to two pseudo-scalars, $d \sigma \propto \sin ^{2} \theta_{B} d \Omega$, the RMS of the second term is about $2 \beta^{*}(c \tau)_{B} / \sqrt{5} \approx 26 \mu m$. With a typical resolution 
on $\Delta z$ of $100 \mu m$ this effect amounts to about $3 \%$ degradation in the $\Delta t$ resolution, hence it can be neglected in the time-dependent asymmetry measurements.

\subsubsection{Resolution model}

The resolution on the measurements of $\Delta t$ is parameterized as a sum of three Gaussian distributions. The mean and the variance of the core and tail Gaussian distributions depend on the error on $\Delta t$ and they are calculated on an event by event basis, which allows to weigh properly the contributions of events with small and large errors on $\Delta t$. The last distribution has a fixed width and is primarily used to account for the outliers. The resolution function $\mathcal{R}\left(\Delta t, \sigma_{\Delta t}\right)$ is expressed as,

$$
\begin{aligned}
\mathcal{R}\left(\Delta t, \sigma_{\Delta t}\right)= & \frac{f_{\text {core }}}{S_{\text {core }} \sigma_{\Delta t} \sqrt{2 \pi}} \exp \left[-\frac{1}{2}\left(\frac{\delta t-b_{\text {core }} \sigma_{\Delta t}}{S_{\text {core }} \sigma_{\Delta t}}\right)^{2}\right]+ \\
& \frac{f_{\text {tail }}}{S_{\text {tail }} \sigma_{\Delta t} \sqrt{2 \pi}} \exp \left[-\frac{1}{2}\left(\frac{\delta t-b_{\text {tail }} \sigma_{\Delta t}}{S_{\text {tail }} \sigma_{\Delta t}}\right)^{2}\right]+ \\
& \frac{f_{\text {outl }}}{\sigma_{\text {outl }} \sqrt{2 \pi}} \exp \left[-\frac{1}{2}\left(\frac{\delta t}{\sigma_{\text {outl }}}\right)^{2}\right]
\end{aligned}
$$

where $\delta t=\Delta t_{\text {meas }}-\Delta t_{\text {true }}$ is the residual of $\Delta t$. The scale factors $S_{\text {core }}$ and $S_{\text {tail }}$ of the variance $\left(\sigma_{\Delta t}\right)$ allow to correct for an overestimation or underestimation of the error on $\Delta t$. The Gaussian mean parametrized as $b_{\text {core }} \Delta t$ or $b_{\text {tail }} \Delta t$ allows to correct for the charm bias. The dependence of the bias on $\Delta t$ from $\sigma_{\Delta t}$ results on the dependence of $\sigma_{\Delta t}$ from the angle between the $z$-axis and the charm meson momentum in the laboratory reference frame. When the angle is small the decay products of the charm 

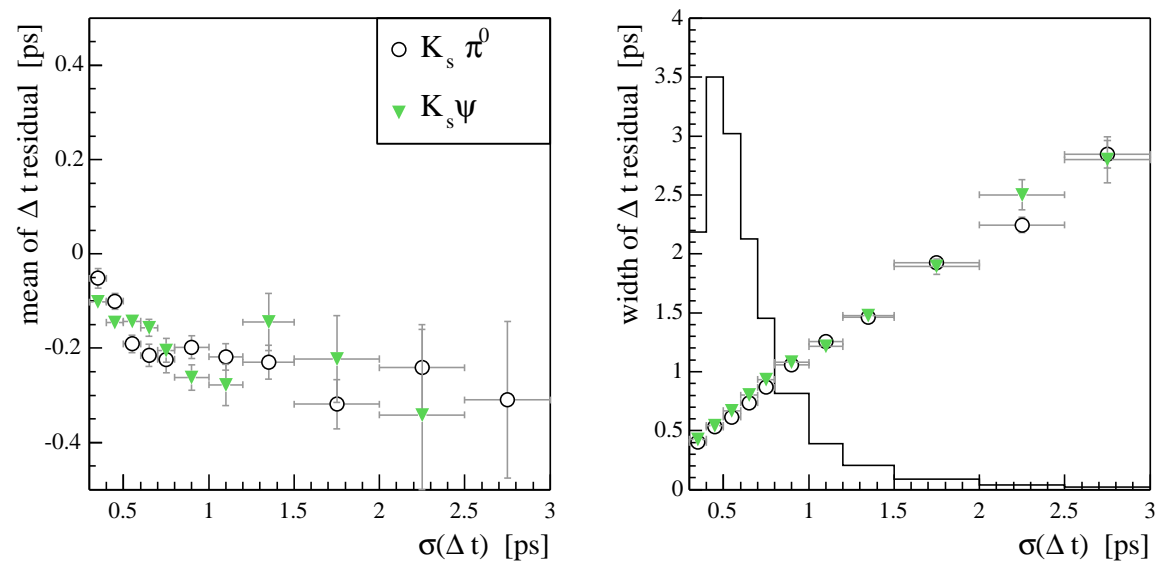

Figure 4.3: Mean and width of the $\Delta t$ bias, $\Delta t-\Delta t_{\text {true }}$, versus $\sigma_{\Delta t}$ for $B^{0} \rightarrow K_{S}^{0} \pi^{0}$ and nominally reconstructed $B^{0} \rightarrow J / \psi K_{S}$ candidates. The histogram on the right plot displays the distribution of $\sigma_{\Delta t}$.

meson have poor vertex resolution in the $z$-direction and at the same time the charm bias of the $B$ meson vertex position becomes larger. When the charm meson flies perpendicular to the $z$-axis, the charm bias vanishes and the charged tracks from the charm decay have better $z$ vertex resolution. Figure 4.3 shows the RMS of the residual of $\Delta t$ versus the estimated error on $\Delta t$ and the mean of the residual versus the error on $\Delta t$ for a signal Monte Carlo sample.

\subsubsection{The beam constrained vertex reconstruction}

The reconstruction of the $B$ meson decay vertex position of $B^{0} \rightarrow K_{S}^{0} \pi^{0}$ and $B^{0} \rightarrow$ $K_{S}^{0} \pi^{0} \gamma$ decays is complicated by the absence of charged tracks among the final state particles that directly originate from the $B$ meson vertex. In order to overcome this issue other constraints such as small transverse movement of the $B$ meson, small size 
and known position of the luminous region and large boost along the beam direction can be exploited.

If the $K_{S}$ is reconstructed in decays to two charged pions, neglecting the $B$ meson transverse motion, we can estimate the $B$ decay vertex position by intersecting the $K_{S}$ flight direction with the beam transverse profile. The intrinsic beam size is about $4 \mu m$ in $y, 200 \mu m$ in $x$ and about $8 \mathrm{~mm}$ in $z$. The beam spot position and its size are determined for each run with much better precision in $x$ and about $5 \mu m$ in $y$. In order to correct for the neglected transverse $B$ meson motion, one can either inflate errors on the beam spot size or try to fit the whole $\Upsilon(4 S)$ decay tree constraining the sum of $B$ meson decay times. In the latter case, the tag $B$ meson is replaced with the inclusively reconstructed decay vertex and its 4-momentum is determined from the conservation of energy and momentum.

The uncertainty in the $K_{S}$ meson direction dominates the uncertainty on the $z$ position of the $B$ meson decay vertex. Two major factors contribute to the directional resolution of the $K_{S}$ trajectory. First of all the resolution depends on where the $K_{S}$ meson decays in the detector. If it decays outside the 4th layer of the SVT, then the resolution of the $K_{S}$ meson direction is inadequate to reconstruct the $B$ meson decay vertex with precision required for the time-dependent asymmetry measurements. Another factor that defines the ultimate vertex resolution is the angle between $K_{S}$ direction and the $z$-axis. For small angles the $z$-projection of the $B$ meson decay vertex becomes very large. Figure 4.4 illustrates the effect of the $K_{S}$ meson decay 

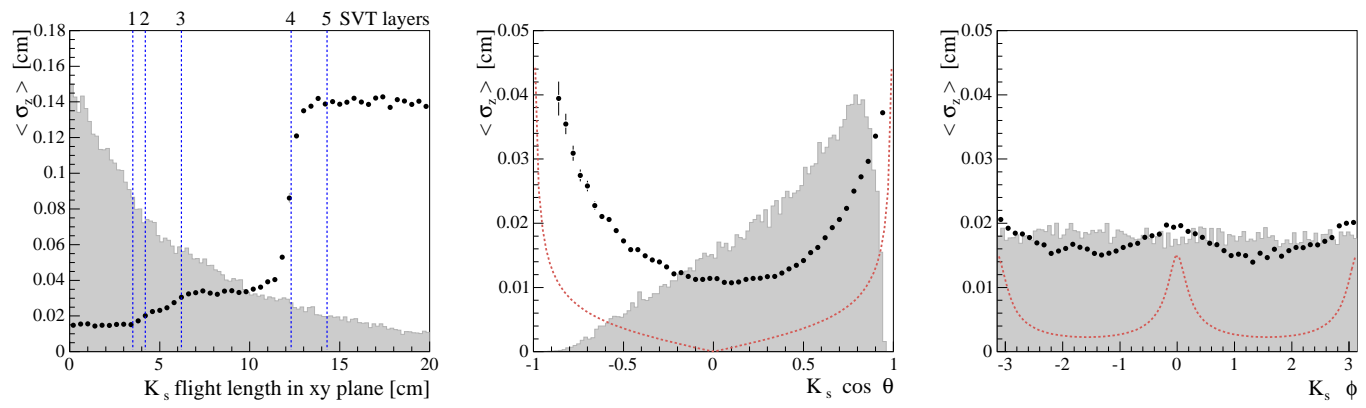

Figure 4.4: The effect of the $K_{S}$ decay vertex position and flight direction on the $B$ meson decay vertex resolution. The left plot shows an average estimated uncertainty in $z_{B_{C P}}$ (dots) as a function of the $K_{S}$ decay length. The dashed lines indicate the position of the five SVT layers. The superimposed histogram shows the decay length distribution with an arbitrary scale. The other two plots show the estimated uncertainty as a function of the $K_{S}$ polar (middle) and azimuthal (right) angles. The dashed lines denote the contribution of the uncertainty in the beam-spot.

length and its polar and azimuthal angles on the $B$ meson decay vertex resolution.

It is convenient to separate all events in 4 classes:

- Class I (red) - decays where both pions from $K_{S} \rightarrow \pi^{+} \pi^{-}$decays have at least

$1 \phi$ and $1 z$ hit in any of the first three inner layers of SVT.

- Class II (blue) - decays where both pions have at least $1 \phi$ and $1 z$ hit in the SVT, but not in class I. These events mostly corresponds to $K_{S}$ decays beyond the inner 3 SVT layers.

- Class III (black) - decays where either of the two pions have at least one SVT hit, but do not satisfy the requirements of class I or II.

- Class IV (green) - decays where neither pion has any SVT hits.

Figure 4.5 shows distributions of the estimated uncertainty in $\Delta t, \sigma_{\Delta t}$, for these 

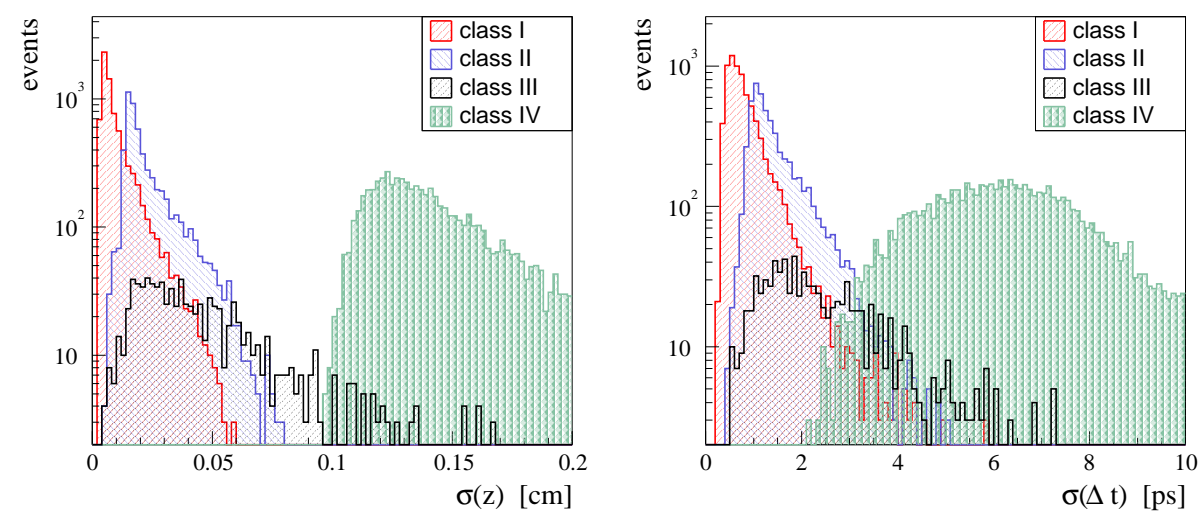

Figure 4.5: Distribution for the estimated uncertainty in $z_{B^{0}}$ (left) and $\Delta t$ )right) for different classes of $B^{0} \rightarrow K_{S}^{0} \pi^{0}$ decays.

classes.

Normally events with an uncertainty on $\Delta t$ above 2.5 ps are not used for measurements of the $C P$ violating asymmetries. This essentially excludes all events of class IV and large fraction of events of class III from such measurements. The relative fraction of events of class I and II depends on the typical momentum of the $K_{S}$. In the case of the $B^{0} \rightarrow K_{S}^{0} \pi^{0} \gamma$ decays the $K_{S}$ is less energetic than in the case of $B^{0} \rightarrow K_{S}^{0} \pi^{0}$ decays, which increases the fraction of usable events for the extraction of the $S$ asymmetry term.

In order to validate this type of $B$ meson decay vertex reconstruction, extensive studies were performed using Monte Carlo simulations and data. A sample of $B^{0} \rightarrow J / \psi K_{S}$ decays was reconstructed using two different vertexing techniques: the nominal one and a "mangled" one, where the information from the $J / \psi$ was not used for the $B$ meson decay vertex reconstruction and the vertex was reconstructed only 
using the $K_{S}$ meson. As a cross check, a sample of $B^{+} \rightarrow K_{S} \pi^{+}$events was used as well, with the charged pion originating directly from the $B$ meson not used for the decay vertex reconstruction. Figure 4.6 provides a comparison of the vertex reconstruction performance for different vertexing techniques for Monte Carlo simulated events.

A comparison of the $B$ meson decay vertex reconstruction for $B^{0} \rightarrow J / \psi K_{S}$ events in data using the nominal and mangled vertexing indicates that no bias was introduced by the beam spot constraint. From the difference between the $\Delta t$ reconstructed with and without the $J / \psi$ vertex we define a pull in $\Delta t$ as,

$$
\chi(\Delta t)=\frac{\Delta t_{w / o}-\Delta t_{w /}}{\sqrt{\sigma(\Delta t)_{w / o}^{2}-\sigma(\Delta t)_{w /}^{2}}}
$$

where it is assumed that the two estimates are fully correlated. Figure 4.7 shows the $\chi(\Delta t)$ distribution for data and Monte Carlo for class I and II events. The pulls are roughly $10 \%$ wider in data than in Monte Carlo. This small discrepancy is used to scale the resolution function as an estimate of the systematic uncertainty arising from the vertexing technique.

\subsection{Continuum background suppression}

It was mentioned earlier, that the continuum background events corresponding to $e^{+} e^{-} \rightarrow q \bar{q}$ decays, where $q$ is either up, down, strange or charm quark, represent the 

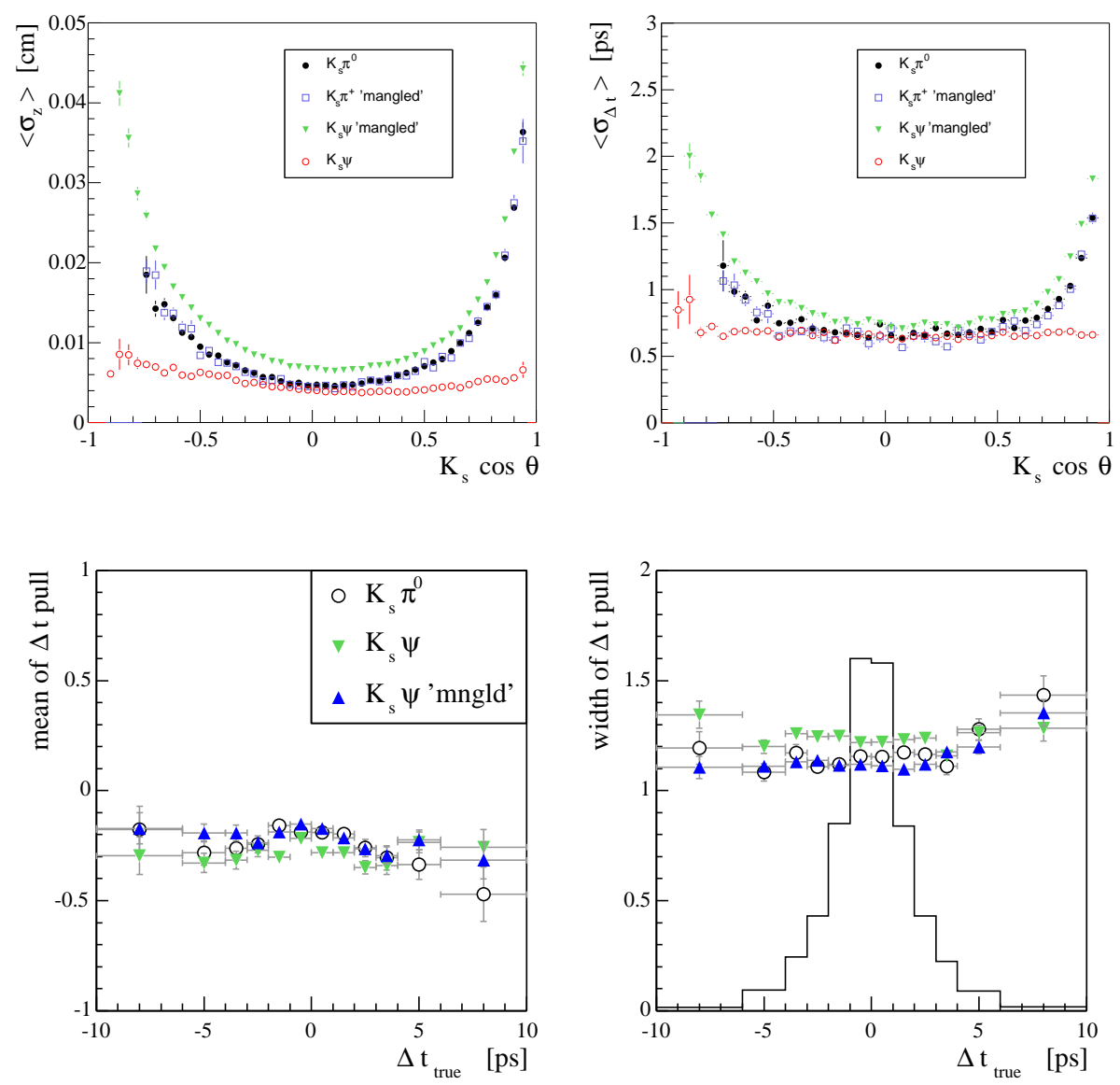

Figure 4.6: The top plots show the average estimated error in the $z$ position of the $B$ meson vertex (left) and in $\Delta t$ (right) as a function of the cosine of the $K_{S}$ polar angle for the different Monte Carlo samples for class I events. The bottom plots show the width and mean of the $\Delta t$ pull, $\delta(\Delta t) \equiv\left(\Delta t-\Delta t_{\text {true }}\right) / \sigma_{\Delta t}$, versus $\Delta t_{\text {true }}$. The histogram displays the distribution of $\Delta t_{\text {true }}$. The bias in the mean of $\Delta t$ pull (left plot) shows the effect of the charm bias, which is taken into account in the resolution model. 

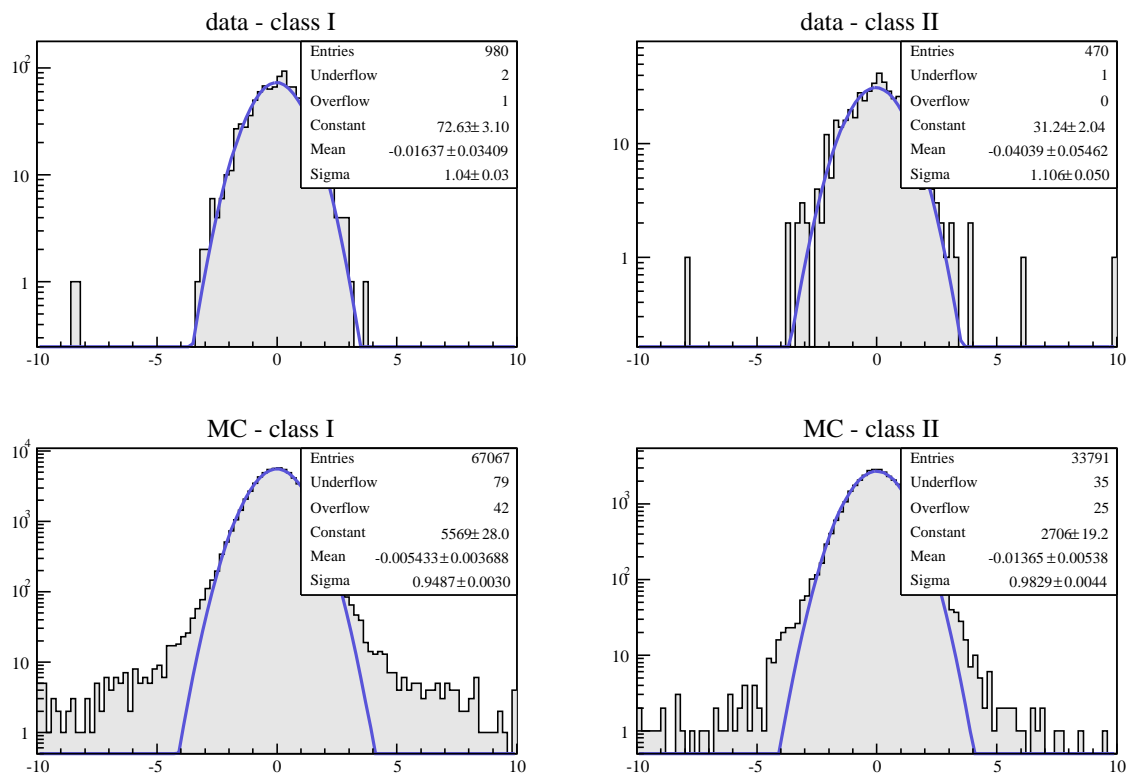

Figure 4.7: Mangled-minus-nominal pulls for $\Delta t$ in $B^{0} \rightarrow J / \psi K_{S}$ in data (top) and Monte Carlo (bottom) for class I (left) and class II (right) events.

dominant background in analyses involving rare $B$ meson decays.

There are two effective ways to separate the continuum background from $B \bar{B}$ events. The first exploits the idea of the event shape difference. The continuum events are jet like in the center of mass frame and the $B \bar{B}$ events are spherically symmetric in that frame. Another way to suppress the continuum background comes from the difference in kinematics of the physics processes. Detection of an energetic lepton coming from the semi-leptonic $b$ or $c$ quark decays allows to essentially remove most of the continuum $u \bar{u}, d \bar{d}$ and $s \bar{s}$ background events. Only about $9 \%$ of the $B$ meson decays can be effectively tagged as the semi-leptonic decays and therefore it is advantageous to combine these events into a separate category. These events have much better signal to background ratio than other events and classifying events 
by their signal to background ratio allows to optimize statistical precision of measurements. In practice all events are grouped by the flavor tagging performance to minimize the complexity of the final fit.

There are many different ways to parameterize the event shape information. Current analyses use a simple parameterization, which doesn't require dedicated optimization and achieves the background suppression performance comparable with other methods. Utilization of the neural network might slightly improve the continuum background suppression, but the effect is essentially negligible. It is possible to define an angular moment $L_{i}$ as,

$$
L_{i}=\sum_{k}\left|p_{k}\right|\left|\cos \theta_{k}\right|^{i}
$$

where $p_{k}$ is the momentum of the particle $k$ in the center of mass frame and the sum runs over all tracks besides the exclusively reconstructed $B$ meson daughters. $\theta_{k}$ is the angle between the particle direction and the thrust axis $\hat{n}$ of the $B_{C P}$ candidate in the center of mass frame. The thrust axis is defined as an axis that maximizes the thrust $T$ defined as:

$$
T=\frac{\sum_{k}\left|\hat{n} \cdot \overrightarrow{p_{k}}\right|}{\sum_{k}\left|p_{k}\right|} .
$$

In the case of the $B^{0} \rightarrow K_{S}^{0} \pi^{0}$ decays, the thrust axis is essentially parallel to the $K_{S}$ and $\pi^{0}$ flight direction in the center of mass frame, because the two body decay of the $B$ meson looks essentially the same way in the center of mass frame and in 


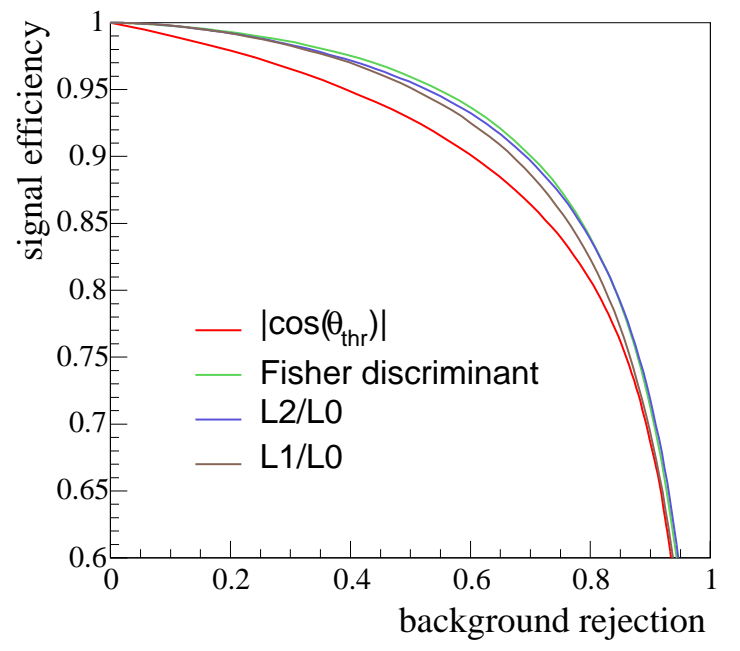

Figure 4.8: Efficiency versus continuum background rejection for several event shape variables for $B^{0} \rightarrow K_{S}^{0} \pi^{0}$ decays.

the $B$ meson rest frame due to small momentum of the $B$ meson with respect to the momentum of its decay products. In the case of the $B^{0} \rightarrow K_{S}^{0} \pi^{0} \gamma$ the thrust axis is to a good approximation parallel to the photon direction, since at the quark level this decay corresponds to $b \rightarrow s \gamma$ two body process.

The background discriminating variable can be defined as a ratio of two angular moments:

$$
R \equiv \frac{L_{2}}{L_{0}}=\frac{\sum_{k}\left|p_{k}\right| \cos ^{2} \theta_{k}}{\sum_{k}\left|p_{k}\right|} .
$$

Figure 4.8 shows the signal efficiency versus the background rejection for 4 different event shape discriminators, which demonstrate that the separation power of all the discriminators is quite similar.

It was found that the event shape is correlated with the tagging category. This 
correlation is taken into account by parameterizing the signal and background PDFs in different categories separately.

\subsection{Kinematic variables for the $B$ meson selection}

The electron-positron collisions allow to use the known beam energy and the total 4-momentum of the $e^{+} e^{-}$pair to over-constraint the $B \bar{B}$ meson pair in $e^{+} e^{-} \rightarrow$ $\Upsilon(4 s) \rightarrow B \bar{B}$ decays. Essentially we have two kinematic constraints that can be used to define and reconstruct exclusive $B$ decays. One of them is the mass of the exclusively reconstructed $B_{C P}$ meson, which is calculated from its 4-momentum and the other is the mass of the second $B_{t a g}$ meson, whose 4-momentum is determined from the known total 4-momentum of the $e^{+} e^{-}$pair minus the 4-momentum of the $B_{C P}$ meson. This is not a unique way to represent the two constraints and the selection of a proper basis depends on specific conditions of an analysis.

The most widely used set of kinematic variables, which are Lorentz invariant are defined in the following way:

$$
\begin{aligned}
m_{\mathrm{ES}} & =\sqrt{\frac{\left(s / 2+\vec{p}_{\Upsilon(4 S)} \cdot \vec{p}_{B_{C P}}\right)^{2}}{E_{\Upsilon(4 S)}^{2}}-\vec{p}_{B_{C P}}^{2}} \\
\Delta E & =\frac{E_{\Upsilon(4 S)} E_{B_{C P}}-\vec{p}_{\Upsilon(4 S)} \cdot \vec{p}_{B_{C P}}-s / 2}{\sqrt{s}}
\end{aligned}
$$

where $s$ is square of the center of mass energy, $\left(E_{\Upsilon(4 S)}, \vec{p}_{\Upsilon(4 S)}\right)$ and $\left(E_{C P}, \vec{p}_{C P}\right)$ are 4momentums of the $\Upsilon(4 S)$ resonance and the $B$ meson respectively. These equations 
become much simpler and more intuitive when calculated in the center of mass frame, where $\sqrt{s}=E_{\Upsilon(4 S)}^{*}$ and $\vec{p}_{\Upsilon(4 S)}=0$.

$$
\begin{aligned}
m_{\mathrm{ES}}{ }^{*} & =\sqrt{\left(\frac{1}{2} E_{\Upsilon(4 S)}^{*}\right)^{2}-{\overrightarrow{p^{*}}}_{B_{C P}}^{2}} \\
\Delta E^{*} & =E_{B_{C P}}^{*}-\frac{1}{2} E_{\Upsilon(4 S)}^{*}
\end{aligned}
$$

where $E_{B_{C P}}^{*}$ and $\vec{p}^{*} B_{C P}$ are energy and momentum of the $B$ meson in the center of mass frame.

This set of variables works fine for many exclusive $B$ meson decays. In most cases $\Delta E$ and $m_{\mathrm{ES}}$ have a very small correlation, which justifies the factorization of the probability distribution functions (PDFs) that enter the likelihood of the final fit, i.e.:

$$
P\left(m_{\mathrm{ES}}, \Delta E\right)=P\left(m_{\mathrm{ES}}\right) P(\Delta E)
$$

where $P\left(m_{\mathrm{ES}}, \Delta E\right)$ is a combined $\mathrm{PDF}$ of $m_{\mathrm{ES}}$ and $\Delta E, \mathrm{P}\left(m_{\mathrm{ES}}\right)$ is $m_{\mathrm{ES}} \mathrm{PDF}$ and $\mathrm{P}(\Delta E)$ is $\Delta E \mathrm{PDF}$. If the correlation is ignored and the factorization of the PDFs is used in the fit, the signal significance of the fit result will suffer and the result can be biased.

To understand why the correlation is small, it is important to understand what makes the largest contribution to the uncertainty in $m_{\mathrm{ES}}$. To get an answer to this question it is illustrative to express $m_{\mathrm{ES}}$ in the laboratory reference frame using the 
energy of the beams:

$$
m_{\mathrm{ES}}=\sqrt{\left(\frac{2 E_{H E R} E_{L E R}}{E_{H E R}+E_{L E R}}+\frac{E_{H E R}-E_{L E R}}{E_{H E R}+E_{L E R}}\left|p_{B_{C P}}\right| \cos \theta\right)^{2}-\vec{p}_{B_{C P}}^{2}}
$$

where $E_{H E R}$ is the energy of the high energy electron beam and $E_{L E R}$ is the energy of the low energy positron beam. For a typical set of parameters $\left(E_{H E R}=9 \mathrm{GeV}\right.$, $\left.E_{L E R}=3.1 \mathrm{GeV}, p_{B_{C P}} \approx 2.98 \mathrm{GeV}, \theta \approx 0.070\right)$, where $\theta$ is the polar angle of the $B$ meson with respect to the beam direction, the expected errors on $m_{\mathrm{ES}}$ can be expressed in the following way:

$$
\sigma_{m_{\mathrm{ES}}} \approx \sqrt{\left(0.85 \sigma_{E_{L E R}}\right)^{2}+\left(0.30 \sigma_{E_{H E R}}\right)^{2}+\left(117 \mathrm{MeV} \sigma_{\theta}\right)^{2}+\left(0.006 \sigma_{p_{B}}\right)^{2}}
$$

The uncertainty in $\Delta E$ is completely dominated by the energy resolution of the $B$ meson. Taking the energy spread of the HER beam 5.5 MeV and the LER beam 2.3 $\mathrm{MeV}$ as the errors on their energy and ignoring small terms one would get:

$$
\sigma_{m_{\mathrm{ES}}} \approx \sqrt{(2.6 \mathrm{MeV})^{2}+\left(117 \mathrm{MeV} \sigma_{\theta}\right)^{2}}
$$

A typical angular resolution of the $B$ meson candidate, $\sigma_{\theta}$, for final states consisting mostly from charged tracks, is quite good. For example, for $B^{0} \rightarrow \pi^{+} \pi^{-}$it is about 7 mrad and the expected error on $m_{\mathrm{ES}}$ is about $2.7 \mathrm{MeV}$, which makes the uncertainty in the beam energy the dominant contribution to the uncertainty in $m_{\mathrm{ES}}$ and the 
correlation with $\Delta E$ is small.

The picture changes dramatically if the final state of the $B$ meson has many neutral tracks that are reconstructed based only on the calorimeter information. For example for $B^{0} \rightarrow K^{* 0} \gamma$ the $\sigma_{\theta}$ on average is about $20 \mathrm{mrad}$. This make a substantial contribution to the uncertainty on $m_{\mathrm{ES}}$ and the expected error on $m_{\mathrm{ES}}$ is about 3.5 MeV. It is interesting to understand why the $\sigma_{\theta}$ is so large in this case. The average angular resolution of the $K^{*}$ and photon is $5 \mathrm{mrad}$ and $4 \mathrm{mrad}$ respectively, which is significantly smaller than $\sigma_{\theta} \approx 20 \mathrm{mrad}$ of the $B$ meson itself. The reason for this is the uncertainty in momenta of the $K^{*}$ and photon, which enter the calculation of the $B$ meson direction.

$$
\theta \approx \frac{p_{T}}{p_{z}}=\frac{p_{K^{*}} \sin \theta_{K^{*}}-p_{\gamma} \sin \theta_{\gamma}}{p_{K^{*}} \cos \theta_{K^{*}}+p_{\gamma} \cos \theta_{\gamma}}
$$

where $p_{T}$ and $p_{z}$ are the transverse and longitudinal projections of the $B$ candidate momentum and $p_{K^{*}}, p_{\gamma}, \theta_{K^{*}}$ and $\theta_{\gamma}$ are momenta and directions of its daughters in the laboratory frame. Ignoring uncertainties in all variables but the photon momentum one can estimate the error in the $B$ candidate direction as,

$$
\sigma_{\theta} \sim \frac{\sigma_{p_{\gamma}}}{p_{\gamma}}
$$

where $\frac{\sigma_{p_{\gamma}}}{p_{\gamma}}$ is the relative error in photon momentum or energy in the laboratory frame, which is about 0.013 for the $B^{0} \rightarrow K_{S}^{0} \pi^{0}$ decays and the error in $\theta$ is of the 
order of $15-20 \mathrm{mrad}$. Therefore the poor momentum and energy resolution of the $B$ candidate decay products not only makes $m_{\mathrm{ES}}$ and $\Delta E$ distributions wider, but it also introduces a substantial correlation between these two kinematic variables.

In order to improve the situation, one can try to optimize the selection of the kinematic variables by constraining the $B$ meson mass or its energy to the beam energy. In a special case of a two-body or quasi-two-body decay, when one of the $B$ meson daughters has significantly worse energy resolution than the rest with comparable angular resolution, the mass constraint or energy constraint fit can significantly improve the energy resolution as well as the resolution on the $B$ meson direction. In the case of the $B^{0} \rightarrow K_{S}^{0} \pi^{0}$ decays the energy resolution for the $K_{S}$ and $\pi^{0}$ mesons is about $20 \mathrm{MeV}$ and $60 \mathrm{MeV}$ respectively, whereas for the case of the $B^{0} \rightarrow K_{S}^{0} \pi^{0} \gamma$ the difference is significantly smaller: $K^{* 0}-40 \mathrm{MeV}$ and $\gamma-60 \mathrm{MeV}$. It was found that the gain of using a new set of kinematic variables (described below) for the $B^{0} \rightarrow K_{S}^{0} \pi^{0}$ decays, gives significant gain in the statistical significance of the signal, whereas the effect in the case of the $B^{0} \rightarrow K_{S}^{0} \pi^{0} \gamma$ was small and the standard set of kinematics variables, $m_{\mathrm{ES}}$ and $\Delta E$, has been used.

One way to make use of the $B$ mass constraint to improve the kinematic variables resolution and decrease their correlation can be realized with a set of kinematic 
variables defined as,

$$
\begin{aligned}
m_{\mathrm{rec}}^{2} & \equiv q_{B}^{2} \\
m_{\mathrm{miss}}^{2} & \equiv\left(q_{\Upsilon(4 S)}-q_{B}\left(m=M_{B}\right)\right)^{2}
\end{aligned}
$$

where $q_{B}$ and $q_{\Upsilon(4 S)}$ are 4 -momenta of the $B$ meson and the $\Upsilon(4 S)$ resonance respectively, and $q_{B}\left(m=M_{B}\right)$ is 4-momentum of the $B$ meson calculated with the mass constrained to the nominal value of the $B$ meson mass. Figure 4.9 shows the effect of using the new set of kinematic variables on the resolution and correlation of the variables for the $B^{0} \rightarrow K_{S}^{0} \pi^{0}$ decays. It shows also another set of kinematic variables, $\hat{m}$ and $\Delta E$, which was tested in the analyses. $\hat{m}$ is the $B$ meson mass calculated with a constraint $\Delta E=0$. The right plot shows a negative property of this set of kinematic variables - a significant correlation between $\Delta E$ and background shape of $\hat{m}$, expressed in term of the $\xi$ parameter of the Argus function used to parametrize the continuum background:

$$
P(\hat{m})=\hat{m} \sqrt{1-\left(\frac{\hat{m}}{m_{0}}\right)^{2}} e^{\xi\left[1-\left(\frac{\hat{m}}{m_{0}}\right)^{2}\right]} .
$$



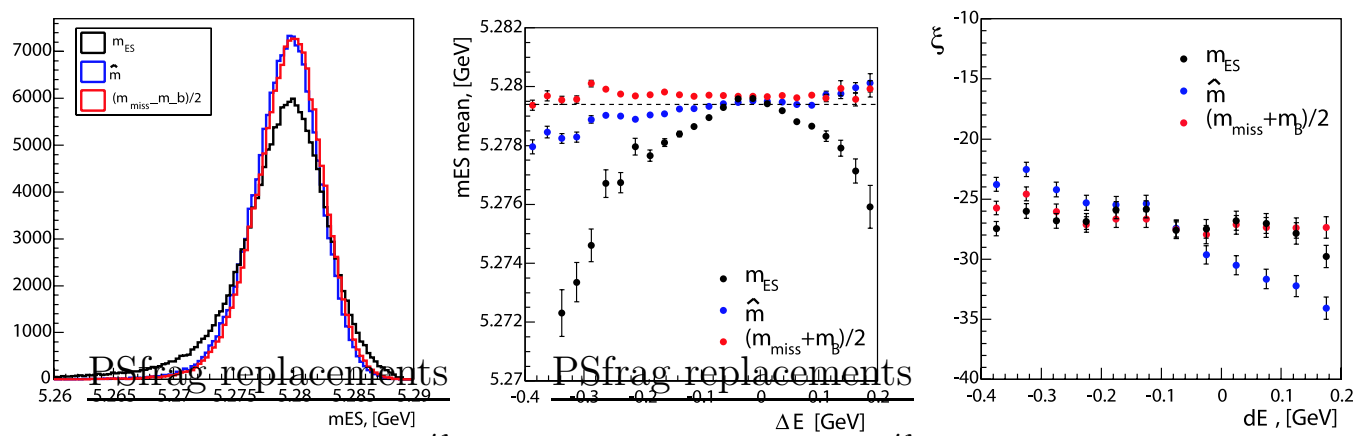

Figure 4.9: Performance comparison of different kinematic variables for the $B^{0} \rightarrow$ $K_{S}^{0} \pi^{0}$ decay. The left plot shows the improvement in the resolution for $m_{\text {miss }}$, converted to $m_{\mathrm{ES}}$ scale, with respect to the other kinematic variables. The middle plot shows smaller correlation between the kinematic variables for $m_{\text {miss }}, m_{\text {rec }}$ with respect to $m_{\mathrm{ES}}, \Delta E$. The right plot shows the correlation between background shape of $m_{\mathrm{ES}}\left(m_{\mathrm{miss}}\right)$ and $\Delta E\left(m_{\mathrm{rec}}\right)$

\subsubsection{Maximum likelihood fit}

After the final set of events is selected, the signal yield and the asymmetry information are extracted using a multidimensional maximum likelihood fit. All events are separated by the flavor tagging category and quality of the $\Delta t$ measurements: "good" events are required to satisfy $|\Delta t|<20 \mathrm{ps}$ and $\sigma_{\Delta t}<2.5 \mathrm{ps}$ conditions and events of this type are used for the time-dependent asymmetry measurements ( $S$ and $C$ terms), whereas the rest is classified as "bad" events, which enter only the time-integrated asymmetry measurements ( $C$ term). For a given category, $c$, the likelihood is defined as:

$$
\mathcal{L}_{c}=\prod_{i=1}^{N^{\text {obs }}}\left[\varepsilon_{S} \mathcal{P}_{c}^{S}\left(\vec{x}_{\text {obs }}^{i} \mid \vec{y}_{\text {param }}^{i}\right)+\left(1-\varepsilon_{S}\right) \mathcal{P}_{c}^{B}\left(\vec{x}_{\text {obs }}^{i} \mid \vec{y}_{\text {param }}^{i}\right)\right]
$$


where $\varepsilon_{S}$ is the signal fraction in the sample of events and $\mathcal{P}^{S}$ and $\mathcal{P}^{B}$ are signal and background probability distribution functions (PDFs), which have the following form for the $B^{0} \rightarrow K_{S}^{0} \pi^{0}$ analysis:

$$
\begin{aligned}
& \mathcal{P}_{c}^{S}\left(\vec{x}_{\mathrm{obs}}^{i} \mid \vec{y}_{\text {param }}^{i}\right)=\mathcal{P}^{S}\left(m_{\mathrm{rec}, i}\right) \mathcal{P}^{S}\left(m_{\mathrm{miss}, i}\right) \mathcal{P}^{S}\left(\cos \theta_{i}\right) \mathcal{P}_{c}^{S}\left(\mathcal{F}_{i}\right) \mathcal{P}_{c}^{S}\left(\Delta t_{i} \mid \sigma_{\Delta t_{i}}\right) \\
& \mathcal{P}_{c}^{B}\left(\vec{x}_{\mathrm{obs}}^{i} \mid \vec{y}_{\text {param }}^{i}\right)=\mathcal{P}^{B}\left(m_{\mathrm{rec}, i}\right) \mathcal{P}^{B}\left(m_{\mathrm{miss}, i}\right) \mathcal{P}^{B}\left(\cos \theta_{i}\right) \mathcal{P}_{c}^{B}\left(\mathcal{F}_{i}\right) \mathcal{P}_{c}^{B}\left(\Delta t_{i} \mid \sigma_{\Delta t_{i}}\right)
\end{aligned}
$$

where $\mathcal{P}^{S}\left(m_{\mathrm{rec}, i}\right), \mathcal{P}^{S}\left(m_{\text {miss }, i}\right), \mathcal{P}^{S}\left(\cos \theta_{i}\right), \mathcal{P}^{S}\left(\mathcal{F}_{i}\right), \mathcal{P}_{c}^{S}\left(\Delta t_{i} \mid \sigma_{\Delta t_{i}}\right)$ are signal PDFs of the $m_{\text {miss }}, m_{\text {rec }}$, the $B$ meson polar angle in the center of mass frame, the event shape variable and the $\Delta t$ respectively. For the $B^{0} \rightarrow K_{S}^{0} \pi^{0} \gamma$ analysis the PDFs are similar, but instead of $m_{\mathrm{rec}}$ and $m_{\mathrm{miss}}$ kinematic variables $m_{\mathrm{ES}}$ and $\Delta E$ are used and the $\mathcal{P}^{S}\left(\cos \theta_{i}\right)$ is not used in the fit. In the case of the $B^{0} \rightarrow K^{* 0} \gamma$ decay one more PDF is used in the fit, which represents the $K^{* 0}$ invariant mass.

In order to fit for the yields directly, the likelihood should be extended by the Poisson probability distribution function, which allows to take into account the fluctuations in the number of observed events of each species (signal and background for $B^{0} \rightarrow K_{S}^{0} \pi^{0}$, and signal, background and $B \bar{B}$ background for $\left.B^{0} \rightarrow K_{S}^{0} \pi^{0} \gamma\right)$.

$$
\mathcal{L}_{c}=e^{-N_{c}^{\text {pred }}} \frac{\left(N_{c}^{\text {pred }}\right)^{N_{c}^{\text {obs }}}}{N_{c}^{\text {obs } !}} \prod_{i=1}^{N_{c}^{\text {obs }}}\left[\varepsilon_{S} \mathcal{P}_{c}^{S}\left(\vec{x}_{\text {obs }}^{i} \mid \vec{y}_{\text {param }}^{i}\right)+\left(1-\varepsilon_{S}\right) \mathcal{P}_{c}^{B}\left(\vec{x}_{\text {obs }}^{i} \mid \vec{y}_{\text {param }}^{i}\right)\right]
$$

Since the fit is maximizing $\log \mathcal{L}$, multiplication or division of the likelihood by a constant has no effect on the final result. Therefore the factorial term can be dropped 
and the $\left(N_{c}^{\text {pred }}\right)^{N_{c}^{\text {obs }}}$ term can be grouped with the PDFs in the following way:

$$
\begin{aligned}
\mathcal{L}_{c}= & e^{-N_{c}^{\text {pred }}} \prod_{i=1}^{N_{c}^{\text {obs }}}\left[N_{c}^{\text {pred }} \varepsilon_{S} \mathcal{P}_{c}^{S}\left(\vec{x}_{\text {obs }}^{i} \mid \vec{y}_{\text {param }}^{i}\right)+N_{c}^{\text {pred }}\left(1-\varepsilon_{S}\right) \mathcal{P}_{c}^{B}\left(\vec{x}_{\text {obs }}^{i} \mid \vec{y}_{\text {param }}^{i}\right)\right] \\
& =e^{-\left(N_{S} \epsilon_{c}^{S}+N_{B} \epsilon_{c}^{B}\right)} \prod_{i=1}^{N_{c}^{\text {obs }}}\left[N_{S} \epsilon_{c}^{S} \mathcal{P}_{c}^{S}\left(\vec{x}_{\text {obs }}^{i} \mid \vec{y}_{\text {param }}^{i}\right)+N_{B} \epsilon_{c}^{B} \mathcal{P}_{c}^{B}\left(\vec{x}_{\text {obs }}^{i} \mid \vec{y}_{\text {param }}^{i}\right)\right]
\end{aligned}
$$

where $N_{S}$ and $N_{B}$ are the total predicted number of signal and background events, $\epsilon_{c}^{S}$ and $\epsilon_{c}^{B}$ are the fractions of events in a category $c$ for signal and background events. 


\section{Chapter 5}

\section{$B^{0} \rightarrow K_{S}^{0} \pi^{0}$ analysis}

In the previous chapter an overview was given of different aspects of a typical timedependent $C P$ violating asymmetry measurement. In this and the next chapter a detail discussion of the time-dependent asymmetry measurements is presented for the cases of the $B^{0} \rightarrow K_{S}^{0} \pi^{0}$ and $B^{0} \rightarrow K_{S}^{0} \pi^{0} \gamma$ decays. Both analyses were performed on run 1-4 data sample with the total number of $B \bar{B}$ meson pairs of $226.6 \times 10^{6}$ and $231.8 \times 10^{6}$ used for the $B^{0} \rightarrow K_{S}^{0} \pi^{0}$ and $B^{0} \rightarrow K_{S}^{0} \pi^{0} \gamma$ analyses, respectively.

\subsection{Candidate selection}

For the $B^{0} \rightarrow K_{S}^{0} \pi^{0}$ candidate reconstruction, a composite $\pi^{0}$ candidate is formed by combining two photon candidates and a pair of charged pion candidates is used to form the $K_{S} \rightarrow \pi^{+} \pi^{-}$candidates.

The photon candidates are reconstructed as neutral clusters of energy deposition in 
the EMC with the origin of the candidate set to the $B_{C P}$ meson decay vertex in order to calculate its momentum vector. The minimum energy of the photon candidate is set to $50 \mathrm{MeV}$ to reduce the amount of combinatorial and beam background. A cut on the maximum lateral moment of the energy deposition allows to separate the electromagnetic showers, which typically deposit most of their energy in just few crystals, from the hadronic showers.

The $\pi^{0}$ candidates are required to have their invariant mass within the $[110,160] \mathrm{MeV}$ range. The gain of using the merged $\pi^{0}$ candidates is negligible since the energy of the $\pi^{0}$ meson is typically low enough to be able to reconstruct the $\pi^{0}$ as a composite particle decaying to two photons.

When a pair of charged pions is combined together to form a $K_{S}$ candidate, they are fitted for a common decay vertex and the $\chi^{2}$ consistency of the fit is required to be more than 0.001 . To suppress the combinatorial background, the $K_{S}$ candidate flight length significance should be more than 5 , where the lifetime significance is defined as a ratio of the distance from the $K_{S}$ decay vertex to the $B$ meson decay vertex over the error on this distance. The invariant mass of the $K_{S}$ candidate should be within 11.2 $\mathrm{MeV}$ from the world average value. These selection criteria allow to get a list of $K_{S}$ candidates in each event that essentially has no background and almost all $K_{S}$ candidates corresponds to a real $K_{S}$ meson.

Since the $\Upsilon(4 S)$ is a vector resonance with non-zero projection of the total angular momentum along its direction in the laboratory reference frame, the angular 
distribution of the $B$ mesons in the center of mass frame depends on the angle, $\theta_{B}$, between the $B$ meson direction and the direction of the beam as,

$$
\frac{d \sigma}{d \Omega} \propto\left|Y_{J}^{M}\left(\theta_{B}, \phi\right)\right|^{2} \propto \sin ^{2} \theta_{B} .
$$

It is required that $\left|\cos \theta_{B}\right|<0.9$, which removes some background along the beam direction without significant loss in the signal efficiency.

In order to decrease the amount of the continuum background that enter the final data sample that is used for the maximum likelihood fit, the event shape parameter $L 2 / L 0$ has to be less than 0.55 . This reduces the background by a factor of 3 , with a loss of only $10 \%$ of the signal efficiency. Since the signal and background event shape variable PDFs have significant overlap, it is beneficial to use the event shape variable also in the maximum likelihood fit, which allows optimal signal extraction.

The candidate multiplicity in the final selection is $1.009 \pm 0.001$ in signal Monte Carlo sample and $1.017 \pm 0.002$ in data. When more than one candidate is found in an event, the best candidate is selected based on the $\chi^{2}$ of the $\pi^{0}$ mass with respect to the nominal value.

The total signal efficiency after the final selection and the best candidate selection is $0.341 \pm 0.014$. Corrections are applied to account for differences between Monte Carlo and data efficiency of the $\pi^{0}$ and $K_{S}$ mesons. 


\subsection{Maximum likelihood fit}

The full likelihood used in the $B^{0} \rightarrow K_{S}^{0} \pi^{0}$ analysis can be expressed as:

$$
\begin{aligned}
\mathcal{L}=e^{-\left(N_{S}+N_{B}\right)} \prod_{i \in \text { good }}^{N_{\text {good }}}\left(N_{S} f_{\text {good }}^{S} \epsilon_{c_{i}}^{S}\right. & \cdot \mathcal{P}^{S}\left(m_{\text {rec }, i}\right) \mathcal{P}^{S}\left(m_{\text {miss }, i}\right) \mathcal{P}^{S}\left(\cos \theta_{i}\right) \mathcal{P}^{S}\left(\mathcal{F}_{i}\right) P_{S}^{c}\left(\Delta t_{i}, T \mid \sigma_{\Delta t_{i}}\right)+ \\
N_{B} f_{\text {good }}^{B} \epsilon_{c_{i}}^{B} & \left.\cdot \mathcal{P}^{B}\left(m_{\text {rec }, i}\right) \mathcal{P}^{B}\left(m_{\text {miss }, i}\right) \mathcal{P}^{B}\left(\cos \theta_{i}\right) \mathcal{P}^{B}\left(\mathcal{F}_{i}\right) P_{B}^{c}\left(\Delta t_{i}, T \mid \sigma_{\Delta t_{i}}\right)\right) \times \\
\prod_{i \in \text { bad }}^{N_{\text {bad }}}\left(N_{S}\left(1-f_{\text {good }}^{S}\right) \epsilon_{c_{i}}^{S}\right. & \cdot \mathcal{P}^{S}\left(m_{\text {rec }, i}\right) \mathcal{P}^{S}\left(m_{\text {miss }, i}\right) \mathcal{P}^{S}\left(\cos \theta_{i}\right) \mathcal{P}^{S}\left(\mathcal{F}_{i}\right) \mathcal{P}_{c}^{S}(T)+ \\
N_{B}\left(1-f_{\text {good }}^{B}\right) \epsilon_{c_{i}}^{B} & \left.\cdot \mathcal{P}^{B}\left(m_{\text {rec }, i}\right) \mathcal{P}^{B}\left(m_{\text {miss }, i}\right) \mathcal{P}^{B}\left(\cos \theta_{i}\right) \mathcal{P}^{B}\left(\mathcal{F}_{i}\right) \mathcal{P}_{c}^{B}(T)\right)
\end{aligned}
$$

where $T$ is the flavor of the $B_{\text {tag }}$ candidate (for $B^{0}-T=1$ and for $\bar{B}^{0}-T=-1$ ).

The events which are flagged as having a "bad" $\Delta t$ measurement may still be tagged and used for measurement of the $C$ term of the $C P$ violating asymmetry. For signal decays the time integrated probability distribution function is:

$$
\begin{aligned}
\mathcal{P}_{c}^{S}(T) & =\int_{-\infty}^{\infty} P_{S}^{c}\left(\Delta t, T \mid \sigma_{\Delta t}\right) d(\Delta t) \\
& =\frac{1}{1-\frac{\mu_{c} C}{1+\Delta m_{d}^{2} \tau^{2}}} \cdot\left[\mu_{c} D_{c} T+\left(1+T \frac{\Delta D}{2}\right)+C \frac{T D_{c}+\mu_{c}\left(1+T \frac{\Delta D}{2}\right)}{1+\Delta m_{d}^{2} \tau_{B^{0}}^{2}}\right]
\end{aligned}
$$

where $c$ is the tagging category, $\Delta m_{d}$ is $B^{0}-\bar{B}^{0}$ mixing frequency, and $\tau_{B^{0}}$ is the $B^{0}$ lifetime. For background $\Delta t$ a similar integration yields

$$
\mathcal{P}_{c}^{B}(T)=\frac{1}{2}\left(1-T \mu_{c}^{B}\right)
$$

Figure 5.1 and figure 5.2 show the signal and background probability distribution 

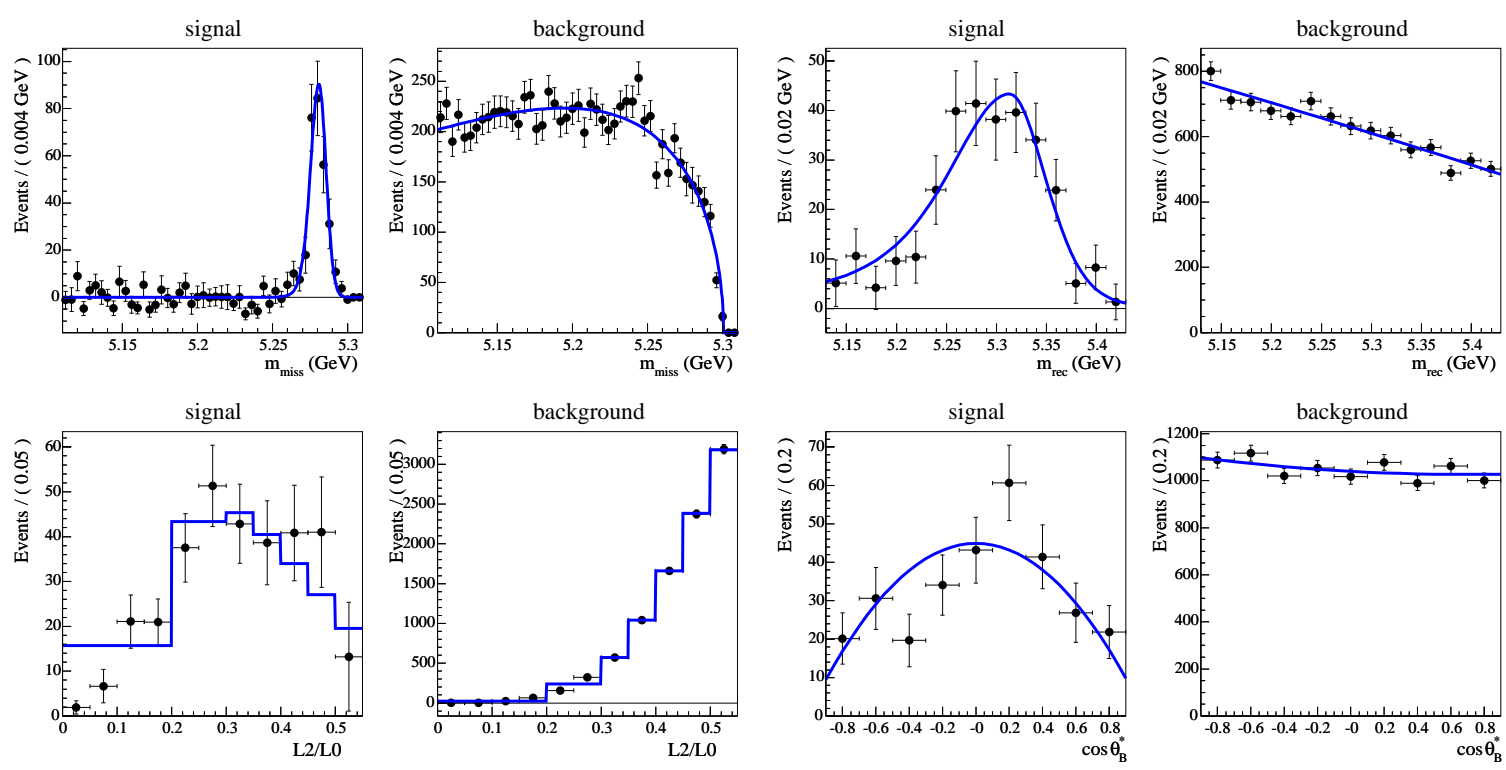

Figure 5.1: The fit model PDFs (blue curves) and background or signal subtracted data distributions (black dots) made using the s-Plot technique.

functions for different observables used in the final fit. The data distributions are made using the s-Plot technique [35], which provides a method to produce a background or signal subtracted plot without performing a series of yield fits in each bin. Since the final dataset contains a significant amount of background events, it is possible to extract all parameters of the background PDFs simultaneously from data.

In order to validate the fit procedure, several tests were performed. At first the procedure was validated using "Toy" Monte Carlo experiments in which events were generated according to the PDF parameters extracted from Monte Carlo. The Toy Monte Carlo experiments are an essential tool to identify problems in the fit procedure and calculate expectations for statistical uncertainty on the extracted measurements. However, problems associated to mistakes in the PDFs or correlations between the observables cannot be found with these tests. Therefore a set of full Monte Carlo 


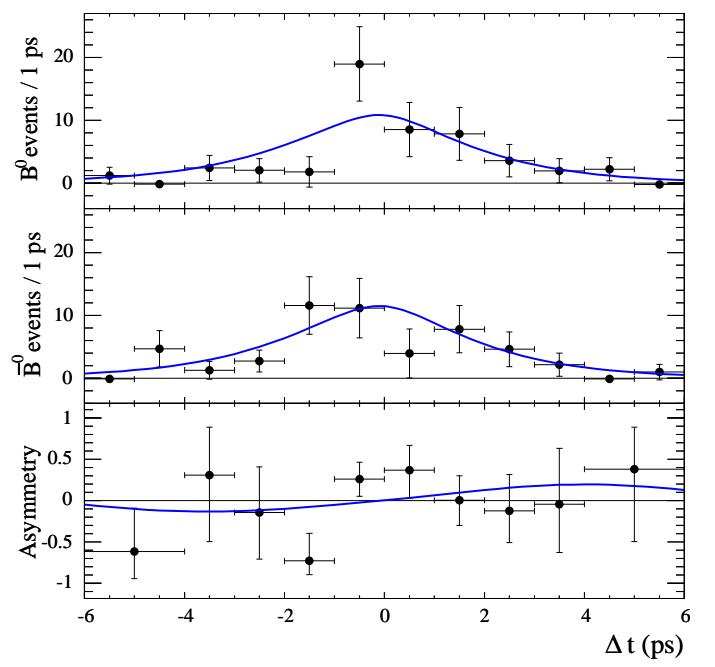

Figure 5.2: Signal s-Plots in $\Delta t$ for $B^{0}$ tags (top) and $\bar{B}^{0}$ tags (center), and the asymmetry (bottom). The solid curve represents the signal PDF extracted from data and the dashed curve is the PDF for $S=0.7$ and $C=0$.

simulations with different $C P$ violating effects were used to isolate a potential bias in the fit procedure, which was found to be negligible.

\section{$5.3 \quad B \bar{B}$ background}

The dominant source of the background in the $B^{0} \rightarrow K_{S}^{0} \pi^{0}$ decay is the continuum background that was discussed earlier. However background from the $B \bar{B}$ decays, which can mimic the signal $B$ meson decays, may also be present.

One of the special properties of the $B \bar{B}$ background, which distinguishes it from the continuum background, is the possibility of a $C P$ violating asymmetry in the background candidates. It is essential to be able to either measure this asymmetry or reliably estimate its effect on the asymmetry measurement for the signal decays. 
Using Monte Carlo simulations of a set of exclusive $B$ meson decays that could be found among the $B \bar{B}$ background events, it was found that for the current luminosity and the world average branching ratios and upper limits of the relevant $B$ meson decays the final event selection contains just few $B \bar{B}$ background events. Most of them correspond to the charged $B$ meson decays and therefore cannot have a timedependent $C P$ violating asymmetry. Most of the $B \bar{B}$ background events have $m_{\text {rec }}$ smaller than the nominal $B$ meson mass by at least $100 \mathrm{MeV}$, which makes their impact on the asymmetry measurements negligible.

\subsection{Results}

Fitting the final dataset of $9726 B^{0} \rightarrow K_{S}^{0} \pi^{0}$ candidates, it was found that the sample contains $N_{S}=300 \pm 23$ signal decays with $S_{K_{S} \pi^{0}}=0.35_{-0.33}^{+0.30}$ (stat) \pm 0.04 (syst) and $C_{K_{S} \pi^{0}}=0.06^{ \pm} 0.18$ (stat) \pm 0.03 (syst). The number of signal decays with "good" $\Delta t$ information is $f_{S}^{\text {good }} N_{S}=186 \pm 18$. Assuming equal production of charged and neutral $B$ mesons at the $\Upsilon(4 S)$ resonance, the branching ratio was found to be $\mathcal{B}\left(B^{0} \rightarrow\right.$ $\left.K_{S}^{0} \pi^{0}\right)=(11.4 \pm 0.9($ stat $) \pm 0.6($ syst $)) \times 10^{-6}$. Figure 5.3 shows the 1,2 and $3 \sigma$ contours of constant likelihood in $C-S$ plane. 


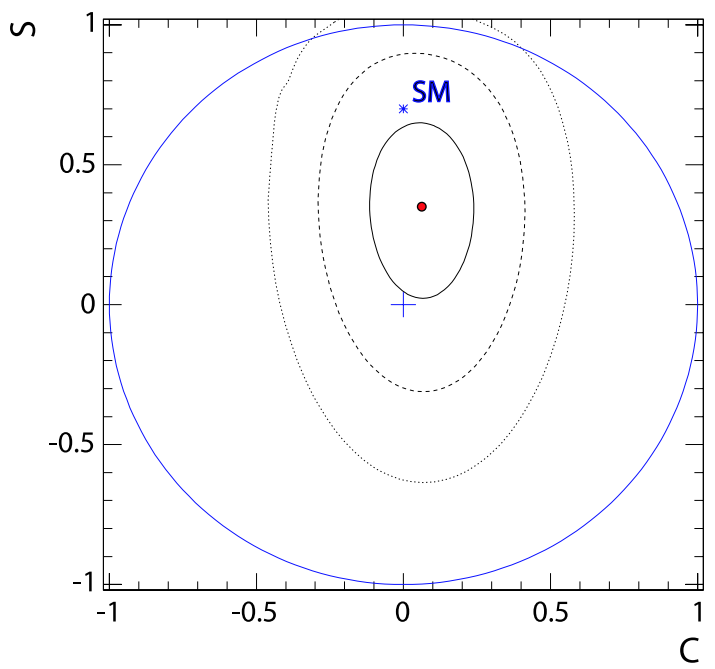

Figure 5.3: Contours of $S$ and $C$ for constant likelihood corresponding to 1, 2 and 3 standard deviations. The cross in the center is the origin $((C, S)=(0,0))$. The blue star is the Standard Model prediction $((C, S)=(0,0.7))$ and the red dot is the result of the measurement. The physical boundary is shown with a circle.

\subsection{Validations and systematic effects evaluation}

To take into account different systematic effects that can affect the final measurement, two essentially different approaches have been used. First of all the uncertainties in the input parameters of PDFs that are fixed during fitting, were evaluated by changing each parameter value by one standard deviation and observing its effect on the final measurements. Table 5.1 and table 5.2 summarize the systematic error on the asymmetry measurements and the measurement of the branching ratio.

The systematic effects due to the imperfect knowledge of the resolution function and the vertexing method used in this analysis were evaluated on a set of large signal Monte Carlo samples with different simulated asymmetries. The difference between generated and fitted asymmetries was used to assign the systematic error, which 
turned out to be quite small.

The misalignment of the DCH with respect to the SVT can lead to resolution degradation, which might affect the asymmetry measurements. To evaluate the size of this effect several sets of detector conditions were used in simulating events with different degrees of misalignment and the change in the asymmetry measurements for the large signal Monte Carlo samples was used to assign the errors.

The sensitivity to a displacement in the interaction point position is evaluated by shifting the beam position in the simulation by $\pm 20 \mu \mathrm{m}$ in the $y$ direction. The sensitivity to calibration problems or time-dependent effects is evaluated by smearing the beam-spot position by an additional $\pm 20 \mu \mathrm{m}$ in the $y$ direction. As in the case of the SVT misalignment, the change with respect to the nominal reconstruction is used to evaluate a systematic uncertainty.

The $B \bar{B}$ background contribution to the final results was evaluated using the estimated $B \bar{B}$ yield in Monte Carlo simulations, assuming that the $C P$ violating effects can get extreme values. The background subtracted s-Plots of the final data sample don't reveal any structure in the distributions of $m_{\text {rec }}$ and $m_{\text {miss }}$ that can be attributed to the $B \bar{B}$ background, which exclude a possibility of significant $B \bar{B}$ underestimation in the Monte Carlo simulations.

In the maximum likelihood fit, the flavor tagging asymmetry in background events was accounted for by floating the asymmetry parameter for each tag category. This asymmetry can arise due to small charge asymmetry of the tracking and particle 
identification efficiencies as well as the tagging algorithm asymmetries in the neural net training. The systematic effect was estimated by comparing the nominal fit results with a fit, where all the asymmetry parameters were set to zero.

The $B^{0}$ meson lifetime and mixing frequency used in this analysis were taken from the Review of Particle Physics 2004 [36]. The uncertainty in these parameters were used to evaluate the corresponding systematic errors.

The systematic uncertainties in the branching ratio measurement are greatly affected by the uncertainty in the PDFs parameterization and event selection cuts. The results of dedicated efficiency studies for the $K_{S}$ and $\pi^{0}$ were used to properly evaluate the effect of each selection cut. Several control samples of exclusively reconstructed decays were used for comparison tests between data and Monte Carlo simulations. A sample of $B^{+} \rightarrow X^{+} \pi^{0}$ decays, where $X$ is any charged particle, was used to estimate the systematic uncertainty on the event shape variable selection cut. A sample of $B^{+} \rightarrow \pi^{+} \pi^{0}$ decays was used for $m_{\text {rec }}$ efficiency calculations.

In order to evaluate the effect of possible correlation of the observables in signal, two different samples of full Monte Carlo simulated events were mixed with background events, which were generated according to the nominal PDF parameterization used in the final fit. The first sample corresponding to August 2001 detector conditions provided enough data to perform about 400 independent experiments, which revealed a small bias on the signal yield, if only yields, $S$ and $C$ were floated in the fit. The bias can be completely eliminated by floating signal $m_{\text {rec }}$ mean and signal 
flavor tagging fractions. The other sample was optimized to simulate data as close as possible. It included the neutral efficiency and energy corrections and a proper mix of different conditions for RUN1-4. It allowed to perform 172 independent experiments and no bias were found.

To summarize, the systematic uncertainties in $S$ and $C$ are not dominated by a single source. For the measurement of $S$, the SVT alignment makes an appreciable contribution. With more statistics the uncertainty due to the $B \bar{B}$ background can be reduced. The uncertainty due to the resolution function originates in data/Monte Carlo differences of the width of the $\Delta t$ mangled/nominal pull distributions. The uncertainty in the branching fraction is not dominated by a single source. Improvement in the understanding of the efficiency and energy scale of the calorimeter will improve the uncertainty on the efficiency of the $\pi^{0}$ selection and the $m_{\text {rec }}$ selection and parameterization. The uncertainty in the $K_{S}^{0}$ efficiency can be reduced slightly by removing the $\chi^{2}$ cuts.

\subsection{Summary}

In this chapter the result of the time-dependent $C P$ violating asymmetry measurement was presented and it was found that

$$
\begin{aligned}
& S_{K_{S}^{0} \pi^{0}}=0.35_{-0.33}^{+0.30}(\text { stat }) \pm 0.04(\text { syst }) \\
& C_{K_{S}^{0} \pi^{0}}=0.06 \pm 0.18 \text { (stat) } \pm 0.03 \text { (syst) } .
\end{aligned}
$$




\begin{tabular}{l|cccc}
\hline \hline & \multicolumn{3}{|c}{$\Delta S$} & \multicolumn{2}{c}{$\Delta C$} \\
& $(+)$ & $(-)$ & $(+)$ & $(-)$ \\
\hline$m_{\text {miss pdf }}$ & 0.0011 & -0.0025 & 0.0021 & -0.0024 \\
$m_{\text {rec pdf }}$ & 0.0018 & -0.0026 & 0.0012 & -0.0014 \\
$l_{2}$ pdf & 0.0045 & -0.0078 & 0.0050 & -0.0047 \\
cos $\theta_{\text {CM pdf }}$ & 0.0007 & -0.0001 & 0.0003 & -0.0003 \\
resolution function & 0.0041 & -0.0059 & 0.0030 & -0.0029 \\
Dilution and $\Delta D$ & 0.0051 & -0.0062 & 0.0102 & -0.0102 \\
$\epsilon_{\text {tag }}$ & 0.0005 & -0.0011 & 0.0007 & -0.0006 \\
$\mu^{S}$ & 0.0029 & -0.0046 & 0.0141 & -0.0139 \\
\hline pdf total & 0.012 & 0.018 \\
beam-spot & 0.004 & 0.001 \\
SVT Alignment & 0.020 & 0.007 \\
data/MC RF & 0.014 & 0.004 \\
vertexing method & 0.018 & 0.013 \\
$B \bar{B}$ backgrounds & 0.019 & 0.015 \\
$\tau_{B}$ and $\Delta m_{d}$ & 0.005 & 0.005 \\
tag side interference & 0.003 & 0.014 \\
\hline Total & 0.038 & 0.031 \\
\hline \hline
\end{tabular}

Table 5.1: Breakdown of all contributions to the systematic uncertainty on $S$ and $C$.

\begin{tabular}{l|cc}
\hline \hline$m_{\text {miss }}$ pdf & +0.013 & -0.011 \\
$m_{\text {rec }}$ pdf & +0.003 & -0.002 \\
$l_{2}$ pdf & +0.014 & -0.014 \\
$\cos \theta_{\mathrm{CM}}$ pdf & +0.001 & -0.001 \\
Dilution and $\Delta D$ & +0.001 & -0.001 \\
$\epsilon_{\text {tag }}$ & +0.003 & -0.001 \\
$\mu^{S}$ & +0.001 & -0.001 \\
\hline pdf total & 0.020 \\
$B \bar{B}$ backgrounds & 0.014 \\
$K_{S}^{0}$ selection & 0.026 \\
$\pi^{0}$ selection & 0.030 \\
$m_{\text {rec selection }}$ selion & 0.020 \\
$l_{2}$ selection & 0.012 \\
fit bias & 0.008 \\
$B$ counting & 0.011 \\
\hline total & 0.053 \\
\hline \hline
\end{tabular}

Table 5.2: Breakdown of all contributions to the (multiplicative) systematic uncertainty on the branching fraction. 
It was also found that the branching ratio of the $B^{0} \rightarrow K_{S}^{0} \pi^{0}$ decay is:

$$
\mathcal{B}\left(B^{0} \rightarrow K_{S}^{0} \pi^{0}\right)=(11.4 \pm 0.9(\text { stat }) \pm 0.6(\text { syst })) \times 10^{-6}
$$

These results are statistics limited and the systematic uncertainties will improve with increase in the data sample size. 


\section{Chapter 6}

\section{$B^{0} \rightarrow K_{S}^{0} \pi^{0} \gamma$ analysis}

The time-dependent asymmetry measurements in the $B^{0} \rightarrow K_{S}^{0} \pi^{0} \gamma$ decay closely follows the measurements in the $B^{0} \rightarrow K_{S}^{0} \pi^{0}$ decay, even though the physics, as it was discussed in the theory chapter, is different. While in the $B^{0} \rightarrow K_{S}^{0} \pi^{0}$ case $C P$ violation is expected and predicted by the Standard Model, in the $B^{0} \rightarrow K_{S}^{0} \pi^{0} \gamma$ case observation of significant $C P$ violating asymmetry would clearly indicate the presence of new physics.

One important aspect of the reconstruction of the $B^{0} \rightarrow K_{S}^{0} \pi^{0} \gamma$ decay, which distinguishes it from the $B^{0} \rightarrow K_{S}^{0} \pi^{0}$ decay, lies in the existence of a rich resonance structure found in the 3-body decays. 


\subsection{The effect of resonances on the $C P$ violating observables}

The sign of the $S$ term of the time-dependent $C P$ violation is determined by the $C P$ eigenvalue of the final state. In the case of a 3-body decay the asymmetry measurement can be complicated by the presence of resonances, which can represent final states with different $C P$ eigenvalues and effectively dilute the asymmetry. Recently it was shown [38] that in $B \rightarrow P_{1} P_{2} \gamma$ case, where $P_{1}$ and $P_{2}$ are two pseudo-scalers, the sign of the asymmetry can be calculated using just the $C$-eigenvalue of the $P_{1}-P_{2}$ system, which effectively makes the sign of the asymmetry independent of the resonance structure if $P_{1}$ and $P_{2}$ are two different pseudo-scalers as it is in the $B^{0} \rightarrow K_{S}^{0} \pi^{0} \gamma$ decay.

If we neglect the small $C P$ violation effect in the neutral kaons, we can deduce that $K_{S}$ has the following quantum numbers: $J^{P C}=0^{--}\left(K_{S}\right.$ decays to the $C P$-even state and it is a pseudo-scalar meson). Taking into account that $\pi^{0}$ is $0^{-+}$state we

find that $\eta_{C}\left(K_{S} \pi^{0}\right)=-1$ and it doesn't depend on the relative orbital momentum of $K_{S}-\pi^{0}$ system.

The result would be different if we would consider $\pi^{+} \pi^{-} \gamma$ final state, since $C$-parity of $\pi^{+} \pi^{-}$system depends on the relative orbital angular momentum of the two pions. This follows from the "generalized" Pauli principle [34], which states that the wavefunction of a particle-antiparticle state is symmetric under exchange of two mesons. 
The symmetry of the state is $(-1)^{L}(-1)^{S} C$ and should be 1 , therefore $C=(-1)^{L+S}$. So we come to the conclusion that in the case of $\pi^{+} \pi^{-} \gamma$ the resonance structure does matter and the sign of the $S$-term of the time-dependent $C P$ violation depends on the relative orbital angular momentum of the two pions.

It is possible to show from the first principles that in the case of $B^{0} \rightarrow K_{S}^{0} \pi^{0} \gamma$ the $C P$ eigenvalue of the final state in fact doesn't depend on the resonance structure. The $C P$ eigenvalue of the final state can be expressed as:

$$
\eta_{C P}=(-1)^{L_{X \gamma}}(-1)^{L_{X}} \eta_{C}=(-1)^{L_{X \gamma}+L_{X}+1}
$$

where $L_{X \gamma}$ and $L_{X}$ are the relative orbital angular momenta of the $\left(K_{S} \pi^{0}\right)-\gamma$ and $K_{S}-\pi^{0}$ systems respectively $\left(J_{X}=L_{X}\right.$ since $X$ decays to two pseudo-scalers). It is convenient to choose the quantization axis to be the photon direction in the $B$ rest frame. In this case the projection of the relative orbital angular momentum of the $X-\gamma$ system is always zero. To see how $L_{X \gamma}$ and $J_{X}$ are related let us choose some specific photon polarization that allows the time-dependent asymmetry to be observed, i.e. the photon is either parallel or perpendicular polarized in the transversity basis:

$$
\left|\gamma_{\|, \perp}\right\rangle=\frac{1}{\sqrt{2}}(|R\rangle \pm|L\rangle)=\frac{1}{\sqrt{2}}\left(\left|S_{\gamma}, m_{\gamma}=+1\right\rangle \pm\left|S_{\gamma}, m_{\gamma}=-1\right\rangle\right)
$$


The $B$ meson is a pseudo-scaler and therefore:

$$
|B\rangle=|0,0\rangle=\left|S_{X \gamma}, m_{S}\right\rangle \otimes\left|L_{X \gamma}, m_{L}\right\rangle=\left|S_{X \gamma}, 0\right\rangle \otimes\left|L_{X \gamma}, 0\right\rangle .
$$

According to the triangular condition of the angular momentum addition:

$$
\left|J_{1}-J_{2}\right| \leq J \leq\left|J_{1}+J_{2}\right|
$$

and therefore $S_{X \gamma}=L_{X \gamma}$. So the problem is reduced to showing how the total spin of $X-\gamma$ system is related to the total angular momentum of $X$. Using the general form of the Clebsch-Gordan coefficients (Wigner (1931), Racah (1942)) it is easy to show that:

$$
\left\langle j_{1} j_{2} m_{1} m_{2} \mid j_{1} j_{2} J M\right\rangle=(-1)^{J-j_{1}-j_{2}}\left\langle j_{1} j_{2}-m_{1}-m_{2} \mid j_{1} j_{2} J M\right\rangle
$$

which means that the Clebsch-Gordan coefficient doesn't change or change sign under $m_{1} \rightarrow-m_{1}$ and $m_{2} \rightarrow-m_{2}$ simultaneous transformation. Now we can deduce the selection rules that relate $L_{X \gamma}$ and $L_{X}$. Using equation 6.5 one gets:

$$
\begin{aligned}
\left\langle L_{X \gamma} 0 \mid \gamma_{\|, \perp}\right\rangle=\frac{1}{\sqrt{2}}\left\langle L_{X \gamma} 0 \mid J_{X} S_{\gamma} m_{X} m_{\gamma}\right\rangle & \left(1 \pm(-1)^{L_{X \gamma}-S_{\gamma}-J_{X}}\right) \\
\propto & \left(1 \pm(-1)^{L_{X \gamma}-J_{X}-1}\right) .
\end{aligned}
$$

Therefore $L_{X \gamma}+L_{X}$ should be odd if the photon has parallel polarization (linear 
polarization in the plane defined by the $K_{S}^{0}$ and $\pi^{0}$ momenta) and even in the case of the perpendicular polarization (linear polarization in the plane perpendicular to the plane defined by the $K_{S}^{0}$ and $\pi^{0}$ momenta), which depends on the type of the new physics contributing to the decay amplitude. This leads to the conclusion that $C P$ eigenvalue of the final state $B^{0} \rightarrow K_{S}^{0} \pi^{0} \gamma$ doesn't depend on the resonance structure of the decay and is defined by the photon polarization.

\subsection{Candidate selection}

The $B^{0} \rightarrow K_{S}^{0} \pi^{0} \gamma$ candidate reconstruction is similar to the $B^{0} \rightarrow K_{S}^{0} \pi^{0}$ candidate reconstruction with one extra photon in the final state. Given that the $S$-wave state of the $K_{S}-\pi^{0}$ system is not allowed due to the conservation of the angular momentum and the fact that the $K^{* 0}$ resonance is well separated from higher order resonances, the data sample was split in two parts. Events with invariant mass $m_{K_{S} \pi^{0}} \in[0.8,1.0] \mathrm{GeV}$ were used for the analysis of resonant $B^{0} \rightarrow K^{* 0} \gamma$ decays and events with $m_{K_{S} \pi^{0}} \in$ $[1.1,1.8] \mathrm{GeV}$ entered the analysis of the 3-body mode. This choice was supported by a recent measurement of the mass distribution in $b \rightarrow s \gamma$ semi-inclusive decays [32], shown in figure 6.1. This result shows that the mass spectrum is effectively separated into two regions. In the $K^{* 0}$ region $\left(m_{X} \in[0.8,1.0] \mathrm{GeV}\right)$ the line-shape is well known and can be parameterized with a Breit-Wigner distribution. In the non- $K^{*}$ region $m_{X} \in[1.1,1.8] \mathrm{GeV}$ the line shape is not well known: theoretical predictions, such as from the Kagan-Neubert model [40], have significant theoretical uncertainties and do 


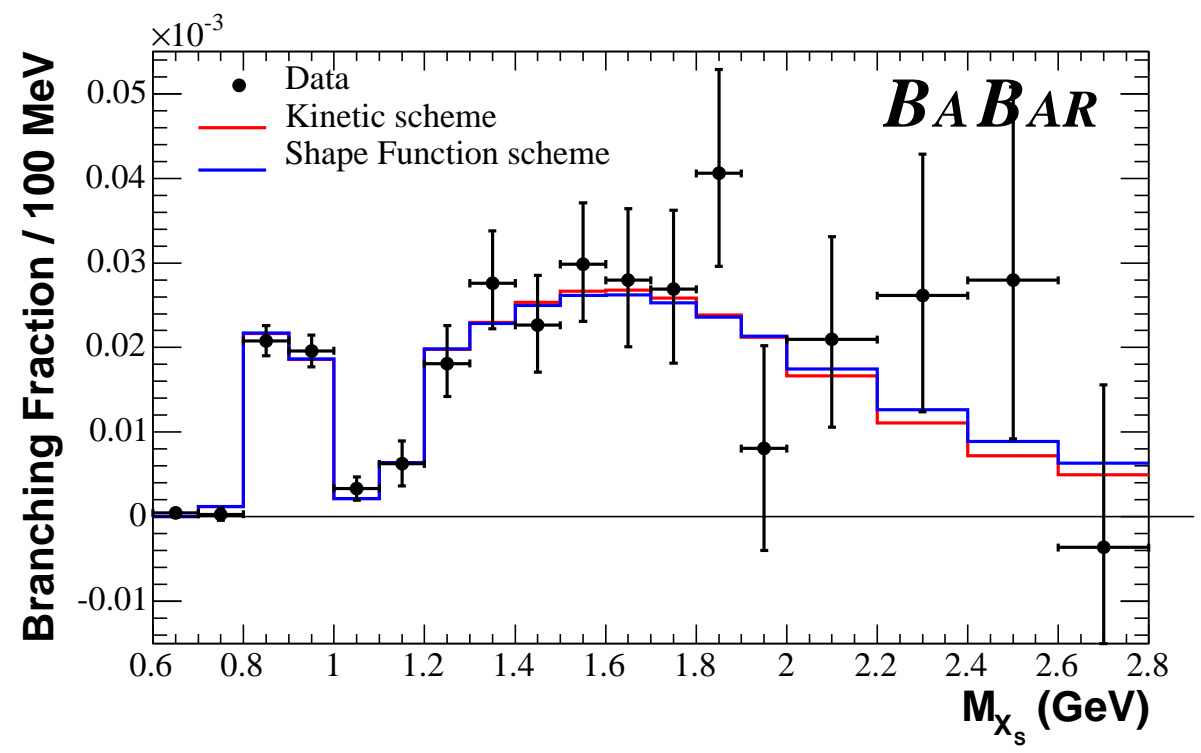

Figure 6.1: The hadronic mass spectrum of the semi-inclusive $b \rightarrow s \gamma$ decays. The data points are compared to theoretical predictions using different theoretical models.

not include resonances such as $K_{2}^{*}(1430)$. Furthermore, the contribution from higher order resonances is not well known. Consequently, the $K_{S}^{0} \pi^{0}$ invariant mass was not exploited in the fit for the 3-body mode and regions of the mass spectrum with low expected ratio of signal over background were not used. Events with $m_{X}>1.8 \mathrm{GeV}$ were excluded, since the region $[1.8,2.0] \mathrm{GeV}$ receives a contribution from background events with a $D^{0} \rightarrow K_{S} \pi^{0}$ decay and the contribution from events with $m_{X}>2.0 \mathrm{GeV}$ is small since the 2-body hadronic final states have harder photon spectrum and therefore lower invariant mass of the $K_{S} \pi^{0}$ system than the inclusive spectrum.

The photon candidate that originates from the $B$ meson decay directly is quite energetic and can be reliably reconstructed with few quality and isolation criteria. The candidate energy deposition was required to be isolated from other energy depositions by at least $25 \mathrm{~cm}$ at the EMC. It was also required that the photon candidate should 

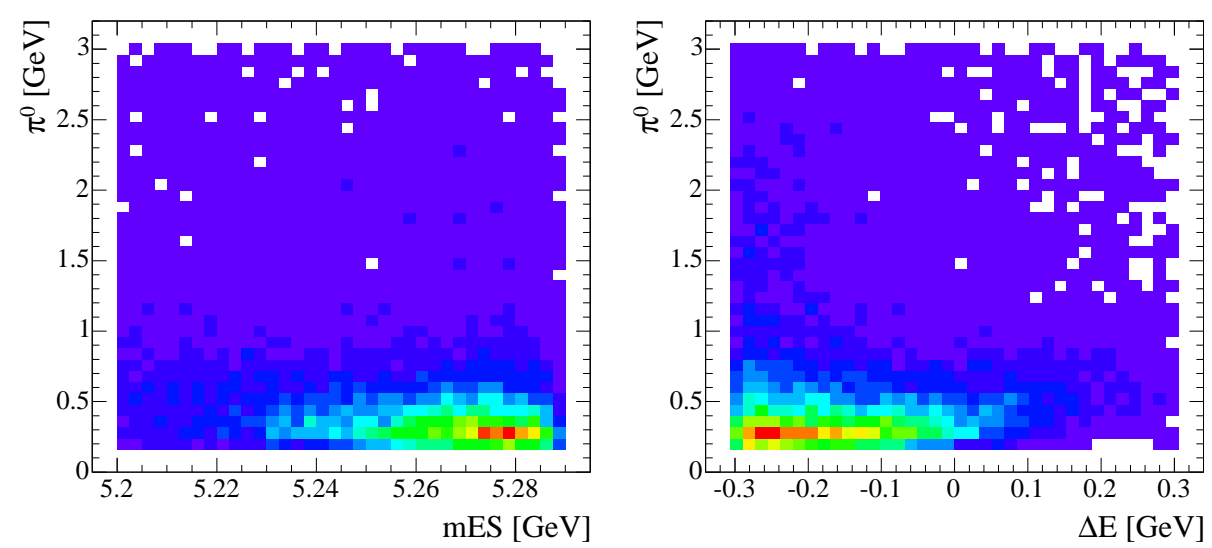

Figure 6.2: $B^{+} B^{-}$background distributions of $\Delta E$ and $m_{\mathrm{ES}}$ versus $\pi^{0}$ energy.

not form a $\pi^{0}$ or $\eta$ candidate with any other photon in the event. The veto $\pi^{0}$ and $\eta$ are required to satisfy tight reconstruction criteria: the minimum energies of the photon candidates are $50 \mathrm{MeV}$ and $250 \mathrm{MeV}$ for $\pi^{0}$ and $\eta$, respectively, and the invariant mass of $\eta$ is in the $[0.470,0.620] \mathrm{GeV}$ range. Since a high energy $\pi^{0}$ can look like a photon in the EMC, a cut on the lateral energy distribution of the EMC cluster was used to suppress this type of fake photon candidates.

For the $\pi^{0}$ candidate selection a requirement on the minimum $\pi^{0}$ energy in the laboratory reference frame was set to $0.59 \mathrm{GeV}$. This requirement is effective in reducing the fraction of wrong and fake $\pi^{0}$ and $K^{* 0}$ candidates, both from continuum and from $B \bar{B}$ background. Figure 6.2 shows how $B^{+} B^{-}$background yield depends on the $\pi^{0}$ energy.

Since the $K^{* 0}$ meson can only have non-zero spin projection along its momentum in the $B$ meson rest frame when the $B$ meson decays to $K^{*} \gamma$ final state, the angular 
distribution of the $K^{* 0}$ decay products is expected to be:

$$
\frac{d \sigma}{d \Omega} \propto\left|Y_{J}^{M}\left(\theta_{H}, \phi\right)\right|^{2} \propto \sin ^{2} \theta_{H}
$$

where $\theta_{H}$ is the angle between the $K_{S}$ direction and the $K^{* 0}$ direction in the $K^{* 0}$ rest frame. It was required that the candidates with the invariant mass of $K_{S} \pi^{0}$ in the $K^{* 0}$ region $([0.8,1.0] \mathrm{GeV})$ satisfied $\left|\cos \theta_{H}\right|<0.9$ condition.

The following list summarizes the final candidate selection requirements used in the analysis.

- photon selection

- energy in the laboratory frame is more than $30 \mathrm{MeV}$

- energy in the center of mass frame is in $[1.5,3.5] \mathrm{GeV}$

- the lateral energy distribution, $\mathrm{LAT} \in[0.0: 0.8]$

$-E_{\mathrm{CMS}} \in[1.5,3.5] \mathrm{GeV}$

- second cluster moment (scaled to $z=0) S_{z=0}<0.0022$.

- distance to closest other (charged or neutral) EMC cluster $<25 \mathrm{~cm}$

$-\pi^{0}$ and $\eta$ veto

- $\pi^{0} \rightarrow \gamma \gamma$ selection

- photon energy in the laboratory frame is more than $30 \mathrm{MeV}$

- the lateral energy distribution of each photon, LAT $\in[0.0: 0.8]$ 
$-m_{\pi^{0}} \in[0.115: 0.155] \mathrm{GeV}$

- $\pi^{0}$ energy in the laboratory frame is more than $0.59 \mathrm{GeV}$

- $K_{S}^{0} \rightarrow \pi^{+} \pi^{-}$selection

$-m_{K_{S}^{0}} \in[0.487 ; 0.508] \mathrm{GeV}$

- flight length significance $>5$

- vertex $\chi^{2}$ consistence $>0.001$

- $K^{* 0} \rightarrow K_{S}^{0} \pi^{0}$ selection

$-0.8<m_{K_{S}^{0} \pi^{0}}<1.0 \mathrm{GeV}$

$-\left|\cos \theta_{H}\right|<0.9$

- $B^{0} \rightarrow K^{* 0} \gamma$ and $B^{0} \rightarrow K_{S}^{0} \pi^{0} \gamma$ selection

$-m_{\mathrm{ES}} \in[5.2: 5.3] \mathrm{GeV}$

$-|\Delta E|<0.25 \mathrm{GeV}$

$-\left|\cos \theta_{B}\right|<0.9$

After all cuts are applied the main source of multiple candidates is fake $\pi^{0}$ candidates. The best $B$ meson candidate is selected based on the $\chi^{2}$ of the reconstructed $K_{S}$ and $\pi^{0}$ mass,

$$
\chi^{2}=\left(\frac{m_{K_{S}^{0}}-m_{K_{S}^{0}}^{\mathrm{pdg}}}{\sigma_{m_{K_{S}^{0}}}}\right)^{2}+\left(\frac{m_{\pi^{0}}-m_{\pi^{0}}^{\mathrm{pdg}}}{\sigma_{m_{\pi^{0}}}}\right)^{2} .
$$


The average $B^{0} \rightarrow K^{* 0} \gamma$ candidate multiplicity was found to be 1.22 in Monte Carlo and 1.17 in data. The best candidate selection is about $94 \%$ efficient on a sample of Monte Carlo generated events, where at least one $B$ meson candidate was truth matched to be correctly reconstructed. In the case of 3-body $B^{0} \rightarrow K_{S}^{0} \pi^{0} \gamma$ decays, the candidate multiplicity was found to be smaller at the level of 1.08 and the best candidate selection was about $97 \%$ efficient. This difference is coming from the fact that the $\pi^{0}$ on average is more energetic in the 3-body decay.

The overall final candidate selection efficiency was estimated to be $0.1649 \pm 0.0005$ using only signal Monte Carlo simulated events. The reconstruction efficiency for the 3-body decay $B^{0} \rightarrow K_{S}^{0} \pi^{0} \gamma$ has only a mild dependence on the $K_{S} \pi^{0}$ invariant mass up to approximately $3 \mathrm{GeV}$. The cutoff is determined by the photon energy threshold of $1.5 \mathrm{GeV}$ in the center of mass frame.

\section{3 $B \bar{B}$ background}

In contrast to the $B^{0} \rightarrow K_{S}^{0} \pi^{0}$ decay, where the $B \bar{B}$ background is essentially negligible, in the case of the $B^{0} \rightarrow K_{S}^{0} \pi^{0} \gamma$ decay it represents a significant problem. The reason for this is the relatively high decay rate of the inclusive $b \rightarrow s \gamma\left(B \rightarrow X_{S} \gamma\right)$ decays. The photon and $K_{S}$ mesons can be reliably reconstructed in most cases, but poor energy resolution limits the separating power of the kinematic variables. The most detrimental $B \bar{B}$ background corresponds to the candidates with low energy $\pi^{0} \mathrm{~s}$, because in an asymmetric decay a lower energy pion can be replaced with another one 
and the $B$ meson candidate will still look like the signal. For example a $B^{+} \rightarrow K_{S} \pi^{+} \gamma$ decay can be reconstructed as $B^{0} \rightarrow K_{S}^{0} \pi^{0} \gamma$ and look like signal in $m_{\mathrm{ES}}$ and $\Delta E$ if the pion energy is low. This is the motivation for a tight cut on the minimum $\pi^{0}$ energy in the laboratory frame. This cut removes most of the peaking signal-like $B \bar{B}$ background candidates.

The $B \bar{B}$ background evaluation is complicated by poor knowledge of the photon spectrum in the inclusive $B \rightarrow X_{S} \gamma$ decays. Theoretical models [40] can reliably predict only the first few moments of the photon energy distribution. The resonance structure of the decay is essentially unknown except for few experimentally measured exclusive resonant decays. This limitation is coming from high uncertainty in the long distance effects in the QCD, which control the resonance properties of the $B \rightarrow X_{S} \gamma$ decay. The fragmentation of the $s$-quark is also poorly simulated in the Monte Carlo. To overcome these difficulties different energy distributions were used as a cross check and the fragmentation of the $s$-quark in Monte Carlo was corrected using the results of the semi-inclusive $B \rightarrow X_{S} \gamma$ measurement.

Table 6.1 shows the expected $B \bar{B}$ background yield using Monte Carlo simulated events with the fragmentation corrections applied. Table 6.2 and table 6.3 show the content of the $B \bar{B}$ background after final event selection on a sample of Monte Carlo simulated events corresponding to roughly 4 times larger data set than the one used for the measurement. An extensive study of the background content during optimization of the candidate selection, allowed to suppress most of the dominant sources of the 


\begin{tabular}{lcc}
\hline \hline & $B^{0} \rightarrow K^{* 0} \gamma$ & $B^{0} \rightarrow K_{S}^{0} \pi^{0} \gamma$ \\
\hline$B^{0} B^{0}$, excluding $B \rightarrow X_{d s} \gamma$ & $7.6 \pm 1.4$ & $41.6 \pm 3.3$ \\
$B^{0} \rightarrow X_{S}^{0} \gamma$, excluding $B^{0} \rightarrow K_{S}^{0} \pi^{0} \gamma$ & $12.4 \pm 1.9$ & $33.3 \pm 3.8$ \\
$B^{+} B^{-}$, excluding $B \rightarrow X_{S} \gamma$, including $B^{ \pm} \rightarrow K^{* \pm} \gamma$ & $10.2 \pm 1.6$ & $59.5 \pm 3.9$ \\
$B^{+} \rightarrow X_{u s}^{+} \gamma$ & $14.0 \pm 2.3$ & $35.2 \pm 4.6$ \\
\hline total neutral & $20.0 \pm 2.4$ & $74.9 \pm 5.0$ \\
total charged & $24.2 \pm 2.8$ & $94.7 \pm 6.0$ \\
\hline \hline
\end{tabular}

Table 6.1: The expected event yields for different $B \bar{B}$ background contributions after the final selection with the fragmentation corrections applied

background. The remaining $B \bar{B}$ background events correspond to several different physics processes. The $B^{+} \rightarrow K_{S} \pi^{+} \gamma$ decay represents about $40 \%$ of the $B^{+} B^{-}$ background. In principle it is possible to suppress this source of background by explicitly reconstructing this decay, which is planned to be done in a future update of this measurement when a larger data sample becomes available.

\subsection{The maximum likelihood fit}

The maximum likelihood fit used for the $B^{0} \rightarrow K_{S}^{0} \pi^{0} \gamma$ analysis is identical to that used for the $B^{0} \rightarrow K_{S}^{0} \pi^{0}$ analysis, except for the following modifications:

- the Tag04 flavor tagger was used instead of the Moriond flavor tagger. The new tagger has slightly higher effective tagging efficiency (30.5 \pm 0.6 versus $29.3 \pm 0.6)$.

- $m_{\mathrm{ES}}$ and $\Delta E$ kinematic variables were used instead of $m_{\text {miss }}$ and $m_{\mathrm{rec}}$. New kinematic variables $\left(m_{\text {rec }}\right.$ and $\left.m_{\text {miss }}\right)$ didn't show significant gain with respect to the standard set of variables. 


\begin{tabular}{lcc}
\hline \hline mode & $B^{0} \rightarrow K^{* 0} \gamma$ & $B^{0} \rightarrow K_{S}^{0} \pi^{0} \gamma$ \\
\hline$K_{S}^{0} \pi^{0} \pi^{0} \gamma$ & 17 & 20 \\
$K_{S}^{0} \pi^{+} \pi^{-} \gamma$ & 4 & 27 \\
$K_{S}^{0} \eta \gamma$ & 8 & 13 \\
$K_{S}^{0} \gamma \gamma$ & 2 & 0 \\
$K_{L}^{0} \pi^{0} \gamma$ & 0 & 17 \\
$K^{+} \pi^{-} \pi^{0} \gamma$ & 0 & 10 \\
$\pi^{0} \pi^{0} \pi^{0} \gamma$ & 0 & 3 \\
$\pi^{0} \pi^{0} \pi^{-} K^{+} \gamma$ & 0 & 3 \\
$K_{S}^{0} \pi^{-} \pi^{+} \pi^{0} \gamma$ & 0 & 6 \\
$K_{S}^{0} \pi^{0} \pi^{+} \pi^{0} \gamma$ & 0 & 4 \\
$K_{S}^{0} \eta^{\prime} \gamma\left(\eta^{\prime} \rightarrow \gamma \gamma\right)$ & 0 & 2 \\
$K_{S}^{0} \pi^{0} \eta$ & 10 & 7 \\
$K_{S}^{0} \eta^{\prime}\left(\eta^{\prime} \rightarrow \gamma \gamma\right)$ & 0 & 8 \\
$K_{S}^{0} \pi^{0} \pi^{0}$ & 2 & 7 \\
$\pi^{0} \pi^{0}$ & 0 & 2 \\
$K_{S}^{0} \pi^{0} \pi^{+} \pi^{0}$ & 0 & 2 \\
$\pi^{0} \pi^{0} \pi^{-} \pi^{+}$ & 0 & 2 \\
$\pi^{0} \pi^{0} \pi^{0} \pi^{-} \pi^{+}$ & 0 & 2 \\
other & 30 & 149 \\
\hline total & 73 & 284 \\
\hline \hline
\end{tabular}

Table 6.2: Final state topologies contributing to the $B^{0} \rightarrow K^{* 0} \gamma$ and $B^{0} \rightarrow K_{S}^{0} \pi^{0} \gamma$ final states originating from $B^{0} \bar{B}^{0}$ decays. Numerical estimates are given for Monte Carlo data sets of $799 / \mathrm{fb}$. Only topologies appearing more than once are shown. (The remaining contribution is called 'other'.) 


\begin{tabular}{lcc}
\hline \hline & $B^{0} \rightarrow K^{* 0} \gamma$ & $B^{0} \rightarrow K_{S}^{0} \pi^{0} \gamma$ \\
\hline$K^{*+} \gamma\left(K^{*+} \rightarrow K_{S}^{0} \pi^{+}\right)$ & 21 & 114 \\
other $K_{S}^{0} \pi^{+} \gamma$ & 15 & 68 \\
$K_{S}^{0} \pi^{+} \pi^{0} \gamma$ & 34 & 66 \\
$K_{S}^{0} \pi^{+} \pi^{+} \pi^{-} \gamma$ & 0 & 6 \\
$K^{+} \pi^{0} \gamma$ & 2 & 25 \\
$K_{S}^{0} \pi^{+} \pi^{0} \pi^{0}$ & 0 & 12 \\
$K_{S}^{0} \pi^{0} \pi^{0} \pi^{-}$ & 0 & 6 \\
$K_{S}^{0} \pi^{+} \eta$ & 2 & 7 \\
$\pi^{+} \pi^{0} \pi^{0}$ & 0 & 14 \\
$\pi^{+} \pi^{0} \pi^{0} \pi^{0}$ & 0 & 3 \\
other & 16 & 84 \\
\hline total & 90 & 405 \\
\hline \hline
\end{tabular}

Table 6.3: Final state topologies contributing to the $B^{0} \rightarrow K^{* 0} \gamma$ and $B^{0} \rightarrow K_{S}^{0} \pi^{0} \gamma$ final states originating from $B^{+} B^{-}$decays. Numerical estimates are given for Monte Carlo data sets of $799 / \mathrm{fb}$. Only topologies appearing more than once are shown. (The remaining contribution is called 'other'.)

- the $B$ meson polar angle in the center of mass frame was not used in the fit since it is correlated with $m_{\mathrm{ES}}$ signal and $\Delta E$ for background and its signalbackground separation power was found to be negligible.

- for the $B^{0} \rightarrow K^{* 0} \gamma$ decays the $K^{* 0}$ invariant mass was used in the fit. Since the lineshape of the invariant mass of $K_{S} \pi^{0}$ system is not well known outside the $K^{* 0}$ resonance region, it was not used in the fit for $B^{0} \rightarrow K_{S}^{0} \pi^{0} \gamma$ decays.

In order to account for the $B \bar{B}$ background contribution, which is not negligible in the $B^{0} \rightarrow K_{S}^{0} \pi^{0} \gamma$ case, the full likelihood was modified with respect to that used 
in the $B^{0} \rightarrow K_{S}^{0} \pi^{0}$ analysis and a $B \bar{B}$ background component was added:

$$
\begin{aligned}
& \mathcal{L}=e^{-\left(N_{S}+N_{B}+N_{B \bar{B}}\right)} \prod_{i \in \text { good }}^{N_{\text {good }}}\left(N_{S} f_{\text {good }}^{S} \epsilon_{c_{i}}^{S} \quad \cdot \quad \mathcal{P}^{S}\left(m_{\mathrm{ES} i}\right) \mathcal{P}^{S}\left(\Delta E_{i}\right) \mathcal{P}^{S}\left(\mathcal{F}_{i, c}\right) P_{S}^{c}\left(\Delta t_{i}, T \mid \sigma_{\Delta t_{i}}\right)+\right. \\
& N_{B} f_{\text {good }}^{B} \epsilon_{c_{i}}^{B} \quad \cdot \quad \mathcal{P}^{B}\left(m_{\mathrm{ES} i}\right) \mathcal{P}^{B}\left(\Delta E_{i}\right) \mathcal{P}^{B}\left(\mathcal{F}_{i, c}\right) P_{B}^{c}\left(\Delta t_{i}, T \mid \sigma_{\Delta t_{i}}\right)+ \\
& \left.N_{B \bar{B}} f_{\text {good }}^{B \bar{B}} \epsilon_{c_{i}}^{B \bar{B}} \quad \cdot \quad \mathcal{P}^{B \bar{B}}\left(m_{\mathrm{ES} i}\right) \mathcal{P}^{B \bar{B}}\left(\Delta E_{i}\right) \mathcal{P}^{B \bar{B}}\left(\mathcal{F}_{i, c}\right) P_{B \bar{B}}^{c}\left(\Delta t_{i}, T \mid \sigma_{\Delta t_{i}}\right)\right) \times \\
& \prod_{i \in \text { bad }}^{N_{\text {bad }}}\left(N_{S}\left(1-f_{\text {good }}^{S}\right) \epsilon_{c_{i}}^{S} \cdot \mathcal{P}^{S}\left(m_{\mathrm{ES} i}\right) \mathcal{P}^{S}\left(\Delta E_{i}\right) \mathcal{P}^{S}\left(\mathcal{F}_{i}\right) \mathcal{P}_{c}^{S}(T)+\right. \\
& N_{B}\left(1-f_{\text {good }}^{B}\right) \epsilon_{c_{i}}^{B} \quad \cdot \quad \mathcal{P}^{B}\left(m_{\mathrm{ES} i}\right) \mathcal{P}^{B}\left(\Delta E_{i}\right) \mathcal{P}^{B}\left(\mathcal{F}_{i}\right) \mathcal{P}_{c}^{B}(T)+ \\
& N_{B \bar{B}}\left(1-f_{\text {good }}^{B \bar{B}}\right) \epsilon_{c_{i}}^{B \bar{B}} \quad \cdot \quad \mathcal{P}^{B \bar{B}}\left(m_{\mathrm{ES} i}\right) \mathcal{P}^{B \bar{B}}\left(\Delta E_{i}\right) \mathcal{P}^{B \bar{B}}\left(\mathcal{F}_{i}\right) \mathcal{P}_{c}^{B \bar{B}}\left(\left(\Delta t_{i}, T\right)\right)
\end{aligned}
$$

Figure 6.3 shows the s-Plots of signal, background and $B \bar{B}$ background in data for different PDFs used in the fit. These distributions show how each component of the total PDF looks like if the other two components are subtracted. Signal and $B \bar{B}$ background PDFs were parametrized using Monte Carlo simulations corrected for a discrepancy between data and Monte Carlo in the calorimeter energy measurements. The parameters of the continuum background PDFs were floated in the fit on data.

\subsection{Results}

In the fit to the $B^{0} \rightarrow K^{* 0} \gamma$ sample it was found that the signal yield is $157 \pm 16$ events. The $C P$ violating asymmetry parameters were found to be:

$$
\begin{aligned}
& S_{K^{* 0} \gamma}=-0.21 \pm 0.40(\text { stat }) \pm 0.05(\text { syst }) \\
& C_{K^{* 0} \gamma}=-0.40 \pm 0.23(\text { stat }) \pm 0.03(\text { syst }) .
\end{aligned}
$$



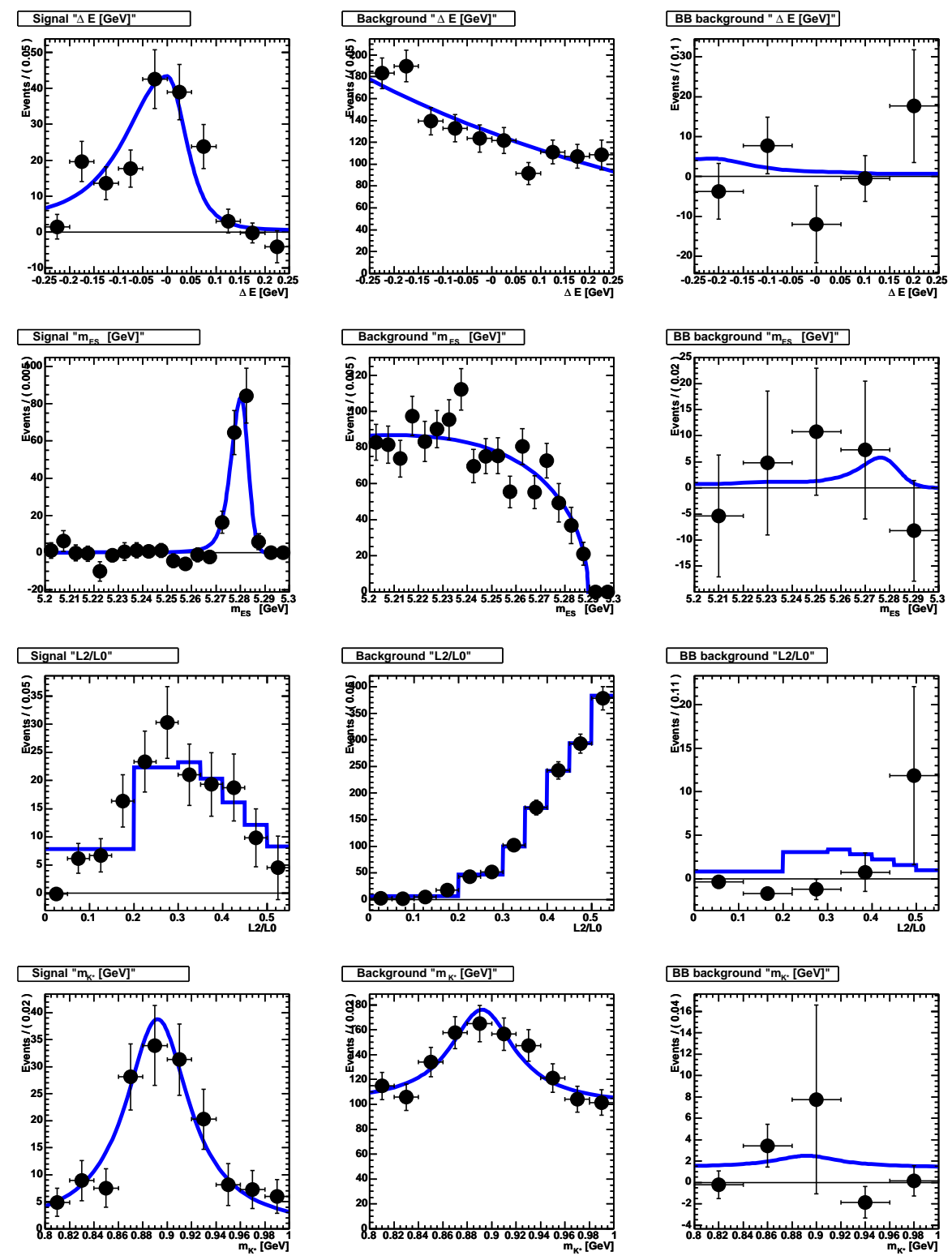

Figure 6.3: Results of a three component fit (signal, background and $B \bar{B}$ background) on data presented in the form of s-Plots. The blue curves are the fit model PDFs and the black dots correspond to data. The left set of plots is a set of signal distributions with subtracted continuum and $B \bar{B}$ background. The middle plots are background distributions and the right plots correspond to the $B \bar{B}$ background distributions. 
The value of $C_{K^{* 0 \gamma}}$ is consistent with the expectation of no direct $C P$ violation. Since its uncertainty is much larger than that obtained from the direct $C P$ violating asymmetry measurements in self-tagging decays [39], a fit with $C_{K^{* 0} \gamma}$ fixed to zero was also performed, yielding:

$$
S_{K^{* 0} \gamma}(C \equiv 0)=-0.22 \pm 0.42 \text { (stat) } \pm 0.05 \text { (syst). }
$$

The small increase in the error on $S_{K^{* 0} \gamma}(C \equiv 0)$ is a consequence of the asymmetry of the likelihood distribution. Figure 6.4 shows the likelihood contours in the $S-C$ plane with an apparent asymmetry.

Figure 6.5 shows the background subtracted $K_{S} \pi^{0}$ invariant mass distribution for $B^{0} \rightarrow K_{S}^{0} \pi^{0} \gamma$ candidates. The $K^{*}(892)$ resonance is clearly visible and there is some evidence for the $K_{2}^{*}(1430)$. Figure 6.6 shows the background subtracted distributions for $m_{\mathrm{ES}}$ and $\Delta E$ for events outside the $K^{*}(892)$ resonance region $\left(m_{K_{S} \pi^{0}} \in\right.$ $[1.1,1.8] \mathrm{GeV})$. In the fit to this sample, $59 \pm 13$ signal events were found and the asymmetry terms, $S$ and $C$, are found to be:

$$
\begin{gathered}
S_{K_{S} \pi^{0} \gamma}=0.9 \pm 1.0(\text { stat }) \pm 0.2(\text { syst }) \\
C_{K_{S} \pi^{0} \gamma}=-1.0 \pm 0.5(\text { stat }) \pm 0.2(\text { syst }) .
\end{gathered}
$$




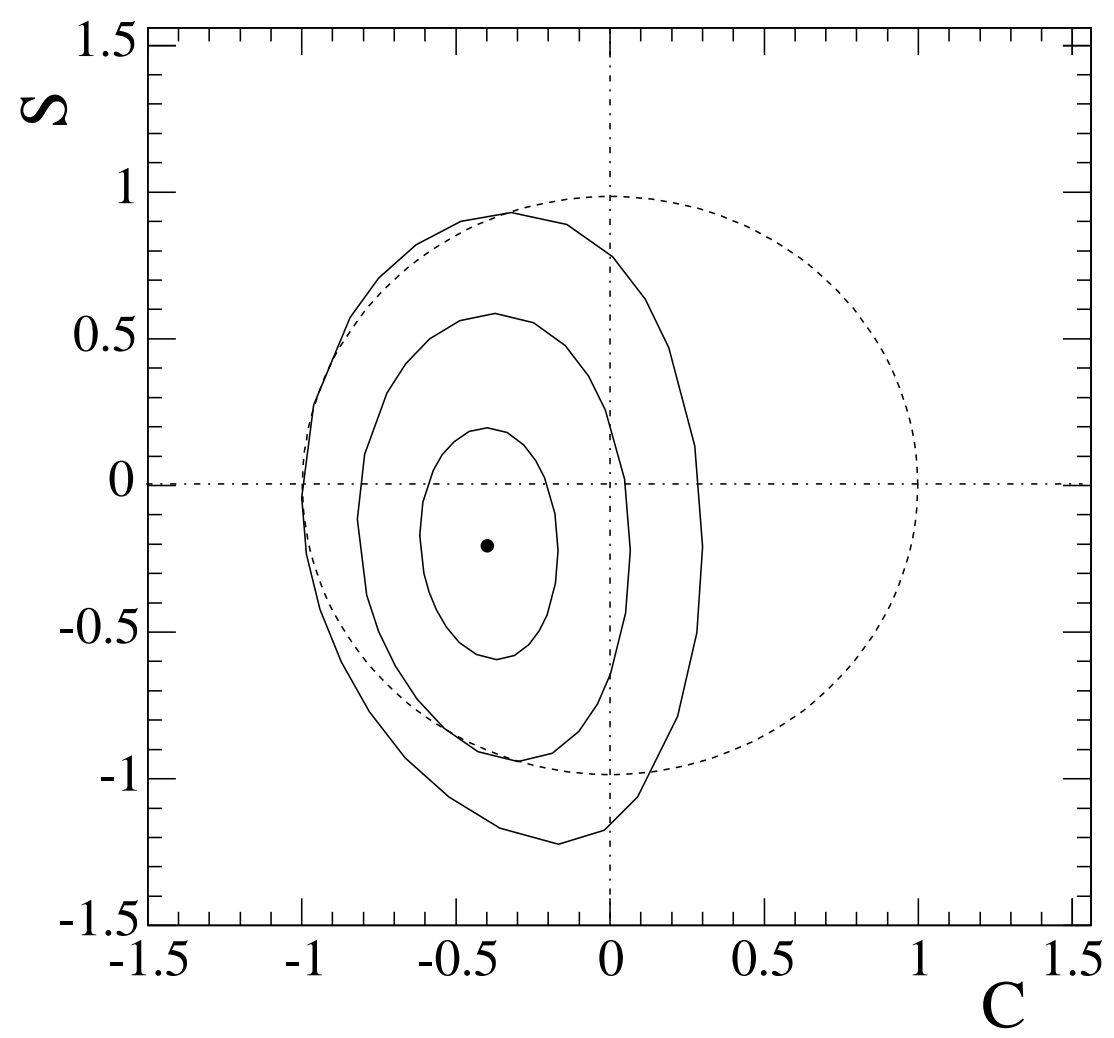

Figure 6.4: Constant-likelihood contours in the $S-C$ plane for $B^{0} \rightarrow K^{* 0} \gamma$ corresponding to 1,2 and $3 \sigma$ change in the likelihood. The dashed circle is the physical boundary.

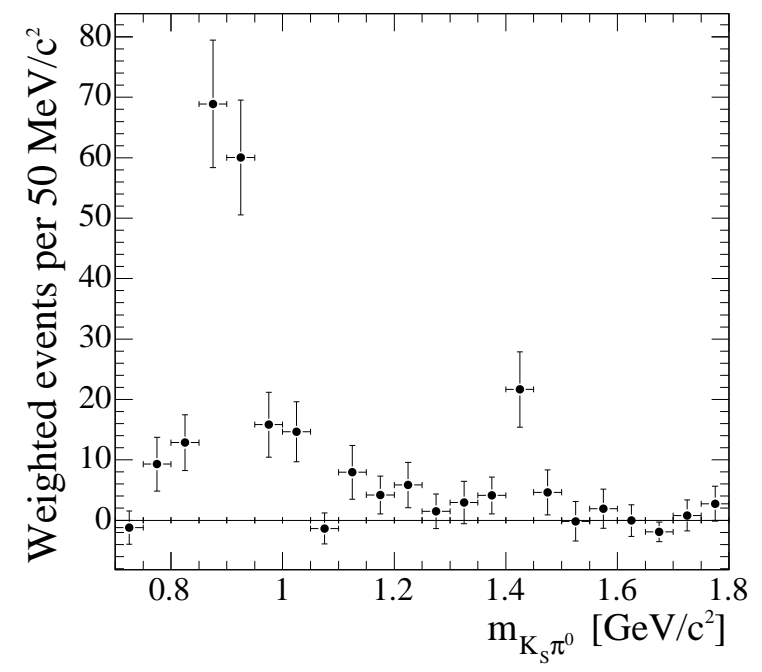

Figure 6.5: Background subtracted distribution for the $K_{S} \pi^{0}$ invariant mass obtained with the s-Plot technique. The cut on the $K^{*}$ helicity angle was not applied. 

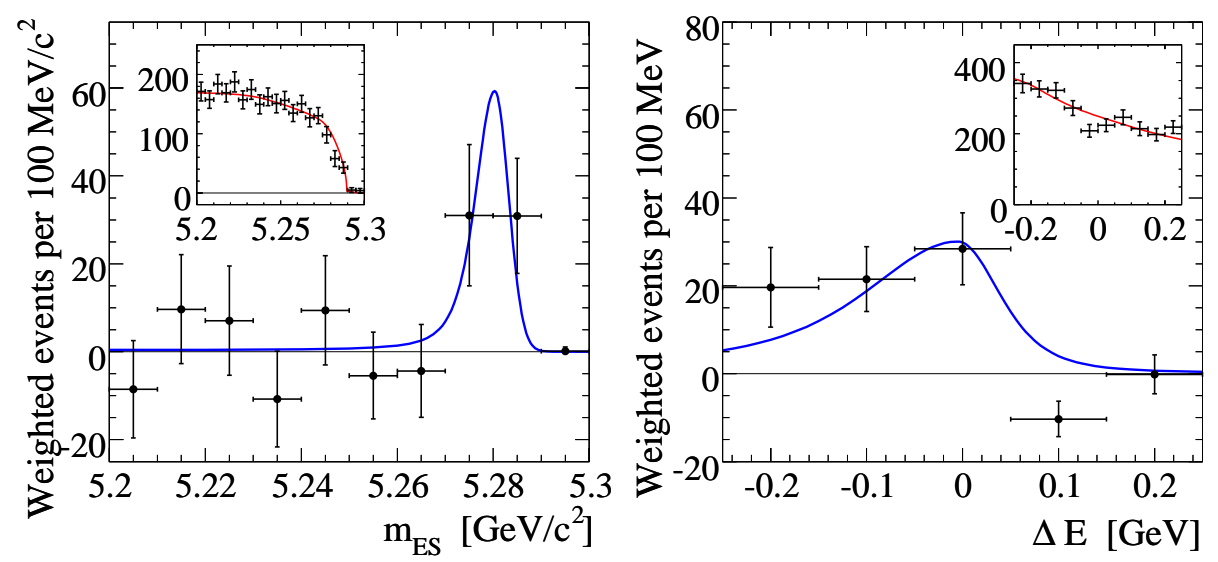

Figure 6.6: Signal and background (inset) distributions for $m_{\mathrm{ES}}$ (left) and $\Delta E$ (right) for $B^{0} \rightarrow K_{S}^{0} \pi^{0} \gamma$ candidates with $m_{K_{S} \pi^{0}} \in[1.1,1.8] \mathrm{GeV}$ obtained the s-Plot technique. The curves represent the PDFs used in the fit, normalized to the fitted yield.

\subsection{Validations and systematic effects evaluation}

Evaluation of the systematic errors and the fit validation are similar to that of the $B^{0} \rightarrow K_{S}^{0} \pi^{0}$ analysis, but there are significant differences coming from the fact that the $B \bar{B}$ background is a significant effect especially in the case of non- $K^{*} B^{0} \rightarrow$ $K_{S}^{0} \pi^{0} \gamma$. Introduction of the third component in the fit, which corresponds to the $B \bar{B}$ background, makes the fit much more complex and more tests are required to validate its performance. Another problem with the $B \bar{B}$ background lays in the fact that in most cases the $B \bar{B}$ background is hardly distinguishable from the continuum background. Underestimation or overestimation of the $B \bar{B}$ background yield might affect the asymmetry measurements, since in the $B \bar{B}$ background $C P$ violation can occur.

In order to properly measure the asymmetries in signal one has to know the relative fraction of $B \bar{B}$ background events and the $C P$ asymmetry of the $B \bar{B}$ background in 
the final data sample. A systematic underestimation of the $B \bar{B}$ background yield in the fit was found and traced to the imperfect knowledge of the $B \bar{B}$ background PDF. In the "toy" Monte Carlo experiments, when the same PDF is used for event generation and fitting, the bias in the $B \bar{B}$ background yield was found to be negligible, but as soon as some variation of the PDF was introduced, the underestimation of the $B \bar{B}$ background yield became significant.

In the case of large $B \bar{B}$ background, its yield, PDF and the asymmetries can be measured directly in data. With the current data sample the uncertainties in these measurements are too high and in order to estimate the systematic errors on the asymmetry measurements several $B \bar{B}$ background PDFs were used to compare with the nominal fit result, where a set of Monte Carlo $B \bar{B}$ background events was used. The $B \bar{B}$ yield extracted in the nominal fit was underestimated on average and for the systematic error estimations the expected $B \bar{B}$ yield from the Monte Carlo simulations was used. Several fits were performed using the extreme values of the $C P$ violating asymmetries in the $B \bar{B}$ background taking into account that the time-dependent $C P$ violation can only occur for a fraction of the $B \bar{B}$ background events. Events such as $b \rightarrow s \gamma$ are expected to have a negligible direct $C P$ violation asymmetry according to the $C P$ violation measurements in the semi-inclusive $B \rightarrow X_{S} \gamma$ decays. The time-dependent $C P$ violation can also only occur in the case of $B^{0} \bar{B}^{0}$ events and not $B^{+} B^{-}$

Table 6.4 summarizes the systematic uncertainties for the $B^{0} \rightarrow K^{* 0} \gamma$ and $B^{0} \rightarrow$ 


\begin{tabular}{l|cc|cc}
\hline \multirow{2}{*}{} & \multicolumn{2}{|c|}{$B^{0} \rightarrow K^{* 0} \gamma$} & \multicolumn{2}{c}{$B^{0} \rightarrow K_{S}^{0} \pi^{0} \gamma$} \\
\cline { 2 - 5 } & $\Delta S$ & $\Delta C$ & $\Delta S$ & $\Delta C$ \\
\hline beam-spot & 0.004 & 0.001 & 0.004 & 0.001 \\
SVT Alignment & 0.020 & 0.007 & 0.020 & 0.007 \\
data/MC resolution function & 0.014 & 0.004 & 0.014 & 0.004 \\
vertexing method & 0.018 & 0.013 & 0.018 & 0.013 \\
pdf parameterization & 0.016 & 0.010 & 0.035 & 0.078 \\
$B \bar{B}$ background & 0.031 & 0.018 & 0.141 & 0.141 \\
$\tau_{B}$ and $\Delta m_{d}$ & 0.005 & 0.005 & 0.005 & 0.005 \\
tag side interference & 0.003 & 0.014 & 0.003 & 0.014 \\
\hline Total: & $\mathbf{0 . 0 4 7}$ & $\mathbf{0 . 0 3 0}$ & $\mathbf{0 . 1 6 4}$ & $\mathbf{0 . 1 4 7}$ \\
\hline
\end{tabular}

Table 6.4: Systematic uncertainties on $S$ and $C$ for $B^{0} \rightarrow K^{* 0} \gamma$ and $B^{0} \rightarrow K_{S}^{0} \pi^{0} \gamma$ samples

$K_{S}^{0} \pi^{0} \gamma$ decays. Both for the $B^{0} \rightarrow K^{* 0} \gamma$ sample and for the non- $K^{* 0}$ sample, the uncertainty is dominated by the $B \bar{B}$ background. Other systematic effects are identical or similar to that of the $B^{0} \rightarrow K_{S}^{0} \pi^{0}$ analysis and have small impact on the total systematic error estimation.

\subsection{Summary}

In this chapter the result of the time-dependent $C P$ violating asymmetry measurement was presented for the $B^{0} \rightarrow K_{S}^{0} \pi^{0} \gamma$ decay for two $K_{S}^{0} \pi^{0}$ mass ranges: the $K^{*}$ decay with $m_{K_{S}^{0} \pi^{0}} \in[0.8,1.0] \mathrm{GeV}$ and non- $K^{*}$ decay with $m_{K_{S}^{0} \pi^{0}} \in[1.1,1.8] \mathrm{GeV}$. It was 
found that

$$
\begin{aligned}
S_{K^{* 0} \gamma} & =-0.21 \pm 0.40(\text { stat }) \pm 0.05(\text { syst }) \\
C_{K^{* 0} \gamma} & =-0.40 \pm 0.23(\text { stat }) \pm 0.03(\text { syst }) \\
S_{K_{S} \pi^{0} \gamma} & =0.9 \pm 1.0(\text { stat }) \pm 0.2(\text { syst }) \\
C_{K_{S} \pi^{0} \gamma} & =-1.0 \pm 0.5(\text { stat }) \pm 0.2(\text { syst }) .
\end{aligned}
$$

These results are statistics limited and the systematic uncertainties will improve with higher statistics. 


\section{Chapter 7}

\section{Conclusions}

In this dissertation I have presented measurements of the time-dependent $C P$ violating asymmetries in the decays $B^{0} \rightarrow K_{S}^{0} \pi^{0}$ and $B^{0} \rightarrow K_{S}^{0} \pi^{0} \gamma$. In the case of the $B^{0} \rightarrow$ $K_{S}^{0} \pi^{0}$ the Standard Model predicts the $S$ term of the $C P$ violating asymmetry to be:

$$
S_{K_{S}^{0} \pi^{0}}^{\mathrm{SM}} \approx \sin 2 \beta
$$

with a theoretical uncertainty in $|\Delta S|$ of around 0.1 , where $|\Delta S|$ is the Standard Model prediction on the deviation of the $S$ term in the $B^{0} \rightarrow K_{S}^{0} \pi^{0}$ decays from that of $b \rightarrow c \bar{c} s$ decays. $\sin 2 \beta$ has been measured in the $b \rightarrow c \bar{c} s$ decays to be 
$0.69 \pm 0.03[39]$. In this work we have found:

$$
\begin{aligned}
S_{K_{S}^{0} \pi^{0}} & =0.35_{-0.33}^{+0.30}(\text { stat }) \pm 0.04(\text { syst }) \\
C_{K_{S}^{0} \pi^{0}} & =0.06 \pm 0.18(\text { stat }) \pm 0.03(\text { syst }) \\
\mathcal{B}\left(B^{0} \rightarrow K_{S}^{0} \pi^{0}\right) & =(11.4 \pm 0.9(\text { stat }) \pm 0.6(\text { syst })) \times 10^{-6} .
\end{aligned}
$$

These results are compatible with the Standard Model prediction.

The Belle collaboration followed the original measurement by the BABAR collaboration and their most recent result [41] of time-dependent $C P$ asymmetries based on 386 million $B \bar{B}$ pairs is

$$
\begin{aligned}
& S_{K_{S}^{0} \pi^{0}}=0.22 \pm 0.47(\text { stat }) \pm 0.08(\text { syst }) \\
& C_{K_{S}^{0} \pi^{0}}=-0.11 \pm 0.18(\text { stat }) \pm 0.08(\text { syst })
\end{aligned}
$$

The current world average is $S_{K_{S}^{0} \pi^{0}}=0.31 \pm 0.26$. The difference in the accuracy of the measurements can be attributed to the significant advantage of the $B A B A R$ detector over the earlier version of the Belle silicon vertex tracker for decays with the vertex reconstruction technique used in these analyses. ${ }^{1}$ It is expected that with a

\footnotetext{
${ }^{1}$ The $B A B A R$ vertex detector has bigger size and larger number of layers than the Belle vertex detector. The relative fraction of "good" events used for $\Delta t$ measurement is defined by the fraction of the $K_{S}^{0}$ mesons, which decay inside the vertex detector. Significant part of the current data sample used by the Belle collaboration is collected with the old vertex detector, which had only 3 layers and small size (outer layer radius was $60.5 \mathrm{~mm}$ ). The SVT of the BABAR detector has 5 layers with the outer layer radius of $144 \mathrm{~mm}$. The new vertex detector installed by Belle collaboration in September 2003 has four layers with a slightly bigger outer layer radius $-88 \mathrm{~mm}$.
} 
data sample of about $2 \mathrm{ab}^{-1}$ the experimental error on the $S_{K_{S}^{0} \pi^{0}}$ can be reduced to 0.10 with systematic error at the level of 0.01 .

The average asymmetry in the $b \rightarrow q \bar{q} s$ charmless decays indicate some discrepancy with the average asymmetry in the $b \rightarrow c \bar{c} s$ decays, which contradicts the Standard Model prediction. The recent update of the world average numbers [39] is summarized in Figure 7.1. Neglecting the theoretical uncertainties on the $S$ term in $b \rightarrow q \bar{q} s$, the discrepancy is at the level of 2.8 "sigma". Table 7.1 summarizes the theoretical predictions of the difference in the $S$ term between $b \rightarrow q \bar{q} s$ and $b \rightarrow c \bar{c} s$ decays using the QCD factorization model [21].

In the Standard Model the $S$ term of the time-dependent $C P$ asymmetry (equation 2.14) for decay modes resulting from the process $b \rightarrow s \bar{s} s$ is equal to $-\eta_{C P} \sin 2 \beta$, where $\eta_{C P}$ is the $C P$ eigen-value of the final state. This follows from the fact that such decays can only proceed via a single penguin diagram with zero weak phase. Final states such as $\phi K_{S}^{0}, \eta^{\prime} K_{S}^{0}, K_{S}^{0} K_{S}^{0} K_{S}^{0}$ and $K^{+} K^{-} K_{S}^{0}$ are essentially products of $b \rightarrow s \bar{s} s$ decays with very small contribution of the $b \rightarrow u \bar{u} s$ decay amplitude. These decay modes are amongst the most theoretically clean channels for probing new physics in $b \rightarrow s$ loop processes. The $B^{0} \rightarrow K_{S}^{0} \pi^{0}$ decay originates from the $b \rightarrow d \bar{d} s$ diagram, which can only proceed via the penguin decay amplitude, but since $d \bar{d} s$ mixes strongly with $u \bar{u} s$, it is not possible to distinguish them. This allows for the presence of a tree decay amplitude with non-zero weak phase, which is color and doubly Cabibbo suppressed. 


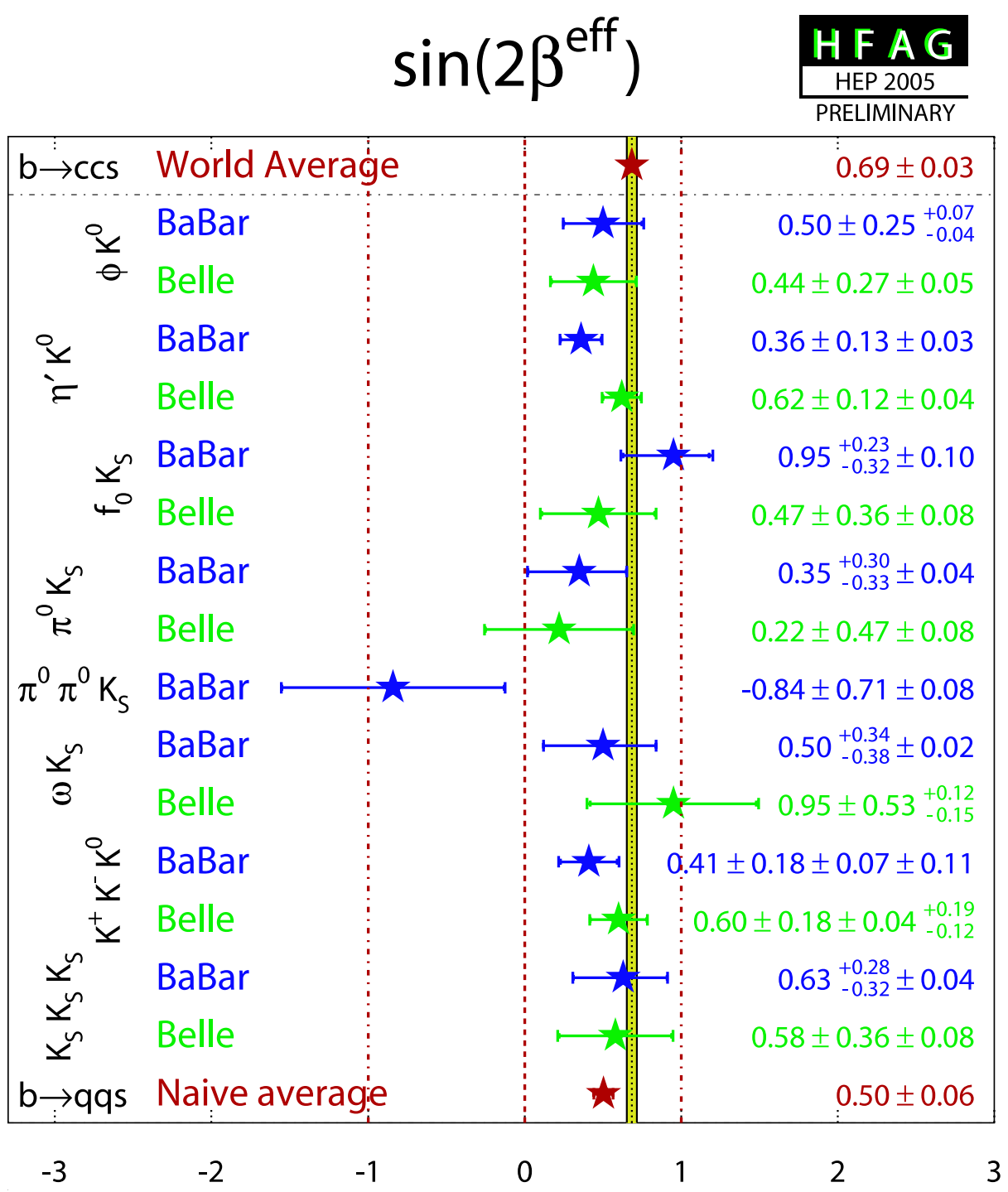

Figure 7.1: Experimental results for $-\eta_{f} S \approx \sin 2 \beta$ Summer 2005. The average numbers neglect the theoretical uncertainties. 


\begin{tabular}{l|rr} 
Mode & $\Delta S_{f}$ (Theory) & $\Delta S_{f}[$ Range $]$ \\
\hline$\pi^{0} K_{S}$ & $0.07_{-0.04}^{+0.05}$ & {$[+0.02,0.15]$} \\
$\rho^{0} K_{S}$ & $-0.08_{-0.12}^{+0.08}$ & {$[-0.29,0.02]$} \\
$\eta^{\prime} K_{S}$ & $0.01_{-0.01}^{+0.01}$ & {$[+0.00,0.03]$} \\
$\eta K_{S}$ & $0.10_{-0.07}^{+0.11}$ & {$[-1.67,0.27]$} \\
$\phi K_{S}$ & $0.02_{-0.01}^{+0.01}$ & {$[+0.01,0.05]$} \\
$\omega K_{S}$ & $0.13_{-0.08}^{+0.08}$ & {$[+0.01,0.21]$}
\end{tabular}

Table 7.1: The Standard Model expectation for $\Delta S_{f} \equiv-\eta_{f} S_{f}-\sin 2 \beta$ in the QCD factorization model.

In the Standard Model no significant $C P$ violation is expected in the $B^{0} \rightarrow K_{S}^{0} \pi^{0} \gamma$ decay:

$$
S_{K^{*} \gamma}^{\mathrm{SM}} \approx-0.04
$$

with the theoretical error of the order of 0.1 . The experimental measurement is consistent with this prediction:

$$
\begin{aligned}
S_{K^{* 0} \gamma} & =-0.21 \pm 0.40(\text { stat }) \pm 0.05 \text { (syst) } \\
C_{K^{* 0} \gamma} & =-0.40 \pm 0.23(\text { stat }) \pm 0.03(\text { syst }) \\
S_{K_{S} \pi^{0} \gamma} & =0.9 \pm 1.0(\text { stat }) \pm 0.2(\text { syst }) \\
C_{K_{S} \pi^{0} \gamma} & =-1.0 \pm 0.5(\text { stat }) \pm 0.2(\text { syst })
\end{aligned}
$$

Figure 7.2 shows the expected error on the $S$ term for the $B^{0} \rightarrow K^{* 0} \gamma$ decay as a function of the data sample size. It is expected that for a data sample of about $2 \mathrm{ab}^{-1}$, the uncertainty in the $S$ term measurement should be about 0.13 . 


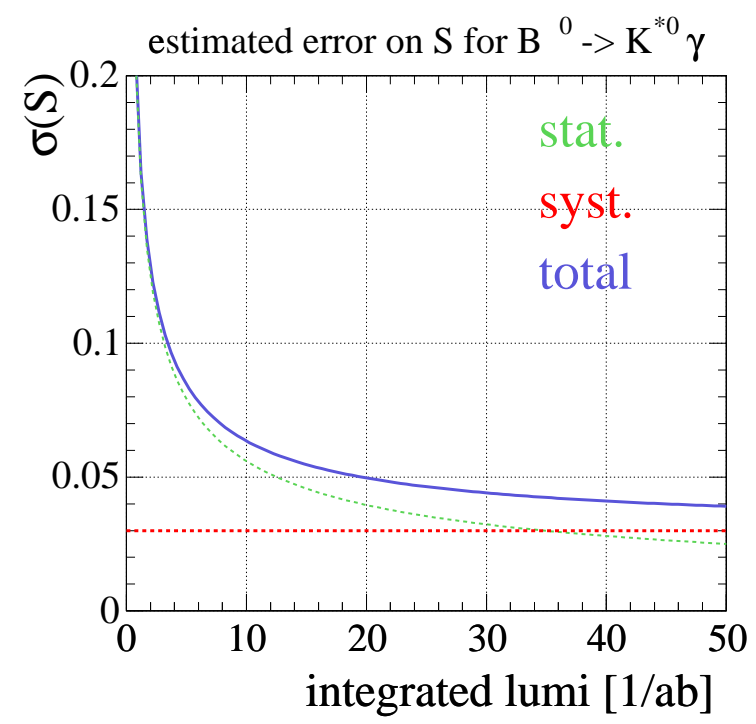

Figure 7.2: Distribution of the expected uncertainty on the $S$ term of time-dependent $C P$ violating asymmetry measurement for the $B^{0} \rightarrow K^{* 0} \gamma$ decay as a function of the dataset size.

All results presented in this dissertation are statistic limited. The allowed regions of the time-dependent asymmetry measurements correspond to substantial parts of the physically allowed regions. A significant increase in the data sample size is required to reach the theoretical uncertainty limits, which can happen by the end of BABAR data taking in 2008. 


\section{Bibliography}

[1] J. S. Schwinger, 'The Theory Of Quantized Fields. I", Phys. Rev. 82, 914 (1951); G. Lueders, 'Proof of the TCP theorem", Annals Phys. 2, 1 (1957) [Annals Phys. 281, 1004 (2000)]; W. Pauli, in 'Niels Bohr and the Development of Physics', edited by W. Pauli (McGraw-Hill, New York, 1955), p. 30; G. Lueders and B. Zumino, Phys. Rev. 106, 385 (1957).

[2] L. Wolfenstein, 'Parametrization Of The Kobayashi-Maskawa Matrix", Phys. Rev. Lett. 51, 1945 (1983).

[3] J. Charles et al. [CKMfitter Group], 'CP violation and the CKM matrix: Assessing the impact of the asymmetric B factories", Eur. Phys. J. C 41, 1 (2005) [arXiv:hep-ph/0406184]

[4] B. Aubert et al. [BABAR Collaboration], 'Search for T and CP violation in B0 - anti-B0 mixing with inclusive dilepton events", Phys. Rev. Lett. 88, 231801 (2002) [arXiv:hep-ex/0202041] 
[5] P. F. Harrison and H. R. Quinn [BABAR Collaboration], 'The BaBar physics book: Physics at an asymmetric B factory", SLAC-R-0504

[6] A. Alavi-Harati et al. [KTeV Collaboration], 'Observation of direct CP violation in $\mathrm{K}(\mathrm{S}, \mathrm{L}) \rightarrow$ pi pi decays", Phys. Rev. Lett. 83, 22 (1999); V. Fanti et al. [NA48 Collaboration], 'A new measurement of direct $\mathrm{CP}$ violation in two pion decays of the neutral kaon", Phys. Lett. B 465, 335 (1999); G. D. Barr et al. [NA31 Collaboration], 'A New measurement of direct CP violation in the neutral kaon system". Phys. Lett. B 317, 233 (1993).

[7] B. Aubert et al. [BaBar Collaboration], 'Observation of direct CP violation in B0 $\rightarrow$ K+ pi- decays", Phys. Rev. Lett. 93, 131801 (2004) [arXiv:hep-ex/0407057].

[8] H. Boos, T. Mannel and J. Reuter, 'The gold-plated mode revisited: sin(2beta) and B0 $\rightarrow \mathrm{J} /$ psi K(S) in the standard model", Phys. Rev. D 70, 036006 (2004) [arXiv:hep-ph/0403085].

[9] M. Schmaltz, 'Physics beyond the standard model (Theory): Introducing the little Higgs", Nucl. Phys. Proc. Suppl. 117, 40 (2003) [arXiv:hep-ph/0210415].

[10] 'Phenomenology and Lattice QCD", Proceedings of the Uehling Summer School on Phenomenology and Lattice QCD, eds. G. Kilcup and S. Sharpe, World Scientific (1995)

[11] J. Donoghue, E. Golowich and B. R. Holstein 'Dynamics of the StandardModel", Cambridge University Press (1992); G. Buchalla, A. J. Buras and M. E. Lauten- 
bacher, 'Weak Decays Beyond Leading Logarithms", Rev. Mod. Phys. 68, 1125 (1996) [arXiv:hep-ph/9512380]

[12] M. Neubert, 'Heavy quark symmetry", Phys. Rept. 245, 259 (1994) [arXiv:hep$\mathrm{ph} / 9306320]$

[13] J. Gasser and H. Leutwyler, 'Quark Masses", Phys. Rept. 87, 77 (1982)

[14] J. D. Bjorken, 'Topics In B Physics", Nucl. Phys. Proc. Suppl. 11, 325 (1989).

[15] M. Beneke, G. Buchalla, M. Neubert and C. T. Sachrajda, 'QCD factorization for exclusive, non-leptonic B meson decays: General arguments and the case of heavy-light final states", Nucl. Phys. B 591, 313 (2000) [arXiv:hep-ph/0006124].

[16] T. Hurth, 'Present status of inclusive rare B decays", Rev. Mod. Phys. 75, 1159 (2003) [arXiv:hep-ph/0212304].

[17] M. Gronau, Y. Grossman and J. L. Rosner, 'Interpreting the time-dependent CP asymmetry in B0 $\rightarrow$ pi0 K(S)," Phys. Lett. B 579, 331 (2004) [arXiv:hep$\mathrm{ph} / 0310020]$.

[18] M. Gronau, O. F. Hernandez, D. London and J. L. Rosner, 'Decays of B mesons to two light pseudoscalars", Phys. Rev. D 50, 4529 (1994) [arXiv:hep-ph/9404283].

[19] B. Aubert et al. [BABAR Collaboration], 'Measurements of branching fractions and $\mathrm{CP}$-violating asymmetries in $\mathrm{B} 0 \rightarrow \mathrm{pi}+$ pi-, $\mathrm{K}+$ pi-, $\mathrm{K}+\mathrm{K}$ - decays. $((\mathrm{B})) "$, Phys. Rev. Lett. 89, 281802 (2002) [arXiv:hep-ex/0207055]. 
[20] B. Aubert et al. [BABAR Collaboration], 'Improved measurements of branching fractions for $\mathrm{B} 0 \rightarrow$ pi + pi-, $\mathrm{K}+$ pi-, and search for $\mathrm{K}+\mathrm{K}-$ at BaBar", arXiv:hepex/0508046.

[21] M. Beneke, 'Corrections to sin(2beta) from CP asymmetries in $\mathrm{B} 0 \rightarrow$ (pi0, rho0, eta, eta', omega, Phi) K(S) decays", Phys. Lett. B 620, 143 (2005) [arXiv:hep$\mathrm{ph} / 0505075]$.

[22] D. Atwood, M. Gronau and A. Soni, "Mixing-induced CP asymmetries in radiative B decays in and beyond the standard model", Phys. Rev. Lett. 79, 185 (1997) [arXiv:hep-ph/9704272].

[23] B. Grinstein, Y. Grossman, Z. Ligeti and D. Pirjol, "The photon polarization in $\mathrm{B} \rightarrow \mathrm{X}$ gamma in the standard model", [arXiv:hep-ph/0412019].

[24] C. W. Bauer, S. Fleming, D. Pirjol and I. W. Stewart, 'An effective field theory for collinear and soft gluons: Heavy to light decays", Phys. Rev. D 63, 114020 (2001) [arXiv:hep-ph/0011336]

[25] T. Becher, R. J. Hill and M. Neubert, 'Factorization in B $\rightarrow$ V gamma decays", [arXiv:hep-ph/0503263]

[26] Y. Grossman and D. Pirjol, 'Extracting and using photon polarization information in radiative B decays", JHEP 0006, 029 (2000) [arXiv:hep-ph/0005069]. 
[27] M. Gronau, Y. Grossman, D. Pirjol and A. Ryd, 'Measuring the photon helicity in radiative B decays", Phys. Rev. Lett. 88, 051802 (2002) [arXiv:hep-ph/0107254].

[28] D. Boutigny et al. [BABAR Collaboration], "BaBar technical design report", SLAC-R-0457

[29] B. Aubert et al. [BABAR Collaboration], "The BaBar detector", Nucl. Instrum. Meth. A 479, 1 (2002) [arXiv:hep-ex/0105044]

[30] http://roofit.sourceforge.net/

[31] D. H. Wright et al. [BABAR Computing Group], 'Using Geant4 in the BaBar simulation", eConf C0303241, TUMT006 (2003) [arXiv:hep-ph/0305240].

[32] B. Aubert et al. [BABAR Collaboration], 'Measurements of the $\mathrm{B} \rightarrow \mathrm{X} / \mathrm{s}$ gamma branching fraction and photon spectrum from a sum of exclusive final states", arXiv:hep-ex/0508004.

[33] J. Alexander et al. [Heavy Flavor Averaging Group], arXiv:hep-ex/0412073. Average computed from S. Nishida et al. [Belle Collaboration] Phys. Rev. Lett. 89, 231801 (2002) and B. Aubert et al. [BABAR Collaboration], Phys. Rev. D 70, 091105 (2004).

[34] H. S. Green, "A Generalized Method of Field Quantization", Phys. Rev. vol.902 (1953); H. C. von Baeyer, "Kaon Decays and Pion Statistics", Phys. Rev. 
135, B189-B190 (1964); S. A. Bludman, " $K 2 \rightarrow \pi^{+} \pi^{-}$and the Question of Bose Statistics for Pions", Phys. Rev. 138, B213-B215 (1965)

[35] M. Pivk and F. R. Le Diberder, 'sPlot: a statistical tool to unfold data distributions", arXiv:physics/0402083, Accepted for publication in Nucl. Instr. Methods

[36] S. Eidelman et al. [Particle Data Group], 'Review of particle physics", Phys. Lett. B 592, 1 (2004)

[37] G. Taylor [Belle Collaboration], 'The Belle Silicon Vertex Detector: Present performance and upgrade plans", Nucl. Instrum. Meth. A 501, 22 (2003)

[38] David Atwood, Tim Gershon, Masashi Hazumi, Amarjit Soni, "Mixing-Induced CP Violation in $B \rightarrow P_{1} P_{2} \gamma$ in Search of Clean New Physics Signals", [arXiv:hep$\mathrm{ph} / 0410036]$

[39] Heavy Flavor Averaging Group, http://www.slac.stanford.edu/xorg/hfag/, arXiv:hep-ex/0412073, arXiv:hep-ex/0505100

[40] A. L. Kagan and M. Neubert, "QCD anatomy of B $\rightarrow$ X/s gamma decays", Eur. Phys. J. C 7, 5 (1999), [arXiv:hep-ph/9805303]

[41] K. Abe et al. [Belle Collaboration], 'Time-dependent CP asymmetries in $\mathrm{b} \rightarrow \mathrm{s}$ anti-q q transitions and $\sin (2 \mathrm{phi}(1))$ in $\mathrm{B} 0 \rightarrow \mathrm{J} / \mathrm{psi}$ K0 decays with 386 million B anti-B peairs", arXiv:hep-ex/0507037. 